TUM-HEP-864/12

TTK-12-44

SFB/CPP-12-77

UWThPh-2012-31

IFIC/12-70

27 October 2012

\title{
Non-relativistic pair annihilation of nearly mass degenerate neutralinos and charginos I. General framework and S-wave annihilation
}

\author{
M. Beneke ${ }^{a, b}$, C. Hellmann ${ }^{a, b}$ and P. Ruiz-Femenía ${ }^{c, d}$ \\ ${ }^{a}$ Physik Department T31, \\ James-Franck-Straße, Technische Universität München, \\ D-85748 Garching, Germany \\ ${ }^{b}$ Institut für Theoretische Teilchenphysik und Kosmologie, \\ RWTH Aachen University, D-52056 Aachen, Germany \\ ${ }^{c}$ University of Vienna - Faculty of Physics \\ Boltzmanngasse 5, A-1090 Wien, Austria \\ ${ }^{d}$ Instituto de Física Corpuscular (IFIC), CSIC-Universitat de València \\ Apdo. Correos 22085, E-46071 Valencia, Spain
}

\begin{abstract}
We compute analytically the tree-level annihilation rates of a collection of nonrelativistic neutralino and chargino two-particle states in the general MSSM, including the previously unknown off-diagonal rates. The results are prerequisites to the calculation of the Sommerfeld enhancement in the MSSM, which will be presented in subsequent work. They can also be used to obtain concise analytic expressions for MSSM dark matter pair annihilation in the present Universe for a large number of exclusive two-particle final states.
\end{abstract}




\section{Introduction}

The presence of dark matter (DM) in the Universe is one of the few empirical evidences that the current Standard Model of particle physics cannot be complete. The dark matter density $\Omega_{\mathrm{DM}}=0.111(6) / h^{2}(h=0.710(25))$ [1] is now determined very precisely from various observations. While the particle nature and genesis of the dark matter relic density remains unknown, it is intriguing that it can be explained naturally through thermal production and freeze-out of a particle with electroweak interaction strengths and mass of order of the $\mathrm{TeV}$ scale. In this simple scenario freeze-out occurs when the Universe cools below the mass of the particle and the DM particles become nonrelativistic, with typical velocities of order $v \sim 0.2 c$. The DM pair-annihilation cross section, which determines the relic density, can then be expanded in a Taylor series in $v$, and keeping only the first two terms is usually a very good approximation:

$$
\sigma_{\mathrm{ann}} v_{\mathrm{rel}} \approx a+b v_{\mathrm{rel}}^{2}
$$

Here $v_{\text {rel }}=\left|\vec{v}_{1}-\vec{v}_{2}\right|$ denotes the relative velocity of the annihilating particles in their center-of-mass frame. Furthermore, when dark matter particles pair-annihilate in the present Universe, potentially revealing themselves in cosmic ray signatures, the typical velocities are $v \sim 10^{-3} c$, and the annihilation occurs even deeper in the non-relativistic regime.

Among the many models that contain weakly interacting dark matter candidates at the TeV scale, the minimal supersymmetric standard model (MSSM) has been studied most extensively, and for quite some time [2,3]. Several programs are available [4,5] which compute the annihilation cross section of the lightest neutralino, together with possible co-annihilation processes, in the MSSM numerically in the tree-level approximation. The observed relic density then provides a valuable constraint on the parameter space of the model, complementary to those from collider physics. Given the precision of $\Omega_{\mathrm{DM}}$, it seems desirable to compute the cross section parameters $a, b$ at the one-loop level. This, however, is a daunting task due to the complexity of complete one-loop calculations in the MSSM, and the number of individual annihilation processes that add up to the total cross section. Nevertheless, such calculations have been performed for certain scenarios where QCD corrections are the most important ones [6] 8], or in certain approximations [9, 10]. The calculation of the full electroweak corrections has been started [11,12,

There exist situations when quantum corrections become exceedingly large and cannot be neglected. In non-relativistic scattering and annihilation of DM particles this happens when the Coulomb (Yukawa) force generated by massless (massive) particle exchange between the DM particles becomes strong at small relative velocities, a phenomenon also known as "Sommerfeld effect". In the MSSM this situation is naturally realized when the lightest neutralino (LSP) has mass above one $\mathrm{TeV}$, in which case the neutralino is almost a pure gauge eigenstate, and degeneracies and co-annihilation effects in the neutralino-chargino sector are generic. The Sommerfeld effect in the MSSM was first studied in the wino- and Higgsino-limit by Hisano et al. [13, 14, and subsequently in "minimal dark matter models" [15] that resemble the MSSM in the above-mentioned lim- 
its. In these heavy dark matter scenarios the annihilation cross section can be enhanced by more than an order of magnitude, since the typical distance of DM particles at small velocity is within the long-range part of the Yukawa potential generated by exchange of the electroweak $W$ and $Z$ gauge bosons. The suggestion [16] that the Sommerfeld enhancement due to the exchange of a new, light particle may generate an excess in the cosmic ray positron spectrum has generated a resurge of interest in this effect.

The present work aims at improving the calculation of the dark matter annihilation cross section and relic abundance by including the Sommerfeld radiative corrections in the general MSSM, beyond the previously considered wino- and Higgsino-limit. The idea is that even when the Sommerfeld correction is not of order one, unlike in scenarios of TeV scale LSPs, it may still constitute the dominant radiative correction in a significantly larger portion of the MSSM parameter space. Related work has been undertaken recently in [17 20]. Our approach differs from or extends these works in several aspects.

- We use the non-relativistic effective theory approach to separate the short-distance annihilation process from the long-distance Sommerfeld effect, which is encoded in the matrix elements of local four-fermion operators. The approach is very similar to the NRQCD treatment of quarkonium annihilation [21], except that we deal with scattering states of several species of particles interacting through the electroweak Yukawa force.

- Since electroweak gauge boson exchange may change the two-particle state (for instance, scatter a neutralino pair into a pair of oppositely charged charginos), the annihilation process is described by a matrix in the space of two-particle states, which is not diagonal. The off-diagonal terms cannot be obtained from the treelevel cross sections computed by numerical programs, and have not been considered previously, except in the simplified situation of the strict wino- and Higgsinolimit [13 15, 20].

- We compute the expansion of the short-distance annihilation cross section analytically rather than numerically. The only systematic previous analytic calculation [22] refers to the annihilation of two LSPs, but does not include co-annihilation channels and the above-mentioned off-diagonal annihilation matrix entries.

- The non-relativistic annihilation cross section can be organized in a partial wave expansion. The leading-order term $a$ in (1) contains the leading-order contributions from $S$-wave annihilations, whereas the second term $b$ encodes both $S$ - and $P$-wave annihilation contributions. The Sommerfeld correction factor is different for the $S$ - and $P$-wave contribution. Its consistent implementation therefore requires a separation of $b$ into its two components, which has not been done before, but can be implemented relatively easily within our analytic framework.

The present paper is devoted to the analytic calculation of the dominant $S$-wave annihilation coefficient $a$ and to outlining the general framework. The subleading term $b$ and 
the calculation of the Sommerfeld effect in the MSSM with almost degenerate neutralinos and charginos will be presented in [23] and [24], respectively.

The outline of this paper is as follows. In Sec. 2 we introduce the effective Lagrangian framework and the method of calculation. In the non-relativistic MSSM the short-distance annihilation process is encoded in the Wilson coefficient of a four-fermion operator. We introduce the required notation and discuss the expansion in the mass differences of the nearly degenerate neutralino and chargino species. Sec. 3 discusses various examples of annihilation cross sections obtained from our analytic calculation. In this section we also perform checks by comparing some diagonal annihilation matrix entries with numerical cross sections and with [22]. The complete analytic results are rather lengthy. We provide them in appendix A together with the rules to construct the MSSM coupling factors of the various diagrams. In Sec. 4 we explain why it is convenient to employ Feynman gauge despite the fact that this requires the computation of many unphysical final states. We also illustrate the importance of including the off-diagonal annihilation matrix entries in the computation of the Sommerfeld-corrected cross section on the example of a heavy wino-like MSSM parameter point. We summarize in Sec. 5 .

\section{Effective Lagrangian and method of calculation}

\subsection{The Lagrangian in the effective theory}

We describe the kinematics and interactions of neutralinos and charginos moving at small velocities within a non-relativistic effective theory (EFT), the non-relativistic MSSM (NRMSSM), that contains only nearly on-shell non-relativistic chargino and neutralino modes, while the effects from higher mass and virtual modes are encoded in the Wilson coefficients of higher-dimensional operators. The neutralinos and charginos described in the EFT approach are those whose masses are nearly degenerate with the mass $m_{\mathrm{LSP}}$ of the lightest neutralino. The corresponding effective Lagrangian is given by

$$
\mathcal{L}^{\mathrm{NRMSSM}}=\mathcal{L}_{\text {kin }}+\mathcal{L}_{\text {pot }}+\delta \mathcal{L}_{\text {ann }}+\text { higher order terms }
$$

The kinetic part of the Lagrangian for $n_{0} \leq 4$ non-relativistic neutralino species and $n_{+} \leq 2$ non-relativistic chargino species is given by

$$
\begin{aligned}
\mathcal{L}_{\text {kin }}= & \sum_{i=1}^{n_{0}} \xi_{i}^{\dagger}\left(i \partial_{t}-\left(m_{i}-m_{\mathrm{LSP}}\right)+\frac{\vec{\partial}^{2}}{2 m_{\mathrm{LSP}}}\right) \xi_{i} \\
& +\sum_{\psi=\eta, \zeta} \sum_{j=1}^{n_{+}} \psi_{j}^{\dagger}\left(i \partial_{t}-\left(m_{j}-m_{\mathrm{LSP}}\right)+\frac{\vec{\partial}^{2}}{2 m_{\mathrm{LSP}}}\right) \psi_{j} .
\end{aligned}
$$

The fields $\xi_{i}$ and $\psi_{j}=\eta_{j}, \zeta_{j}$ represent the non-relativistic two-component spinor fields

of non-relativistic neutralinos $\left(\chi_{i}^{0}\right)$ and charginos $\left(\chi_{j}^{-}\right.$and $\left.\chi_{j}^{+}\right)$, respectively. This EFT setup with one reference mass scale, $m_{\mathrm{LSP}}$, is suited for the description of (neutralino) 
dark matter annihilation processes in the present Universe as well as for the computation of dark matter co-annihilation reactions with further nearly mass-degenerate neutralinos and charginos in the context of the relic abundance calculation. The EFT framework can easily be extended to the case where the non-relativistic particle species are (nearly) mass-degenerate with respect to two distinct scales $m_{\mathrm{ref}}^{(1,2)}$, with $m_{\mathrm{ref}}^{(1)} \ll m_{\mathrm{ref}}^{(2)}$. In that case, the mass differences $\left(m_{k}-m_{\mathrm{LSP}}\right)$ in (3) have to be replaced by $m_{k}-m_{\text {ref, } k}$, where

each $m_{\text {ref, } k}$ is given by one of the scales $m_{\text {ref }}^{(1,2)}$. In that way, an entirety of hydrogen-like two-particle states can be described, within which a set of light, nearly mass-degenerate and another set of heavy, nearly mass-degenerate particles exists. Our results for the absorptive part of the Wilson coefficients, specified in Sec. 2.2 and given in the appendix, cover both the cases of a set of particles nearly mass-degenerate with the neutralino LSP and a set of non-relativistic hydrogen-like neutralino and chargino systems.

The term $\mathcal{L}_{\text {pot }}$ summarizes (instantaneous) Yukawa- and Coulomb potential interactions that arise through the exchange of SM gauge bosons and Higgs particles. The generic form of $\mathcal{L}_{\text {pot }}$ reads

$$
\mathcal{L}_{\text {pot }}=-\int d^{3} \vec{r} \Phi_{k l}^{\dagger}(x, \vec{r}) V_{i j k l}(r) \Phi_{i j}(x, \vec{r})
$$

where the fields $\Phi_{i j}$ describe a two-body state of the form $\chi_{i}^{0} \chi_{j}^{0}, \chi_{i}^{\mp} \chi_{j}^{ \pm}, \chi_{i}^{0} \chi_{j}^{ \pm}$or $\chi_{i}^{ \pm} \chi_{j}^{ \pm}$ and a sum over repeated indices is implicit. $V_{i j k l}(r)$ thus represents the potential interactions among two-body states $(i j)$ and $(k l)$ in configuration space, with $\vec{r}$ the spatial 3 -vector denoting the relative distance in the two-body system. The explicit form of the potentials between neutralino and chargino species for a given MSSM point will be given elsewhere [24].

\subsection{Basis of the dimension-6 operators in $\delta \mathcal{L}_{\text {ann }}$}

Within the NRMSSM, we aim to describe neutralino and chargino pair-annihilation processes into two-particle final states of Standard Model (SM) and (light) Higgs particles, which are not non-relativistic. The theory will contain effects from virtual and highermass Higgs and SUSY particle modes as well, encoded in the EFT operator-coefficients and parameters. The specific case of resonant $s$-channel pair-annihilation reactions can be covered by adding a resonance width in the analytic results that we give in the appendix. Yet we exclude the case of accidental mass degeneracies of further SUSY particles with the set of non-relativistic neutralinos and charginos.

The SM and light Higgs particle final states in the neutralino and chargino pairannihilation reactions are not described within the non-relativistic effective theory, as they are characterized by velocities outside the non-relativistic regime. However, since the hard inclusive pair-annihilation processes take place within distances of order $1 / m_{\mathrm{LSP}}$, we can incorporate the short-distance annihilation rates of non-relativistic neutralinos and charginos in the effective theory through the absorptive part of Wilson coefficients of local four-fermion operators in $\delta \mathcal{L}_{\text {ann }}$, following the approach of [21]. The full annihilation rates in the non-relativistic effective theory are given by the absorptive part of the matrix 


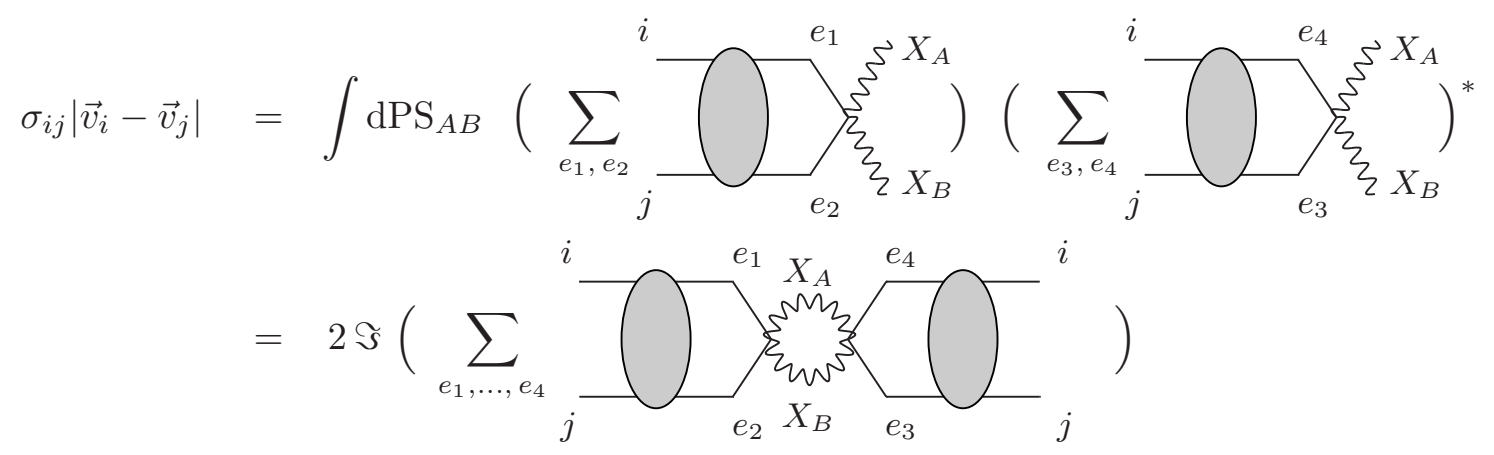

Figure 1: Diagrammatic picture for the relation among the annihilation amplitude and the absorptive part of the corresponding forward scattering amplitude in presence of long-range potential interactions.

elements of these four-fermion operators. While the matrix elements of the operators themselves may encode long-distance effects, giving rise to Sommerfeld enhancements, the contribution to the hard annihilation reaction factors out in the form of the Wilson coefficient.

In contrast to the application of this formalism to quarkonium annihilation in QCD [21], we are going to describe annihilations of scattering states instead of bound states and allow for more than one non-relativistic particle species. The latter allows for the possibility, that (long-range) potential interactions (indicated by the grey oval in Fig. 1) lead to transitions from the initially incoming two-particle state $\chi_{i} \chi_{j}$ to another two nearly on-shell non-relativistic two-particle state $\chi_{e_{1}} \chi_{e_{2}}$ prior to the annihilation reaction. Unitarity relates the phase space integrated product of annihilation amplitudes $\chi_{i} \chi_{j} \rightarrow$ $X_{A} X_{B}$ in the first line of Fig. 1 to the absorptive part of the forward scattering amplitude $\chi_{i} \chi_{j} \rightarrow \chi_{i} \chi_{j}$ depicted in the second line, where $X_{A} X_{B}$ generically denotes a pair of SM and light Higgs particles. Note that due to the presence of the long-range potential interactions, the hard annihilation reaction is determined by the absorptive part of the $\chi_{e_{1}} \chi_{e_{2}} \rightarrow \chi_{e_{4}} \chi_{e_{3}}$ amplitude, as can be seen in the second line of Fig. 1, It is worth to stress, that the $\chi_{e_{1}} \chi_{e_{2}}$ particle pair is not necessarily equal to the $\chi_{e_{4}} \chi_{e_{3}}$ pair, such that apart from true forward scattering reactions $\chi_{e_{1}} \chi_{e_{2}} \rightarrow \chi_{e_{1}} \chi_{e_{2}}$, we encounter off-diagonal $\chi_{e_{1}} \chi_{e_{2}} \rightarrow \chi_{e_{4}} \chi_{e_{3}}$ reactions as well.

In this paper we are concerned with the calculation of the absorptive part of $\chi_{e_{1}} \chi_{e_{2}} \rightarrow$ $X_{A} X_{B} \rightarrow \chi_{e_{4}} \chi_{e_{3}}$ 1-loop reactions, encoding the hard tree-level $\chi_{e_{1}} \chi_{e_{2}}$ and $\chi_{e_{4}} \chi_{e_{3}}$ annihilation processes. The full annihilation rates, including the long-range effects shall be studied elsewhere 24]. To determine the absorptive part of the Wilson coefficients, we evaluate the absorptive part of the hard $\chi_{e_{1}} \chi_{e_{2}} \rightarrow \chi_{e_{4}} \chi_{e_{3}}$ 1-loop scattering amplitude within the MSSM and match the result with the tree-level matrix element of four-fermion operators contained in $\delta \mathcal{L}_{\text {ann }}$ in the effective theory. At tree-level the annihilation rates can be given separately for every final state $X_{A} X_{B}$, since the tree-level processes are free from infrared divergences. In higher-orders the formalism applies to the inclusive 


\begin{tabular}{|c|c|c|}
\hline neutral reactions & single-charged reactions & double-charged reactions \\
\hline \hline$\chi^{0} \chi^{0} \rightarrow \chi^{0} \chi^{0}$ & $\chi^{0} \chi^{+} \rightarrow \chi^{0} \chi^{+}$ & $\chi^{+} \chi^{+} \rightarrow \chi^{+} \chi^{+}$ \\
$\chi^{0} \chi^{0} \rightarrow \chi^{-} \chi^{+}$ & $\chi^{-} \chi^{0} \rightarrow \chi^{-} \chi^{0}$ & $\chi^{-} \chi^{-} \rightarrow \chi^{-} \chi^{-}$ \\
$\chi^{-} \chi^{+} \rightarrow \chi^{0} \chi^{0}$ & & \\
$\chi^{-} \chi^{+} \rightarrow \chi^{-} \chi^{+}$ & & \\
\hline
\end{tabular}

Table 1: Collection of all $\chi_{e_{1}} \chi_{e_{2}} \rightarrow \chi_{e_{4}} \chi_{e_{3}}$ scattering reactions. The labels $e_{i}$ on the fields $\chi_{e_{i}}$ are suppressed in the above table. If $\chi_{e_{i}}$ represents a field $\chi_{e_{i}}^{0}$, the label $e_{i}$ can range over $e_{i}=1, \ldots, n_{0}$, whereas $e_{i}=1, \ldots, n_{+}$for the case of a $\chi_{e_{i}}^{ \pm}$field.

annihilation cross section [21], or to suitably defined infrared-safe final states.

The leading-order contributions in $\delta \mathcal{L}_{\text {ann }}$ are given by dimension- 6 four-fermion operators. For instance, the specific dimension- 6 four-fermion operator that encodes scattering of a non-relativistic incoming neutralino pair $\chi_{1}^{0} \chi_{1}^{0}$ in a ${ }^{1} S_{0}$ partial-wave state into an outgoing $\chi_{1}^{0} \chi_{1}^{0}$ state in the same ${ }^{1} S_{0}$ partial-wave configuration is given by

$$
\delta \mathcal{L}_{\text {ann }}^{d=6} \supset \frac{1}{4} f_{\{11\}\{11\}}^{\chi^{0} \chi^{0} \rightarrow \chi^{0} \chi^{0}}\left({ }^{1} S_{0}\right) \quad \xi_{1}^{\dagger} \xi_{1}^{c} \quad \xi_{1}^{c \dagger} \xi_{1}
$$

where the spinor $\xi^{c}$ is the charge conjugate of $\xi, \xi^{c}=-i \sigma^{2} \xi^{*}$, and $\sigma^{2}$ specifies the second Pauli matrix. Note that $\xi_{1}^{c \dagger} \xi_{1}$ represents the Lorentz invariant bilinear built from the non-relativistic particle field $\xi_{1}$, which destroys the incoming state of two identical $\chi_{1}^{0}$ particles. The factor $1 / 4$ denotes a normalization factor which compensates the symmetry factors arising from the number of identical contractions in the tree-level $\chi_{1}^{0} \chi_{1}^{0} \rightarrow \chi_{1}^{0} \chi_{1}^{0}$ matrix element. The symbol $f_{\{11\}\{11\}}^{\chi^{0} \chi^{0} \rightarrow \chi^{0} \chi^{0}}\left({ }^{1} S_{0}\right)$ denotes the Wilson coefficient corresponding to the dimension- 6 operator. We can generalize the above expression to include all possible spin-0 and spin-1 $S$-wave four-fermion operators at leading order in the non-relativistic expansion. Written in a compact form, the contribution of dimension6 operators in $\delta \mathcal{L}_{\text {ann }}$ reads

$$
\delta \mathcal{L}_{\mathrm{ann}}^{d=6}=\sum_{\chi \chi \rightarrow \chi \chi} \sum_{s=0,1} \frac{1}{4} f_{\left\{e_{1} e_{2}\right\}\left\{e_{4} e_{3}\right\}}^{\chi \chi \rightarrow \chi \chi}\left({ }^{2 s+1} S_{J}\right) \mathcal{O}_{\left\{e_{4} e_{3}\right\}\left\{e_{2} e_{1}\right\}}^{\chi \chi \rightarrow \chi \chi}\left({ }^{s+1} S_{J}\right)
$$

where $J=s$ for the case of $S$-wave operators considered here. The first sum, taken over all non-relativistic $2 \rightarrow 2$ neutralino and chargino scattering processes $\chi \chi \rightarrow \chi \chi$, implies the consideration of neutral scattering reactions as well as single-charged and doublecharged processes. The $\chi \chi \rightarrow \chi \chi$ reactions that we take into account are summarized in Tab. 1. The spin of the incoming and outgoing two-particle states can be either $s=0$ or $s=1$, such that the terms in the above Lagrangian $\delta \mathcal{L}_{\text {ann }}^{d=6}$ describe ${ }^{1} S_{0}$ and ${ }^{3} S_{1}$ partialwave scattering reactions. The $f_{\left\{e_{1} e_{2}\right\}\left\{e_{4} e_{3}\right\}}^{\chi \chi \rightarrow \chi \chi}\left({ }^{2 s+1} S_{J}\right)$ denote the Wilson coefficients, that correspond to the four-fermion operators $\mathcal{O}_{\left\{e_{4} e_{3}\right\}\left\{e_{2} e_{1}\right\}}^{\chi \chi \rightarrow \chi \chi}\left({ }^{2 s+1} S_{J}\right)$. The indices $e_{1}$ and $e_{2}\left(e_{3}\right.$ and $e_{4}$ ) refer to the neutralino or chargino species of the incoming (outgoing) particles, 


\begin{tabular}{|c|cc|cc|}
\hline$\chi_{e_{1}} \chi_{e_{2}} \rightarrow \chi_{e_{4}} \chi_{e_{3}}$ & $\mathcal{O}_{\left\{e_{4} e_{3}\right\}\left\{e_{2} e_{1}\right\}}^{\chi \chi \rightarrow}\left({ }^{1} S_{0}\right)$ & $\left.\mathcal{O}_{\left\{e_{4} e_{3}\right\}\left\{e_{2} e_{1}\right\}}^{\chi \chi \rightarrow \chi \chi}{ }^{3} S_{1}\right)$ \\
\hline \hline$\chi^{0} \chi^{0} \rightarrow \chi^{0} \chi^{0}$ & $\xi_{e_{4}}^{\dagger} \xi_{e_{3}}^{c}$ & $\xi_{e_{2}}^{c \dagger} \xi_{e_{1}}$ & $\xi_{e_{4}}^{\dagger} \vec{\sigma} \xi_{e_{3}}^{c}$ & $\xi_{e_{2}}^{c \dagger} \vec{\sigma} \xi_{e_{1}}$ \\
$\chi^{0} \chi^{0} \rightarrow \chi^{-} \chi^{+}$ & $\eta_{e_{4}}^{\dagger} \zeta_{e_{3}}^{c}$ & $\xi_{e_{2}}^{c \dagger} \xi_{e_{1}}$ & $\eta_{e_{4}}^{\dagger} \vec{\sigma} \zeta_{e_{3}}^{c}$ & $\xi_{e_{2}}^{c \dagger} \vec{\sigma} \xi_{e_{1}}$ \\
$\chi^{-} \chi^{+} \rightarrow \chi^{0} \chi^{0}$ & $\xi_{e_{4}}^{\dagger} \xi_{e_{3}}^{c}$ & $\zeta_{e_{2}}^{c \dagger} \eta_{e_{1}}$ & $\xi_{e_{4}}^{\dagger} \vec{\sigma} \xi_{e_{3}}^{c}$ & $\zeta_{e_{2}}^{c \dagger} \vec{\sigma} \eta_{e_{1}}$ \\
$\chi^{-} \chi^{+} \rightarrow \chi^{-} \chi^{+}$ & $\eta_{e_{4}}^{\dagger} \zeta_{e_{3}}^{c}$ & $\zeta_{e_{2}}^{c \dagger} \eta_{e_{1}}$ & $\eta_{e_{4}}^{\dagger} \vec{\sigma} \zeta_{e_{3}}^{c}$ & $\zeta_{e_{2}}^{c \dagger} \vec{\sigma} \eta_{e_{1}}$ \\
& & & \\
$\chi^{0} \chi^{+} \rightarrow \chi^{0} \chi^{+}$ & $\xi_{e_{4}}^{\dagger} \zeta_{e_{3}}^{c}$ & $\zeta_{e_{2}}^{c \dagger} \xi_{e_{1}}$ & $\xi_{e_{4}}^{\dagger} \vec{\sigma} \zeta_{e_{3}}^{c}$ & $\zeta_{e_{2}}^{c \dagger} \vec{\sigma} \xi_{e_{1}}$ \\
$\chi^{-} \chi^{0} \rightarrow \chi^{-} \chi^{0}$ & $\eta_{e_{4}}^{\dagger} \xi_{e_{3}}^{c} \xi_{e_{2}}^{c \dagger} \eta_{e_{1}}$ & $\eta_{e_{4}}^{\dagger} \vec{\sigma} \xi_{e_{3}}^{c}$ & $\xi_{e_{2}}^{c \dagger} \vec{\sigma} \eta_{e_{1}}$ \\
& & & \\
$\chi^{+} \chi^{+} \rightarrow \chi^{+} \chi^{+}$ & $\zeta_{e_{4}}^{\dagger} \zeta_{e_{3}}^{c}$ & $\zeta_{e_{2}}^{c \dagger} \zeta_{e_{1}}$ & $\zeta_{e_{4}}^{\dagger} \vec{\sigma} \zeta_{e_{3}}^{c}$ & $\zeta_{e_{2}}^{c \dagger} \vec{\sigma} \zeta_{e_{1}}$ \\
$\chi^{-} \chi^{-} \rightarrow \chi^{-} \chi^{-}$ & $\eta_{e_{4}}^{\dagger} \eta_{e_{3}}^{c}$ & $\eta_{e_{2}}^{c \dagger} \eta_{e_{1}}$ & $\eta_{e_{4}}^{\dagger} \vec{\sigma} \eta_{e_{3}}^{c}$ & $\eta_{e_{2}}^{c \dagger} \vec{\sigma} \eta_{e_{1}}$ \\
\hline
\end{tabular}

Table 2: Four-fermion operators for leading-order $S$-wave $\chi_{e_{1}} \chi_{e_{2}} \rightarrow \chi_{e_{4}} \chi_{e_{3}}$ transitions. The indices $e_{i}, i=1, \ldots, 4$ on the $\chi$-fields are suppressed in the first column. In addition to the specified operators there are redundant ones, which are obtained by interchanging the field-operator symbols $\xi, \eta$ or $\zeta$ (but not the labels) at the first and second and/or the third and fourth position in the operator $\mathcal{O}^{\chi \chi} \chi \chi \chi$. For example, for ${ }^{1} S_{0} \chi^{0} \chi^{+} \rightarrow \chi^{0} \chi^{+}$ operators one of the three classes of field-interchanged operators is given by the ${ }^{1} S_{0}$ $\chi^{+} \chi^{0} \rightarrow \chi^{+} \chi^{0}$ operators $\zeta_{e_{4}}^{\dagger} \xi_{e_{3}}^{c} \xi_{e_{2}}^{c \dagger} \zeta_{e_{1}}$.

and take the values 1 to $n_{0}$ for neutralino species and 1 to $n_{+}$for chargino species. Note that the order of the labels $e_{i}$ on the Wilson coefficients and the operators is not accidental in (6). The labels on the operators are given in the order, in which the field operators with label $e_{i}$ occur in the operator. In case of the corresponding Wilson coefficients, the indices refer to the actual scattering reaction $\chi_{e_{1}} \chi_{e_{2}} \rightarrow \chi_{e_{4}} \chi_{e_{3}}$, that is described by the operators. For the basis of the operators see Tab. 2. The $\chi$ in the labels $\chi \chi \rightarrow \chi \chi$ of the operators and Wilson coefficients in (6) should indicate the particular particle species $\chi^{0}$ and $\chi^{ \pm}$, whose $\chi_{e_{1}} \chi_{e_{2}} \rightarrow \chi_{e_{4}} \chi_{e_{3}}$ scattering reaction is described, see Tab. 1. A summation over the indices $e_{i}$ is implicit in (6). The normalization factor $1 / 4$ in (6) ensures that the tree-level transition matrix element for ${ }^{1} S_{0}$-wave scattering is given by

$$
\begin{aligned}
& \left.\left\langle\chi_{l} \chi_{k}\left|\int d^{4} x \sum_{\chi \chi \rightarrow \chi \chi} \frac{1}{4} f_{\left\{e_{1} e_{2}\right\}\left\{e_{4} e_{3}\right\}}^{\chi \chi \rightarrow \chi \chi}\left({ }^{1} S_{0}\right) \mathcal{O}_{\left\{e_{4} e_{3}\right\}\left\{e_{2} e_{1}\right\}}^{\chi \chi \rightarrow \chi \chi}\left({ }^{1} S_{0}\right)(x)\right| \chi_{i} \chi_{j}\right\rangle\right|_{\text {tree }} \\
& =(2 \pi)^{4} \delta^{(4)}\left(p_{\text {in }}-p_{\text {out }}\right) 2 f_{\{i j\}\{l k\}}^{\chi \chi \rightarrow \chi \chi}\left({ }^{1} S_{0}\right)
\end{aligned}
$$

for all $\chi_{i} \chi_{j} \rightarrow \chi_{l} \chi_{k}$ reactions at leading order in the non-relativistic effective theory. In (7) we have assumed that the incoming and outgoing two-particle states $\chi_{i} \chi_{j}$ and $\chi_{l} \chi_{k}$ both reside in a ${ }^{1} S_{0}$-wave configuration with normalised spin state $\frac{1}{\sqrt{2}}(|\uparrow \downarrow\rangle-|\downarrow \uparrow\rangle)$. A 
similar relation for the tree-level transition matrix element of ${ }^{3} S_{1}$-wave scattering in the effective theory holds for all $\chi_{i} \chi_{j} \rightarrow \chi_{l} \chi_{k}$ reactions. Note that in order to derive (77) one has to take into account relations among Wilson coefficients of different operators, which will be deduced in the next paragraph.

There are redundancies in $\delta \mathcal{L}_{\text {ann }}^{d=6}$, (6) , as several operators can describe one specific scattering reaction with a $\chi_{e_{1}}$ and a $\chi_{e_{2}}\left(\chi_{e_{4}}\right.$ and $\left.\chi_{e_{3}}\right)$ particle in the initial (final) state. This redundancy is associated with operators that arise from interchanging the singleparticle field operators at the first and second and/or third and fourth position in a given $\mathcal{O}^{\chi \chi \rightarrow \chi \chi}$. The corresponding Wilson coefficients are related to each other, as they encode the same information on a given specific scattering reaction. Consequently, the redundancy manifests itself in symmetry relations among the Wilson coefficients under exchange of the labels $e_{1} \leftrightarrow e_{2}$ and/or $e_{4} \leftrightarrow e_{3}$. These relations read

$$
\begin{aligned}
& f_{\left\{e_{2} e_{1}\right\}\left\{e_{4} e_{3}\right\}}^{\chi_{e_{2}} \chi_{e_{1}} \rightarrow \chi_{e_{1}} \chi_{e_{3}}}\left({ }^{2 s+1} S_{J}\right)=\eta_{s} f_{\left\{e_{1} e_{2}\right\}\left\{e_{4} e_{3}\right\}}^{\chi_{e_{1}} \chi_{e_{2}} \rightarrow \chi_{e_{4}} \chi_{e_{3}}}\left({ }^{2 s+1} S_{J}\right) \\
& f_{\left\{e_{1} e_{2}\right\}\left\{e_{3} e_{4}\right\}}^{\chi_{e_{1}} \chi_{e_{2}} \rightarrow \chi_{e_{3}} \chi_{e_{4}}}\left({ }^{2 s+1} S_{J}\right)=\eta_{s} f_{\left\{e_{1} e_{2}\right\}\left\{e_{4} e_{3}\right\}}^{\chi_{e_{1}} \chi_{e_{2}} \rightarrow \chi_{e_{4}} \chi_{e_{3}}}\left({ }^{2 s+1} S_{J}\right)
\end{aligned}
$$

with

$$
\eta_{s}=\left\{\begin{array}{rl}
1 & \text { for } s=0 \\
-1 & \text { for } s=1
\end{array} .\right.
$$

To exemplify the origin of the first relation in (8), let us consider the terms in $\delta \mathcal{L}_{\text {ann }}^{\mathrm{d}=6}$ that account for $S$-wave $\chi^{0} \chi^{0} \rightarrow \chi \chi$ reactions at leading order in the non-relativistic velocity expansion:

$$
\sum_{e_{1}, \ldots, e_{4}} \frac{1}{4} f_{\left\{e_{1} e_{2}\right\}\left\{e_{4} e_{3}\right\}}^{\chi^{0} \chi^{0} \rightarrow \chi \chi}\left({ }^{2 s+1} S_{J}\right) \mathcal{O}_{\left\{e_{4} e_{3}\right\}}^{(s) \chi \chi} \xi_{e_{2}}^{c \dagger} \Gamma^{(s)} \xi_{e_{1}}
$$

where the operator $\mathcal{O}_{\left\{e_{4} e_{3}\right\}}^{(s) \chi \chi}$ stands for the two-field operator that creates the outgoing state, and $\Gamma^{(s)}$ is given by $\Gamma^{(s=0)}=1_{2 \times 2}$ and $\Gamma^{(s=1)}=\vec{\sigma}$ in case of ${ }^{1} S_{0}$ and ${ }^{3} S_{1}$ operators, respectively. Using the definition of the spinor $\xi^{c}$ we can write

$$
\xi_{e_{2}}^{c \dagger} \Gamma^{(s)} \xi_{e_{1}}=\xi_{e_{1}}^{c \dagger} \sigma^{2} \Gamma^{(s) \top} \sigma^{2} \xi_{e_{2}}=\eta_{s} \xi_{e_{1}}^{c \dagger} \Gamma^{(s)} \xi_{e_{2}}
$$

with $\eta_{s}$ defined as in (9). After renaming the labels $e_{1}$ and $e_{2}$, the terms in (10) can be written as

$$
\sum_{e_{1}, \ldots, e_{4}} \frac{1}{4} \eta_{s} f_{\left\{e_{2} e_{1}\right\}\left\{e_{4} e_{3}\right\}}^{\chi^{0} e^{0} \rightarrow \chi \chi}\left({ }^{2 s+1} S_{J}\right) \quad \mathcal{O}_{\left\{e_{4} e_{3}\right\}}^{(s) \chi \chi} \xi_{e_{2}}^{c \dagger} \Gamma^{(s)} \xi_{e_{1}}
$$

Comparing to the original expression in (10), we arrive at the relation

$$
f_{\left\{e_{2} e_{1}\right\}\left\{e_{4} e_{3}\right\}}^{\chi^{0} \chi^{0} \rightarrow \chi \chi}\left({ }^{2 s+1} S_{J}\right)=\eta_{s} f_{\left\{e_{1} e_{2}\right\}\left\{e_{4} e_{3}\right\}}^{\chi^{0}{ }^{0} \rightarrow \chi \chi}\left({ }^{2 s+1} S_{J}\right) .
$$

This equation as well as the more comprehensive relations in (8) imply that the Wilson coefficients of ${ }^{1} S_{0}$ operators have to be symmetric under the exchange $e_{1} \leftrightarrow e_{2}$, whereas 
Wilson coefficients of ${ }^{3} S_{1}$ operators are antisymmetric under $e_{1} \leftrightarrow e_{2}$. The same statement applies to the exchange $e_{3} \leftrightarrow e_{4}$ in case of outgoing states. In processes with identical incoming or outgoing particles, the above relations in (8) imply the vanishing of the ${ }^{3} S_{1}$ Wilson coefficients. This rephrases the well-known fact that a pair of identical spin-1/2 particles cannot build a ${ }^{3} S_{1}$ state.

Finally, a further property of the Wilson coefficients under the exchange of the particle labels is directly inherited from the hermiticity of the non-relativistic Lagrangian:

$$
f_{\left\{e_{1} e_{2}\right\}\left\{e_{4} e_{3}\right\}}^{\chi \chi \rightarrow \chi \chi}\left({ }^{2 s+1} S_{J}\right)=\left[f_{\left\{e_{4} e_{3}\right\}\left\{e_{1} e_{2}\right\}}^{\chi \chi \rightarrow \chi \chi}\left({ }^{s+1} S_{J}\right)\right]^{*} .
$$

\subsection{Matching condition}

The Wilson coefficients of the four-fermion operators in $\delta \mathcal{L}_{\text {ann }}$ are determined by the matching condition

$$
\begin{aligned}
\mathcal{A}\left(\chi_{i} \chi_{j} \rightarrow \chi_{l} \chi_{k}\right) & \mid \text { MSSM, perturbative }=\sum \frac{1}{4} f_{\left\{e_{1} e_{2}\right\}\left\{e_{4} e_{3}\right\}}^{\chi \chi \rightarrow \chi \chi}\left({ }^{2 s+1} L_{J}\right) \\
& \times\left\langle\chi_{l} \chi_{k}\left|\mathcal{O}_{\left\{e_{4} e_{3}\right\}\left\{e_{2} e_{1}\right\}}^{\chi \chi \rightarrow \chi \chi}\left({ }^{2 s+1} L_{J}\right)\right| \chi_{i} \chi_{j}\right\rangle \mid \text { NRMSSM, perturbative }
\end{aligned}
$$

For this equation to hold, we have to use the same (non-relativistic) normalization of the incoming and outgoing states in both the full theory and the NRMSSM. Here we will determine the contributions to the Wilson coefficients that describe the tree-level annihilation reactions into exclusive SM and light Higgs two-body final states $X_{A} X_{B}$, which

we shall denote as $\hat{f} \chi \chi \rightarrow X_{A} X_{B} \rightarrow \chi \chi\left({ }^{2 s+1} L_{J}\right)$. The unitarity of the S-matrix at the diagrammatic level establishes a relation among the tree-level annihilation rate for $\chi_{i} \chi_{j} \rightarrow X_{A} X_{B}$ and the imaginary part of the 1-loop forward-scattering reaction $\chi_{i} \chi_{j} \rightarrow X_{A} X_{B} \rightarrow \chi_{i} \chi_{j}$ :

$$
\begin{aligned}
& \int\left[\mathrm{dPS}_{A B}\right]\left|\mathcal{A}\left(\chi_{i} \chi_{j} \rightarrow X_{A} X_{B}\right)\right|^{2}=2 \Im\left[\mathcal{A}\left(\chi_{i} \chi_{j} \rightarrow X_{A} X_{B} \rightarrow \chi_{i} \chi_{j}\right)\right] \\
& \left.=2 \sum \frac{1}{4} \Im\left[f_{\left\{e_{1} e_{2}\right\}\left\{e_{4} e_{3}\right\}}^{\chi \chi \rightarrow X_{A} X_{B} \rightarrow \chi \chi}\left({ }^{2 s+1} L_{J}\right)\right]\left\langle\chi_{i} \chi_{j}\right| \mathcal{O}_{\left\{e_{4} e_{3}\right\}\left\{e_{2} e_{1}\right\}}^{\chi \chi \rightarrow \chi \chi}{ }^{2 s+1} L_{J}\right)\left|\chi_{i} \chi_{j}\right\rangle .
\end{aligned}
$$

We generalize this and define the absorptive part of amplitude $\mathcal{A}\left(\chi_{i} \chi_{j} \rightarrow X_{A} X_{B} \rightarrow \chi_{l} \chi_{k}\right)$ as well as the absorptive part of the Wilson coefficients in the following way:

$$
\begin{aligned}
& \int\left[\mathrm{dPS}_{A B}\right] \mathcal{A}\left(\chi_{i} \chi_{j} \rightarrow X_{A} X_{B}\right) \times \mathcal{A}\left(\chi_{l} \chi_{k} \rightarrow X_{A} X_{B}\right)^{*} \\
& \quad=\left.2\left[\mathcal{A}\left(\chi_{i} \chi_{j} \rightarrow X_{A} X_{B} \rightarrow \chi_{l} \chi_{k}\right)\right]\right|_{\text {absorptive }} \\
& \quad=2 \sum \frac{1}{4} \hat{f} \hat{f}_{\left\{e_{1} e_{2}\right\}\left\{e_{4} e_{3}\right\}}^{\chi \chi} \rightarrow X_{e} \rightarrow \chi\left({ }^{2 s+1} L_{J}\right)\left\langle\chi_{l} \chi_{k}\left|\mathcal{O}_{\left\{e_{4} e_{3}\right\}\left\{e_{2} e_{1}\right\}}^{\chi \chi}\left({ }^{2 s+1} L_{J}\right)\right| \chi_{i} \chi_{j}\right\rangle
\end{aligned}
$$

where we have introduced the notation

$$
\hat{f}_{\{i j\}\{l k\}}^{\chi \chi \rightarrow X_{A} X_{B} \rightarrow \chi \chi}\left({ }^{2 s+1} L_{J}\right)=\left.f_{\{i j\}\{l k\}}^{\chi \chi \rightarrow X_{A} X_{B} \rightarrow \chi \chi}\left({ }^{2 s+1} L_{J}\right)\right|_{\text {absorptive }} .
$$


With this definition, the absorptive part of a Wilson coefficient that encodes a $\chi_{i} \chi_{j} \rightarrow$ $\chi_{i} \chi_{j}$ forward-scattering reaction coincides with its imaginary part.

We make use of the defining relations to determine the absorptive part of the Wil-

son coefficients $\hat{f} \chi \chi \rightarrow X_{A} X_{B} \rightarrow \chi \chi$ from the product of the full-theory tree-level annihilation amplitudes integrated over the final state particles' phase-space, as given in the first line of (16). Technically this is achieved by considering all 1-loop scattering amplitudes $\chi_{i} \chi_{j} \rightarrow X_{A} X_{B} \rightarrow \chi_{l} \chi_{k}$ with a specific SM or Higgs particle-pair $X_{A} X_{B}$ in the intermediate state and by applying the Cutkosky rules to the $X_{A}$ and $X_{B}$ propagators. The resulting expression coincides with the first line of (16). To determine the absorptive part of the Wilson coefficients, the expression has to be expanded in the non-relativistic momenta of the external particles as well as in their mass differences and an appropriate spin-projection has to be performed.

\subsection{Expansion in mass differences in $\delta \mathcal{L}_{\text {ann }}$}

To simplify the notation, we shall replace the indices $\left(e_{1}, e_{2}, e_{3}, e_{4}\right)$ by $(1,2,3,4)$ throughout this section. Further, we shall adopt the convention that particles 1 and 4 in the reaction $\chi_{1} \chi_{2} \rightarrow \chi_{4} \chi_{3}$ share the same reference mass scale $m$, while particles 2 and 3 have masses closer to the reference scale $\bar{m}$. Introducing two distinct mass scales for the particle species allows us to consider pair annihilations of two particles with similar mass $(m \sim \bar{m})$, but also pair annihilation of a hydrogen-like two-particle system where one of the particles is much lighter (though still heavy enough to be considered as non-relativistic). According to these assignments, we define

$$
\begin{array}{ll}
m_{1}=m-\delta m, & m_{2}=\bar{m}-\delta \bar{m}, \\
m_{4}=m+\delta m, & m_{3}=\bar{m}+\delta \bar{m},
\end{array}
$$

with

$$
m=\frac{m_{1}+m_{4}}{2}, \quad \bar{m}=\frac{m_{2}+m_{3}}{2},
$$

such that the mass differences read

$$
\delta m=\frac{m_{4}-m_{1}}{2}, \quad \delta \bar{m}=\frac{m_{3}-m_{2}}{2} .
$$

The results for the Wilson coefficients presented in the appendix adopt the definitions (18 20). If for a given process $\chi_{i} \chi_{j} \rightarrow \chi_{l} \chi_{k}$ it turns out that the reverse condition, $m_{i} \sim m_{k} \sim m$ and $m_{j} \sim m_{l} \sim \bar{m}$, is more meaningful given the actual values of the masses, one can make use of the symmetry properties (8) to relate the Wilson coefficients for $\chi_{i} \chi_{j} \rightarrow \chi_{l} \chi_{k}$ to those of $\chi_{i} \chi_{j} \rightarrow \chi_{k} \chi_{l}$, which would then conform to the prescription above, i.e. $m$ would be equal to the average of the mass of the particle associated with field 1 and the mass of the particle associated with field $4, m=\left(m_{i}+m_{k}\right) / 2$. Note that the mass differences $\delta m$ and $\delta \bar{m}$ in (20) obviously vanish in case of diagonal scattering reactions $\chi_{1} \chi_{2} \rightarrow \chi_{1} \chi_{2}$, such that $m=m_{1}$ and $\bar{m}=m_{2}$ in that case. 
The absorptive parts of the Wilson coefficients are obtained by matching amplitudes for the process $\chi_{1} \chi_{2} \rightarrow \chi_{4} \chi_{3}$ with on-shell external states. This implies that the energyconservation relation in the center-of-mass system,

$$
\sqrt{s}=E_{1}\left(\vec{p}^{2}\right)+E_{2}\left(\vec{p}^{2}\right)=E_{4}\left(\vec{p}^{\prime 2}\right)+E_{3}\left(\vec{p}^{\prime 2}\right),
$$

with $E_{i}\left(\vec{p}^{2}\right)=\sqrt{m_{i}^{2}+\vec{p}^{2}}$ and $\vec{p}\left(\vec{p}^{\prime}\right)$ the incoming (outgoing) particles' momentum in the center-of-mass system, is fulfilled. Using (18) and $M \equiv m+\bar{m}$, the expansion of the energy-conservation relation (21) for non-relativistic momenta $\vec{p}^{2}$ and $\vec{p}^{2}$ reads

$$
\sqrt{s}=M-\delta m-\delta \bar{m}+\frac{\vec{p}^{2}}{2 \mu}+\ldots=M+\delta m+\delta \bar{m}+\frac{\vec{p}^{\prime 2}}{2 \mu}+\ldots,
$$

where $\mu=m \bar{m} / M$ and terms of order $\vec{p}^{4} / \mu^{3}$ and $\left(\delta m / M \times \vec{p}^{2} / \mu\right)$ have been dropped. This can be rewritten as

$$
\frac{\vec{p}^{\prime 2}}{2 \mu}=\frac{\vec{p}^{2}}{2 \mu}-2 \delta m-2 \delta \bar{m}+\ldots .
$$

From (23) we see that a consistent expansion which treats both $\vec{p}^{2}$ and $\vec{p}^{\prime 2}$ as small quantities of the same order requires that the mass differences $\delta m, \delta \bar{m}$ are also formally considered of order $\vec{p}^{2} / \mu$ in the expansion of the amplitudes. Note that an expansion in mass differences is only required for the off-diagonal scattering reactions where the incoming and outgoing $\chi \chi$ states are different, as $\delta m=\delta \bar{m}=0$ for $\chi_{1} \chi_{2} \rightarrow \chi_{1} \chi_{2}$ reactions.

The amplitude for a generic process $\chi_{1} \chi_{2} \rightarrow X_{A} X_{B} \rightarrow \chi_{4} \chi_{3}$ then depends on the hard scales $(m, \bar{m})$ and on the small scales $\left(\vec{p}^{2} / \mu, \vec{p}^{\prime 2} / \mu, \vec{p} \cdot \vec{p}^{\prime} / \mu, \delta m, \delta \bar{m}\right) \sim \mathcal{O}\left(\mu v^{2}\right)$, where $v$ stands for the relative velocity in the two-particle system. In the following we enumerate the steps to obtain the absorptive part of the Wilson coefficients from the process $\chi_{1} \chi_{2} \rightarrow X_{A} X_{B} \rightarrow \chi_{4} \chi_{3}$, including the subleading $\mathcal{O}\left(v^{2}\right)$ terms, which will be presented in [23].

1. The absorptive part of the 1-loop scattering amplitude $\chi_{1} \chi_{2} \rightarrow X_{A} X_{B} \rightarrow \chi_{4} \chi_{3}$ with a SM or Higgs final state $X_{A} X_{B}$ is computed by applying the Cutkosky rules to the $X_{A}$ and $X_{B}$ propagators. The result is written in terms of the mass scales introduced above, and expanded in the small scales retaining terms up to $\mathcal{O}\left(v^{2}\right)$.

2. To $\mathcal{O}\left(v^{2}\right)$ the result contains scalar products with at most two powers of $\vec{p}$ and $\vec{p}^{\prime}$. For the spin-1 configuration, the scalar products also involve the spin-polarization vectors $\vec{n}$ and $\vec{n}^{\prime}$ of the incoming $\left(\chi_{1} \chi_{2}\right)$ and outgoing $\left(\chi_{4} \chi_{3}\right)$ states, respectively. The generic form of the result for spin-1 incoming and outgoing states reads

$$
\begin{aligned}
& \left\{c_{0}\left({ }^{3} S_{1}\right)+c_{1}\left({ }^{3} S_{1}\right) \delta m+c_{2}\left({ }^{3} S_{1}\right) \delta \bar{m}+c_{3}\left({ }^{3} S_{1}\right) \vec{p}^{2}+c_{4}\left({ }^{3} S_{1}\right) \vec{p}^{\prime 2}\right\} \vec{n} \cdot \vec{n}^{\prime} \\
& +c_{5}\left({ }^{3} P_{0}\right)(\vec{p} \cdot \vec{n})\left(\vec{p}^{\prime} \cdot \vec{n}^{\prime}\right)+c_{6}\left({ }^{3} P_{1}\right)[p, n]^{k}\left[p^{\prime}, n^{\prime}\right]^{k}+c_{7}\left({ }^{3} P_{2}\right) p^{\{i} n^{j\}} p^{\prime\{i} n^{\prime j\}} \\
& +c_{8}\left({ }^{3} S_{1},{ }^{3} P_{1}\right) n^{k}\left[p^{\prime}, n^{\prime}\right]^{k}+c_{9}\left({ }^{3} P_{1},{ }^{3} S_{1}\right)[p, n]^{k} n^{\prime k}
\end{aligned}
$$




$$
+c_{10}\left({ }^{3} S_{1},{ }^{3} D_{1}\right) p^{\prime\{i} p^{\prime j\}} n^{i} n^{\prime j}+c_{11}\left({ }^{3} D_{1},{ }^{3} S_{1}\right) p^{\{i} p^{j\}} n^{i} n^{\prime j},
$$

where we have introduced the notation $[a, b]^{k} \equiv \varepsilon^{i j k} a^{i} b^{j}$ and $a^{\{i} b^{j\}} \equiv a^{i} b^{j}+a^{j} b^{i}-$ $2 \vec{a} \cdot \vec{b} \delta^{i j} / 3$, corresponding to $J=1$ and $J=2$ Cartesian tensors, respectively. The spin-polarization vector $\vec{n}$ is introduced by replacing the spinor matrix $\left[\xi \xi^{c \dagger}\right]_{i j}$ of an incoming two-neutralino state by $\frac{1}{\sqrt{2}} \vec{n} \cdot \vec{\sigma}_{i j}$. Similar replacements apply to outgoing two-particle states and states involving charginos. The coefficients $c_{i}$ are functions of $m$ and $\bar{m}$. The first term, $c_{0}$, gives the leading-order contribution, where all the others count as $\mathcal{O}\left(v^{2}\right)$. We have further specified the quantum numbers ${ }^{3} L_{J}$ of each term, which matches the angular-momentum configuration of the incoming state, equal to that of the outgoing state except for the $c_{8-11}$ terms (the first quantum number between parentheses refers then to the incoming state, the second to the outgoing one). For spin-0 incoming and outgoing states, the result simplifies to

$$
c_{0}\left({ }^{1} S_{0}\right)+c_{1}\left({ }^{1} S_{0}\right) \delta m+c_{2}\left({ }^{1} S_{0}\right) \delta \bar{m}+c_{3}\left({ }^{1} S_{0}\right) \vec{p}^{2}+c_{4}\left({ }^{1} S_{0}\right) \vec{p}^{\prime 2}+c_{5}\left({ }^{1} P_{1}\right) \vec{p} \cdot \vec{p}^{\prime} .
$$

We have not considered the possibility of spin- 0 to spin- 1 transitions between incoming and outgoing states in the hard annihilation process, though the transitions ${ }^{3} S_{1} \rightarrow{ }^{1} P_{1}$ and ${ }^{3} P_{0,1} \rightarrow{ }^{1} S_{0},{ }^{1} P_{1}$ are also allowed at $\mathcal{O}(v)$ by angular-momentum conservation. Such spin-changing transitions in the hard annihilation part of the full forward scattering amplitude (see Fig. (1) will also require spin-changing potential interactions in the long-range part, in order to bring the spin of the two-particle state after annihilation back to the spin of the (left-most) incoming state. Since the non-relativistic spin-changing potentials carry an additional $v$-suppression, such transitions are only relevant for the calculation of the annihilation rates at $\mathcal{O}\left(g^{2} v^{2}\right)$. At present we ignore $\mathcal{O}\left(v^{2}\right)$ effects that arise from subleading non-Coulomb (nonYukawa) potentials and consider only those from the short-distance annihilation. Likewise, the terms $c_{8-11}$ included in (24) imply a change of the orbital angular momentum which must be compensated by a potential interaction which is also $v$-suppressed in the non-relativistic limit, and can be ignored for our purposes.

3. By virtue of the energy-conservation relation (22), we rewrite powers of $\vec{p}^{2}$ and $\vec{p}^{\prime 2}$ as

$$
\begin{aligned}
\vec{p}^{2} & =\frac{1}{2}\left(\vec{p}^{2}+\vec{p}^{\prime 2}\right)+\frac{2 m \bar{m}}{M}(\delta m+\delta \bar{m})+\ldots, \\
\vec{p}^{\prime 2} & =\frac{1}{2}\left(\vec{p}^{2}+\vec{p}^{\prime 2}\right)-\frac{2 m \bar{m}}{M}(\delta m+\delta \bar{m})+\ldots,
\end{aligned}
$$

such that the coefficients multiplying $\vec{p}^{2}$ and $\vec{p}^{\prime 2}$ become equal. This convention is adopted in order that the Wilson coefficients of the dimension- 8 operators with derivatives also have the symmetry property (13) under the exchange of the incoming and outgoing states. 
4. Finally, the Wilson coefficients are identified by comparing the expanded expression for the absorptive part of the amplitude $\mathcal{A}\left(\chi_{1} \chi_{2} \rightarrow X_{A} X_{B} \rightarrow \chi_{4} \chi_{3}\right)$ with the amplitude for the same process computed with the dimension 6 and dimension 8 EFT operators in $\delta \mathcal{L}_{\text {ann }}$.

The explicit expressions for the leading-order $S$-wave coefficients are provided in the appendix. While the Wilson coefficients refer to the inclusive annihilation rates, summed over all accessible final states, the calculation is performed for individual final states, which are therefore also given separately. Such final-state separated results should be of interest to the calculation of primary decay spectra of dark matter annihilation in the present Universe.

\section{Results}

We have performed a number of dedicated numeric and analytic checks of our results for the absorptive parts of the Wilson coefficients. As these expressions also encode the absorptive part of $\chi_{e_{1}} \chi_{e_{2}} \rightarrow X_{A} X_{B} \rightarrow \chi_{e_{1}} \chi_{e_{2}}$ forward scattering reactions, which are related to the tree-level annihilation cross section for $\chi_{e_{1}} \chi_{e_{2}} \rightarrow X_{A} X_{B}$ processes (see (15)), a comparison of the analytic non-relativistic approximation to the tree-level annihilation cross section with results from a numeric code can be carried out for the diagonal entries of the annihilation coefficients. We discuss this in Sec. 3.1.

In addition, we can relate our analytic expressions for partial-wave separated neutralino LSP pair-annihilation cross sections to existing analytic results available in the literature [13, 14, 22. This will be briefly discussed in Sec. 3.2. No checks are available in the general case for the off-diagonal entries of the annihilation coefficient matrix.

\subsection{Numerical comparison with MADGRAPH}

The expansion of the exclusive, spin-averaged center-of-mass frame $\chi_{e_{1}} \chi_{e_{2}} \rightarrow X_{A} X_{B}$ tree-level pair-annihilation cross section in the non-relativistic momentum $\vec{p}$ of the $\chi_{e_{i}}$ particles is given by

$$
\begin{aligned}
& \sigma^{\chi_{e_{1}} \chi_{e_{2}} \rightarrow X_{A} X_{B}} v_{\text {rel }}=\hat{f}\left({ }^{1} S_{0}\right)+3 \hat{f}\left({ }^{3} S_{1}\right) \\
& +\frac{\vec{p}^{2}}{M^{2}}\left(\hat{f}\left({ }^{1} P_{1}\right)+\frac{1}{3} \hat{f}\left({ }^{3} P_{0}\right)+\hat{f}\left({ }^{3} P_{1}\right)+\frac{5}{3} \hat{f}\left({ }^{3} P_{2}\right)+\hat{g}\left({ }^{1} S_{0}\right)+3 \hat{g}\left({ }^{3} S_{1}\right)\right)+\mathcal{O}\left(\vec{p}^{4}\right) .
\end{aligned}
$$

Here $v_{\text {rel }}=\left|\vec{v}_{e_{1}}-\vec{v}_{e_{2}}\right|$ is the relative velocity of the $\chi_{e_{1}} \chi_{e_{2}}$ pair and $\vec{v}_{e_{i}}$ denotes the velocity of particle $\chi_{e_{i}}$ in the center-of-mass frame of the annihilation reaction. We have suppressed the superscripts $\chi_{e_{1}} \chi_{e_{2}} \rightarrow X_{A} X_{B} \rightarrow \chi_{e_{1}} \chi_{e_{2}}$ on the Wilson coefficients $\hat{f}$ in (27), where these expressions explicitly refer to the exclusive (tree-level) annihilation rates. Further note that (27) contains not only the leading-order $S$-wave Wilson coefficients $\hat{f}\left({ }^{2 s+1} S_{J}\right)$ with spin configuration $s=0,1$ of the incoming two-body system, but also 
includes $P$-wave and next-to-next-to-leading order $S$-wave coefficients (denoted with $\hat{g}$ ). For analytic results on those coefficients we refer the reader to [23].

In the non-relativistic limit the relation between the relative velocity $v_{\text {rel }}$ and the particle momentum $\vec{p}$ in the center-of-mass frame of the $\chi_{e_{1}} \chi_{e_{2}}$ annihilation reaction is approximated by

$$
v_{\text {rel }}=\left|\vec{v}_{e_{1}}-\vec{v}_{e_{2}}\right|=|\vec{p}|\left(\frac{m_{e_{1}}+m_{e_{2}}}{m_{e_{1}} m_{e_{2}}}+\mathcal{O}\left(\vec{p}^{2}\right)\right)
$$

Together with (27), this relation allows us to express the first two coefficients, $a$ and $b$, in the Taylor expansion of the $\chi_{e_{1}} \chi_{e_{2}} \rightarrow X_{A} X_{B}$ center-of-mass frame annihilation cross section with respect to the relative velocity,

$$
\sigma^{\chi_{e_{1}} \chi_{e_{2}} \rightarrow X_{A} X_{B}} v_{\mathrm{rel}}=a+b v_{\mathrm{rel}}^{2}+\mathcal{O}\left(v_{\mathrm{rel}}^{4}\right)
$$

in terms of the partial-wave separated Wilson coefficients $\hat{f} \chi_{e_{1}} \chi_{e_{2}} \rightarrow X_{A} X_{B} \rightarrow \chi_{e_{1}} \chi_{e_{2}}\left({ }^{2 s+1} L_{J}\right)$

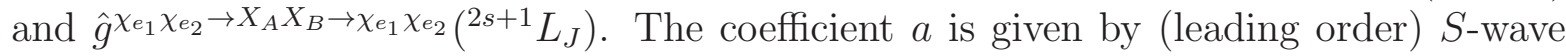
Wilson coefficients only,

$$
a=\hat{f}\left({ }^{1} S_{0}\right)+3 \hat{f}\left({ }^{3} S_{1}\right) .
$$

The coefficient $b$ receives both $P$-wave and next-to-next-to-leading order $S$-wave Wilson coefficient contributions,

$$
\begin{gathered}
b=\frac{m_{e_{1}}^{2} m_{e_{2}}^{2}}{M^{2}\left(m_{e_{1}}+m_{e_{2}}\right)^{2}}\left(\hat{f}\left({ }^{1} P_{1}\right)+\frac{1}{3} \hat{f}\left({ }^{3} P_{0}\right)+\hat{f}\left({ }^{3} P_{1}\right)+\frac{5}{3} \hat{f}\left({ }^{3} P_{2}\right)\right. \\
\left.+\hat{g}\left({ }^{1} S_{0}\right)+3 \hat{g}\left({ }^{3} S_{1}\right)\right) .
\end{gathered}
$$

The parameters $a$ and $b$ in (29) can also be extracted numerically from computer codes that determine the center-of-mass frame annihilation cross sections. This is done by considering the cross section's behaviour for small relative velocities of the annihilating particle pair and performing a parabola fit to $\sigma^{\chi_{e_{1}} \chi_{e_{2}} \rightarrow X_{A} X_{B}} v_{\text {rel }}$, which provides the corresponding coefficients $a$ and $b$. Note, however, that a separation of the coefficient $b$ into its constituent $P$-wave and next-to-next-to-leading order $S$-wave contributions, as given in (31), cannot be achieved with the sole knowledge of the cross section. Likewise, the separation of the $S$-wave contributions for the spin singlet and triplet configurations, as performed in (30) and (31), requires intervention at the amplitude level, which is not straightforward for the publicly available computer codes.

In the absence of threshold effects, resonances or enhanced radiative corrections, the knowledge of the coefficients $a$ and $b$ in $\chi_{e_{1}} \chi_{e_{2}}$ annihilation processes allows for a rather accurate calculation of the present-day relic abundance. Yet the separation of $b$ into $P$ - and $S$-wave contributions is required for a consistent treatment of the Sommerfeld enhancement at $\mathcal{O}\left(v^{2}\right)$ because the long-range interactions responsible for 
the Sommerfeld effect depend on the quantum numbers of the incoming state. Our analytic approach allows us to perform this separation by construction.

We perform a numeric check of our results for the $\chi_{e_{1}} \chi_{e_{2}} \rightarrow X_{A} X_{B}$ tree-level annihilation cross sections as given in (27) for all initial state two-particle pairs in Tab. 1 into all accessible SM and Higgs two-particle final states. We consider several MSSM spectra, which we compute using the spectrum calculator SuSpect [25] and its implementation of the phenomenological MSSM, a model with 27 free parameters. For each spectrum, we obtain the coefficients $a$ and $b$ in (30) and (31) from our analytic calculation, and compare them with the corresponding coefficients extracted purely numerically using MADGRAPH [26] to calculate the cross sections. Our results for the coefficient $a$ agree with the corresponding numeric expression extracted from MADGRAPH data at permille level. Similarly, we find agreement of the coefficients $b$ derived with (31) and extracted from MADGRAPH data at 1\% up to permille level, where the level of agreement slightly varies depending on the initial- and final-state particles. In addition, the level of agreement on the parameter $b$ depends on the interval of the $v_{\text {rel }}$ variable used for the parabola fit to the MADGRAPH data, which for the numbers quoted above is taken as $v_{\text {rel }} / c=[0,0.4]$. We find that the non-relativistic approximation is reliable for single-particle velocities up to $v_{e_{i}} / c \sim 0.3$. For such velocities the absolute error of the non-relativistic approximation to $\sigma^{\chi_{e_{1}} \chi_{e_{2}} \rightarrow X_{A} X_{B}} v_{\text {rel }}$ with respect to the unexpanded $\sigma^{\chi_{e_{1}} \chi_{e_{2}} \rightarrow X_{A} X_{B}} v_{\text {rel }}$ expression lies within the level of a few percent. Therefore the non-relativistic approximation has an acceptable accuracy for calculations in the early Universe during the time of $\chi_{e_{i}}$-decoupling, as the mean velocity of the $\chi_{e_{i}}$ in that period was around $v_{e_{i}} / c \sim 0.2$.

Selected results of our numeric check with MADGRAPH are presented in Fig. 2 and Fig. 3, where the underlying SUSY spectrum contains a wino-like neutralino LSP with mass $m_{\chi_{1}^{0}}=2748.92 \mathrm{GeV}$ and an almost mass-degenerate wino-like chargino with $m_{\chi_{1}^{+}}=$ $2749.13 \mathrm{GeV}$. Fig. 2 shows tree-level annihilation cross sections that are relevant in the calculation of the neutralino LSP relic abundance including co-annihilations. The plot on the left-hand side displays the annihilation cross section times the relative velocity for the double-charged annihilation reaction $\chi_{1}^{+} \chi_{1}^{+} \rightarrow W^{+} W^{+}$. For $v_{\text {rel }} / c \lesssim 0.4$ our analytic, non-relativistic approximation nicely reproduces the numeric, unexpanded cross section $\sigma^{\chi_{1}^{+} \chi_{1}^{+} \rightarrow W^{+} W^{+}} v_{\text {rel }}$. Furthermore, as the absolute curvature in this $S$-wave dominated reaction is rather small compared to the coefficient $a$, even the absolute error that one would make in using the non-relativistic approximation instead of the full cross section is only of the order of $2 \%$ for $v_{\text {rel }} / c \sim 0.6$. The coefficient $b$ for this reaction, calculated using (31), is given by $b c^{2}=1.27 \cdot 10^{-27} \mathrm{~cm}^{3} \mathrm{~s}^{-1}$. Its $P$ - and $S$-wave contributions are of the same order and read $b_{P} c^{2}=2.95 \cdot 10^{-27} \mathrm{~cm}^{3} \mathrm{~s}^{-1}$ and $b_{S} c^{2}=-1.68 \cdot 10^{-27} \mathrm{~cm}^{3} \mathrm{~s}^{-1}$. The plot on the right-hand side in Fig. 2 depicts the single-charged annihilation reaction $\chi_{1}^{0} \chi_{1}^{+} \rightarrow t \bar{b}$ with (massive) fermionic final states. As it receives significant leading order $S$-wave contributions, this annihilation process is also relevant in the neutralino LSP relic abundance calculation including co-annihilation processes. Here it turns out that the $b$ coefficient is $S$-wave dominated, as the contributions from $P$-waves are suppressed by five orders of magnitude. Let us stress that our analytic results for the Wilson coefficients 

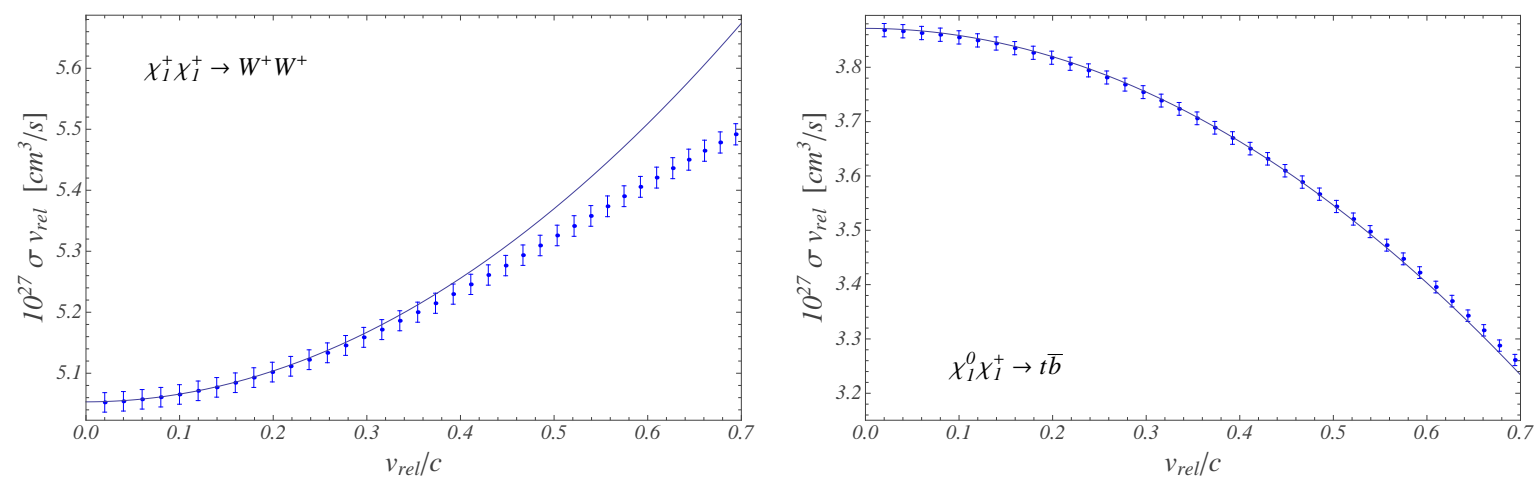

Figure 2: Numeric comparison of the non-relativistic approximation (solid lines) to the tree-level annihilation cross-section times relative velocity, $\sigma v_{\text {rel }}$, for $\chi_{1}^{+} \chi_{1}^{+} \rightarrow W^{+} W^{+}$ (left) and $\chi_{1}^{0} \chi_{1}^{+} \rightarrow t \bar{b}$ (right) reactions with the corresponding unexpanded annihilation cross section produced with MADGRAPH. The numeric errors on the latter are taken to be $\sigma v_{\text {rel }} / \sqrt{N}$, where $N=10^{5}$ gives the number of events used in the MADGRAPH calculation of each cross section value. $v_{\text {rel }}$ is given by $v_{\text {rel }}=\left|\vec{v}_{e_{1}}-\vec{v}_{e_{2}}\right|$ for the $\chi_{e_{1}} \chi_{e_{2}} \rightarrow$ $X_{A} X_{B}$ process. The underlying MSSM spectrum is a wino-like neutralino LSP scenario, generated with the spectrum calculator SuSpect. The masses of the $\chi_{1}^{0}$ and $\chi_{1}^{+}$are given by $m_{\chi_{1}^{0}}=2748.92 \mathrm{GeV}$ and $m_{\chi_{1}^{+}}=2749.13 \mathrm{GeV}$.

include the full mass-dependence of the final state particles and can be applied to MSSM scenarios with flavour off-diagonal sfermion generation mixing as well.

The plots in Fig. 3 show that our results can not only be used to describe pair annihilations of nearly mass-degenerate incoming particles $\chi_{e_{1}} \chi_{e_{2}} \rightarrow X_{A} X_{B}$, but also apply to annihilations of a non-relativistic "hydrogen-like" $\chi_{e_{1}} \chi_{e_{2}}$ two-particle system of non-degenerate-in-mass constituents. The plot on the left hand side in Fig. 3 corresponds to the pair annihilation of a hydrogen-like $\chi_{1}^{0} \chi_{3}^{0}$ state into a $W^{+} H^{-}$final state, with $m_{\chi_{3}^{0}}=3061.99 \mathrm{GeV}$, which is again dominated by leading-order $S$-wave contributions. The curvature is driven negative by the next-to-next-to-leading order $S$-wave contributions to the coefficient $b$, given by $b_{S} c^{2}=-5.29 \cdot 10^{-28} \mathrm{~cm}^{3} \mathrm{~s}^{-1}$. The $P$-wave contributions are, however, of the same order and read $b_{P} c^{2}=1.30 \cdot 10^{-28} \mathrm{~cm}^{3} \mathrm{~s}^{-1}$. The right plot in Fig. 3 again refers to a hydrogen-like incoming two-body system, $\chi_{1}^{+} \chi_{2}^{-}$, where in this case the annihilation $\chi_{1}^{+} \chi_{2}^{-} \rightarrow H^{+} H^{-}$is $P$-wave dominated: the $P$-wave contribution to the coefficient $b$ is given by $b_{P} c^{2}=2.48 \cdot 10^{-31} \mathrm{~cm}^{3} \mathrm{~s}^{-1}$. Both the leading and next-to-next-to-leading order $S$-wave contributions are strongly suppressed and of the order $\mathcal{O}\left(10^{-33} \mathrm{~cm}^{3} \mathrm{~s}^{-1}\right)$, respectively. The mass of the second chargino is given by $m_{\chi_{2}^{-}}=3073.31 \mathrm{GeV}$.

Generically, if the coefficient $a$ in the expansion (29) is suppressed with respect to the coefficient $b$, the curvature and hence the corresponding non-relativistic annihilation process is $P$-wave dominated. This property derives from the fact, that the leadingorder coefficient $a$ is related to the product of the leading order $S$-wave contributions to the tree-level annihilation amplitude with its complex conjugate. As the next-to- 

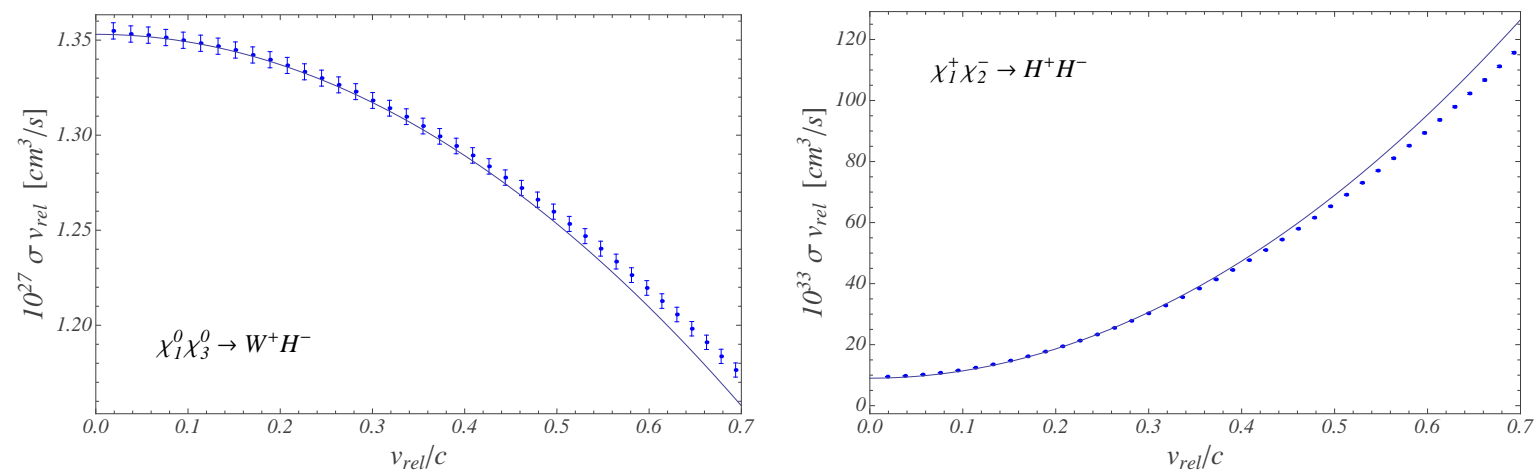

Figure 3: Numeric comparison of the non-relativistic approximation (solid line) to $\sigma v_{r e l}$ for the two neutral hydrogen-like two-body states $\chi_{1}^{0} \chi_{3}^{0} \rightarrow W^{+} H^{-}$(left) and $\chi_{1}^{+} \chi_{2}^{-} \rightarrow$ $H^{+} H^{-}$(right) to data produced with MAdGraph. Again, we take the errors on the MADGRAPH data to be $\sigma v_{\text {rel }} / \sqrt{N}$, where $N=10^{5}$ gives the number of events used in the MADGRAPH calculation of each cross section value. The process on the righthand side is dominated by $P$-wave annihilations. The underlying MSSM spectrum is the same as in the plots in Fig. 2, where the masses of the $\chi_{3}^{0}$ and $\chi_{2}^{-}$are given by $m_{\chi_{3}^{0}}=3061.99 \mathrm{GeV}$ and $m_{\chi_{2}^{-}}=3073.31 \mathrm{GeV}$. The mass of the Higgs particles $H^{ \pm}$takes the value $m_{H^{ \pm}}=167.29 \mathrm{GeV}$.

next-to-leading order $S$-wave contributions to the coefficient $b$ result from the product of leading order with next-to-next-to-leading order $S$-wave contributions in the annihilation amplitudes, a suppressed coefficient $a$ indicates a small next-to-next-to-leading order $S$ wave contribution to the coefficient $b$ as well.

\subsection{Analytic checks}

In [22], the authors performed a calculation of the neutralino relic abundance in minimal supergravity models. In the appendix, they give a complete summary of all partial-wave separated tree-level helicity amplitudes in $\chi_{1}^{0} \chi_{1}^{0} \rightarrow X_{A} X_{B}$ pair annihilations. These comprehensive results for tree-level neutralino LSP pair-annihilations are also referenced and (partly) quoted in the (SUSY) particle dark matter reviews [2] and [3], and easily extend to $\chi_{e_{1}}^{0} \chi_{e_{1}}^{0} \rightarrow X_{A} X_{B}$ annihilations. Hence, these results allow for an explicit analytic check of our expressions for the different partial-wave contributions to a neutralino $\chi_{e_{1}}^{0} \chi_{e_{1}}^{0} \rightarrow X_{A} X_{B}$ annihilation cross section. The partial-wave coefficients that can be cross-checked in that way correspond to ${ }^{1} S_{0^{-}},{ }^{3} P_{0^{-}},{ }^{3} P_{1^{-}}$and ${ }^{3} P_{2^{-}}$wave $\chi_{e_{1}}^{0} \chi_{e_{1}}^{0} \rightarrow X_{A} X_{B}$ annihilation reactions, and the leading order and next-to-next-to-leading order ${ }^{1} S_{0}$-wave contributions can be compared separately. As already inferred from (8) and noted at the end of Sec. 2.2, there are no ${ }^{3} S_{1}$ and ${ }^{1} P_{1}$ partial-wave contributions for annihilation reactions of identical incoming particles, which is the case covered by [22]. Our expressions for the partial-wave separated $\chi_{e_{1}}^{0} \chi_{e_{1}}^{0} \rightarrow X_{A} X_{B}$ annihilation cross sections into all possible SM and Higgs final states, obtained from (27), agree with the corre- 
sponding terms derived from the helicity amplitudes in $[22]^{1}$. We note that our results for annihilations into a pair of fermions include the case of flavour-off-diagonal sfermion mixing as well, which is covered in [2] and [3], but was not yet included in [22], wherein only flavour-diagonal right-left sfermion mixing was taken into account, although it is straightforward to extend these results to the general flavour-off-diagonal case.

The comparison with analytic results for inclusive leading-order ${ }^{1} S_{0^{-}}$and ${ }^{3} S_{1}$-wave pair-annihilation reactions of a pure wino-like neutralino $\chi_{1}^{0}$ and its mass-degenerate chargino partners $\chi_{1}^{ \pm}$into all possible SM and Higgs final states considered in Ref. [14] provides another useful check of our results for the absorptive part of the Wilson coefficients. The results in [14] comprise all possible neutral, as well as single and double charged inclusive pair-annihilation reactions. The masses of the SM and Higgs particle final states are set to zero, such that the corresponding results can be understood as the leading-order term in an expansion in $m_{\mathrm{SM}} / m_{\chi_{1}^{0}}$ and $m_{\mathrm{Higgs}} / m_{\chi_{1}^{0}}$. Furthermore, all supersymmetric particle states heavier than $\chi_{1}^{0}$ and $\chi_{1}^{ \pm}$are treated as completely decoupled. We agree with all results for the inclusive annihilation reactions given in [14]. In particular we agree with the results in [14] that refer to leading-order ${ }^{1} S_{0}$-wave $\chi_{1}^{0} \chi_{1}^{0} \rightarrow \chi_{1}^{-} \chi_{1}^{+}$ as well as $\chi_{1}^{-} \chi_{1}^{+} \rightarrow \chi_{1}^{0} \chi_{1}^{0}$ reactions, which can be related to the Wilson coefficients $\hat{f} \chi_{1}^{0} \chi_{1}^{0} \rightarrow \chi_{1}^{-} \chi_{1}^{+}\left({ }^{1} S_{0}\right)$ and $\hat{f} \chi_{1}^{-} \chi_{1}^{+} \rightarrow \chi_{1}^{0} \chi_{1}^{0}\left({ }^{1} S_{0}\right)$, therewith permitting an explicit check of some of our Wilson coefficients encoding off-diagonal scattering reactions.2

\section{Discussion}

\subsection{Unitary vs Feynman gauge}

The computation of the absorptive parts of the Wilson coefficients for forward-scattering reactions, $\chi_{e_{1}} \chi_{e_{2}} \rightarrow X_{A} X_{B} \rightarrow \chi_{e_{1}} \chi_{e_{2}}$, has been performed using both the unitary and Feynman gauge. The results agree numerically, which provides a further check of our calculation. For the off-diagonal reactions, where the incoming and outgoing states are different, the use of unitary gauge for final states with two massive vector bosons in the final state introduces enhanced $1 / M_{V}^{4}$ and $1 / M_{V}^{2}$ terms proportional to the mass differences between the incoming and outgoing particle species, which must cancel in the final result. Similarly, a cancellation of $1 / M_{V}^{2}$ enhanced terms in off-diagonal rates

1 The only minor discrepancies that we find are related to $P$-wave contributions: our results for ${ }^{3} P_{1}$-wave $\chi_{e_{1}}^{0} \chi_{e_{1}}^{0} \rightarrow H^{+} H^{-}$annihilations correspond to a factor 2 instead of a factor 4 in the second term of Eq. (A27b) in [22]. In the case of ${ }^{3} P_{0}$-wave $\chi_{e_{1}}^{0} \chi_{e_{1}}^{0} \rightarrow f \bar{f}$ reactions, our results correspond to a factor $\sqrt{2 / 3}$ instead of a factor $\sqrt{6}$ in the second term in the first line of Eq. (A29b) in [22].

${ }^{2}$ The authors of Ref. [14] also provided analytic results for exclusive leading-order ${ }^{1} S_{0}$-wave annihilation reactions for both the cases of a wino-like and a Higgsino-like neutralino LSP scenario in a previous work [13]. We agree with the results for all diagonal $\chi_{e_{1}} \chi_{e_{2}} \rightarrow \chi_{e_{1}} \chi_{e_{2}}$ reactions. A typo in the off-diagonal terms in Eq. (28) of [13] was fixed in 14, and the latter agrees with our findings. In the Higgsino-like scenario, we get differing expressions for off-diagonal $\chi_{i}^{0} \chi_{i}^{0} \rightarrow \chi_{1}^{-} \chi_{1}^{+}$and $\chi_{1}^{-} \chi_{1}^{+} \rightarrow \chi_{i}^{0} \chi_{i}^{0}$ reactions in the case of $W^{+} W^{-}$and $Z Z$ final states for both $i=1,2$ : our results are a factor 4 and a factor 2 larger, respectively, than the corresponding expressions presented in [13]. 
with one massive vector boson in the final state has to take place. However, for these cancellations to occur, one has to also expand the SUSY mixing matrices systematically in the gauge boson masses $M_{V}$. In the same way, the mass differences between the incoming and outgoing particles have to be expanded in $M_{V}$ and in the differences of soft SUSY breaking parameters $M_{1}, M_{2}, \mu$, if these differences are small. The latter expansions must be done differently depending on how many neutralinos and charginos are (nearly) mass-degenerate. The presentation of the results computed with unitary gauge then has to distinguish among many cases and also consider diagonal and offdiagonal terms separately, since for the diagonal terms it is desirable to keep the full mass dependence as well as unexpanded mixing matrices. We thus find it more convenient to use Feynman gauge for the calculation of the off-diagonal reactions, which allows to keep the coupling matrices unexpanded and a more concise presentation of the results. The price for this is that one must compute a large number of unphysical final states containing pseudo-Goldstone Higgs and ghost particles, see Tab. 3 .

\subsection{Off-diagonal terms}

Our framework aims to describe the annihilation of a pair of non-relativistic charginos or neutralinos $\left(\chi_{i} \chi_{j}\right)$ into SM and light Higgs particles pairs $\left(X_{A} X_{B}\right)$ including potential interactions between all nearly mass-degenerate $\chi \chi$ states, that can produce a Sommerfeld enhancement of the rates. A contribution to these enhanced annihilation rates is given by the imaginary part of the amplitude for a process of the type,

$$
\chi_{i} \chi_{j} \rightarrow \ldots \rightarrow \chi_{e_{1}} \chi_{e_{2}} \rightarrow X_{A} X_{B} \rightarrow \chi_{e_{4}} \chi_{e_{3}} \rightarrow \ldots \rightarrow \chi_{i} \chi_{j}
$$

where the intermediate states involved in the short-distance annihilation, $\chi_{e_{1}} \chi_{e_{2}}$ and $\chi_{e_{4}} \chi_{e_{3}}$, can be different (off-diagonal annihilation terms), compare to Fig. 1 for a figurative illustration. In a recent work [18, a general formalism which also aims to compute the Sommerfeld-enhanced annihilation rates for a coupled system of neutralino and chargino pairs, has been presented which, however, does not implement the possibility of off-diagonal transitions in the hard part of the annihilation process. We show in this section that the off-diagonal terms can indeed be relevant, and should be accounted for in the calculation of the Sommerfeld enhanced rates.

Naively, if the final state $X_{A} X_{B}$ is allowed for both $\chi_{e_{1}} \chi_{e_{2}}$ and $\chi_{e_{4}} \chi_{e_{3}}$, given one particular partial-wave configuration of the two-body systems, the off-diagonal absorptive amplitude can be of the same size as the diagonal absorptive amplitude, i.e.

$$
\begin{gathered}
\int\left[\mathrm{dPS}_{A B}\right] \mathcal{A}\left(\chi_{e_{1}} \chi_{e_{2}} \rightarrow X_{A} X_{B}\right) \times \mathcal{A}\left(\chi_{e_{4}} \chi_{e_{3}} \rightarrow X_{A} X_{B}\right)^{*} \\
\sim \int\left[\mathrm{dPS}_{A B}\right]\left|\mathcal{A}\left(\chi_{e_{1}} \chi_{e_{2}} \rightarrow X_{A} X_{B}\right)\right|^{2}
\end{gathered}
$$

since the phase-space integration involves very similar kinematics. An example of the latter is given by the annihilation rates of wino-like neutralino dark matter, where the 
wino-like neutralino $\left(\chi_{1}^{0}\right)$ is highly degenerate with its charged $S U(2)_{L}$ partners $\left(\chi_{1}^{ \pm}\right)$. In such scenario the spin-0 $\chi_{1}^{0} \chi_{1}^{0}$ system mixes with the $\chi_{1}^{-} \chi_{1}^{+}$state through $W$-boson exchange. The inclusive annihilation rates that have to be fed into the calculation of the enhanced rates for the spin- $0 \chi_{1}^{0} \chi_{1}^{0}$ channel in the wino limit read

$$
\begin{gathered}
{\left.\left[\mathcal{A}\left(\chi_{1}^{0} \chi_{1}^{0} \rightarrow \chi_{1}^{0} \chi_{1}^{0}\right)\left({ }^{1} S_{0}\right)\right]\right|_{\mathrm{abs}}=2 f_{\{11\}\{11\}}^{00 \rightarrow 00}\left({ }^{1} S_{0}\right)=\frac{4 \pi \alpha_{2}^{2}}{m_{\chi^{0}}^{2}},} \\
{\left.\left[\mathcal{A}\left(\chi_{1}^{-} \chi_{1}^{+} \rightarrow \chi_{1}^{-} \chi_{1}^{+}\right)\left({ }^{1} S_{0}\right)\right]\right|_{\mathrm{abs}}=2 f_{\{11\}\{11\}}^{-+\rightarrow-+}\left({ }^{1} S_{0}\right)=\frac{3 \pi \alpha_{2}^{2}}{m_{\chi^{0}}^{2}}} \\
{\left.\left[\mathcal{A}\left(\chi_{1}^{0} \chi_{1}^{0} \rightarrow \chi_{1}^{-} \chi_{1}^{+}\right)\left({ }^{1} S_{0}\right)\right]\right|_{\mathrm{abs}}=2 f_{\{11\}\{11\}}^{00 \rightarrow-+}\left({ }^{1} S_{0}\right)=\frac{2 \pi \alpha_{2}^{2}}{m_{\chi^{0}}^{2}}}
\end{gathered}
$$

where $\alpha_{2}=g_{2}^{2} / 4 \pi, g_{2}$ denotes the $S U(2)_{L}$ gauge coupling, and all gauge boson and Higgs-particle masses are treated as massless. We see explicitly that the off-diagonal term (36) is of the same order as the diagonal reactions (34 35).

In order to stress the importance of the off-diagonal annihilation terms, we have computed the thermally averaged effective annihilation cross section, $\left\langle\sigma_{\text {eff }} v\right\rangle$, which enters the Boltzmann equation for the calculation of the dark matter yield, for the same wino-like scenario used for the checks with MADGRAPH presented in Sec. 3.1, and compared to the results obtained when the off-diagonal terms are switched off by hand. For the necessary formulas to compute $\left\langle\sigma_{\text {eff }} v\right\rangle$, including co-annihilation effects, we refer the reader to [27, 28]. The annihilation rates have been calculated using (27) with Wilson coefficients multiplied by the appropriate Sommerfeld factors computed solving the coupled-channel Schrödinger equation for each partial-wave. The details about the calculation of the Sommerfeld enhancement factors from the long-range interactions will be given in a future publication [24].

We observe from Fig. 4 that removing the off-diagonal annihilation terms decreases the thermally averaged cross section by a factor larger than 1.5 at small temperatures. The corresponding thermal relic abundance of the dark matter in the present Universe, $\Omega_{\mathrm{DM}} h^{2}$, obtained by numerical integration of the Boltzmann equation, gets then increased by approximately $20 \%$, if the off-diagonal reactions are neglected. The latter represents thus a sizeable effect which has to be accounted for in such a scenario.

\section{Summary}

The calculation of the thermal relic abundance of the lightest neutralino as a promising dark matter candidate within the MSSM places strong bounds on the MSSM parameter space, assuming that the observed cosmic dark matter has particle nature and is composed solely of the neutralino LSP. Given the expected future experimental accuracy of the measurement of the cosmic dark matter abundance observed today, radiative corrections to the pure tree-level annihilation cross section, entering the relic abundance 


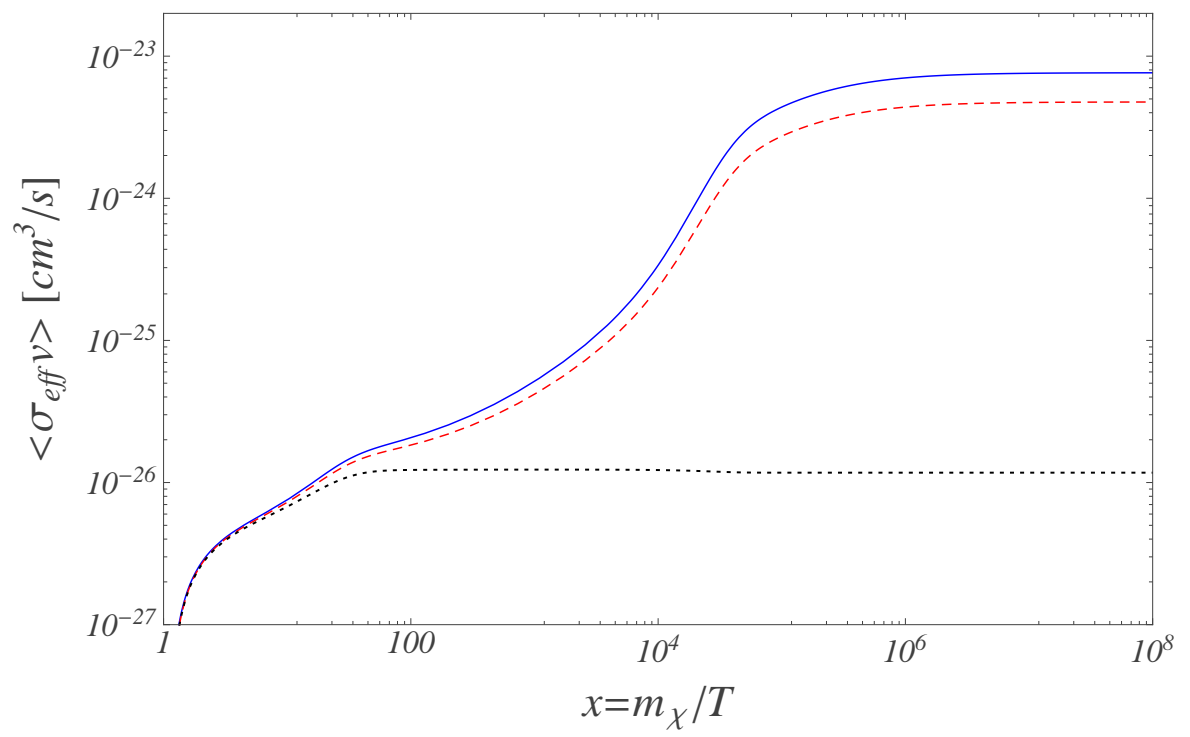

Figure 4: Thermally averaged effective annihilation cross section as a function of $x=$ $m_{\chi} / T$ with fixed $m_{\chi}=2748.92 \mathrm{GeV}$, including the Sommerfeld effect (solid blue line). The same quantity computed with the off-diagonal perturbative annihilation rates set to zero is depicted by the dashed red line. The perturbative result is also shown as a dotted line for comparison. The decrease of $\left\langle\sigma_{\text {eff }} v\right\rangle$ towards $x \sim 1$ is due to large negative $\mathcal{O}\left(\vec{p}^{2}\right)$ terms in the $\chi \chi$ annihilation rates, which are unphysical because the non-relativistic expansion (27) for the annihilation rate becomes unreliable for large temperatures.

calculation as a central ingredient, should eventually be taken into account. The inclusion of 1-loop corrections to the annihilation cross section as well as the systematic treatment of Sommerfeld enhancements, has recently been a field of elaborate studies in the literature. Similarly, in the context of dark matter annihilation processes in the present Universe relevant in indirect detection, the above types of radiative corrections to the neutralino pair-annihilation cross section have been studied extensively.

In this paper we take advantage of the non-relativistic nature of the annihilating neutralinos in the present Universe as well as during thermal dark matter decoupling in the early Universe, which introduces a clear separation of energy scales in all annihilation processes of interest. The latter property allows us to set up an effective field theory (the NRMSSM) of non-relativistic neutralinos and charginos, that provides an appropriate setup for a systematic investigation of radiative corrections to neutralino LSP pair annihilation processes both in the present and the early Universe, taking coannihilations with nearly mass-degenerate neutralinos and charginos into account. As a first step in the explicit construction of the NRMSSM we have derived fully analytic formulas for the absorptive part of the Wilson coefficients of four-fermion operators in the effective theory pertaining to $S$-wave annihilation, that encode the hard annihilation rates of $\chi_{e_{1}} \chi_{e_{2}} \rightarrow X_{A} X_{B}$ processes (see (6) and Tab. 2). Our results separately include 
leading-order ${ }^{1} S_{0^{-}}$and ${ }^{3} S_{1}$-wave as well as all $P$-wave and next-to-next-to-leading order $S$-wave Wilson coefficients and apply to general neutralino and chargino states in the MSSM. Flavour off-diagonal sfermion generation mixing can be covered, and we keep the full mass dependence of all SM and Higgs particles. Analytic results for the absorptive part of leading order $S$-wave Wilson coefficients are presented in the appendix. Results for $P$-wave and next-to-next-to-leading order $S$-wave coefficients will be given in a future publication [23]. By taking into account charge-neutral annihilation processes of a chargino pair as well as singly charged and doubly charged annihilation reactions of non-relativistic neutralinos and charginos, we extend the analytic results for partial wave decomposed neutralino LSP pair-annihilation cross sections given in the literature [22].

We have shown that the non-relativistic expansion to $\mathcal{O}\left(v^{2}\right)$ produces accurate results up to $v_{\text {rel }} \sim 0.6$, which is sufficient for relic density computations, and certainly for dark matter annihilation in the present Universe. Our analytic results may therefore substitute for time-consuming numerical computations.

Our aim is to apply the effective field theory formalism to the calculation of Sommerfeld-enhanced (co-)annihilation cross sections in the neutralino relic abundance calculation. As scattering prior to the annihilation process can lead to transitions from an incoming particle pair to another nearly mass-degenerate neutralino or chargino twoparticle state, a proper treatment of the Sommerfeld effect requires the knowledge of the absorptive part of off-diagonal annihilation rates, $\chi_{e_{1}} \chi_{e_{2}} \rightarrow X_{A} X_{B} \rightarrow \chi_{e_{4}} \chi_{e_{3}}$, for all possible SM and Higgs two-particle states $X_{A} X_{B}$ (see Fig. 1). To the best of our knowledge we present for the first time analytic results that allow for a systematic treatment of all these off-diagonal rates in Sommerfeld-enhanced (co-)annihilation reactions for general

masses and composition of the $\chi_{e_{i}}$ particles. The implications of these results for MSSM relic density calculations will be studied in a forth-coming publication [24].

\section{Note added}

The present arXiv version replaces an incorrect version of Figure 4 and fixes some typos which are also present in the journal publication. For an explicit list of errata see the JHEP erratum [34].

\section{Acknowledgements}

We would like to thank N. Baro for discussions and some cross checks of the tree-level annihilation cross sections with the private code Sloops [11, 12, 29]. The work of M.B. was supported in part by the DFG Sonderforschungsbereich/Transregio 9 "Computergestützte Theoretische Teilchenphysik". C.H. greatly acknowledges the support by the "Deutsche Telekom Stiftung". The work of P. R. is partially supported by MEC (Spain) under grants FPA2007-60323 and FPA2011-23778 and by the Spanish Consolider-Ingenio 2010 Programme CPAN (CSD2007-00042). Feynman diagrams have been drawn with the packages AxODRAW [30] and JAXODRAW [31]. 


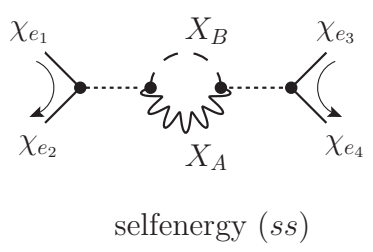

Figure 5: Generic selfenergy-diagram in $\chi \chi \rightarrow X_{A} X_{B} \rightarrow \chi \chi$ reactions. Particles $X_{A}$ and $X_{B}$ represent any two-body final state of SM and Higgs particles, which can be produced on-shell in $\chi \chi \rightarrow X_{A} X_{B}$ annihilations.

\section{A Absorptive parts of Wilson coefficients of dim- ension-6 operators in $\delta \mathcal{L}_{\text {ann }}$}

We present the leading order contributions to the absorptive part, $\hat{f} \chi \chi \rightarrow \chi \chi\left({ }^{2 s+1} L_{J}\right)$, of the Wilson coefficients that correspond to the local four-fermion operators given in Tab. 2 , The $\hat{f} \chi_{e_{1}} \chi_{e_{2}} \rightarrow \chi_{e_{4}} \chi_{e_{3}}\left({ }^{2 s+1} L_{J}\right)$ encode the absorptive part of hard $2 \rightarrow 2$ scattering reactions of an incoming particle pair $\chi_{e_{1}} \chi_{e_{2}}$ of non-relativistic charginos or neutralinos in a given ${ }^{2 s+1} L_{J}$ partial-wave state into an outgoing non-relativistic $\chi_{e_{4}} \chi_{e_{3}}$-pair in the same partialwave configuration. They allow to reproduce the inclusive tree-level center-of-mass frame annihilation cross sections of a non-relativistic $\chi_{i} \chi_{j}$-pail ${ }^{3}$ into SM and light Higgs twobody final states $X_{A} X_{B}$, expanded in the relative velocity of the annihilating particle pair. The general case includes off-diagonal processes $\chi_{e_{1}} \chi_{e_{2}} \rightarrow X_{A} X_{B} \rightarrow \chi_{e_{4}} \chi_{e_{3}}$ with $\chi_{e_{1}} \chi_{e_{2}} \neq \chi_{e_{4}} \chi_{e_{3}}$, for all pairs of non-relativistic neutralinos and charginos. Since the $\hat{f} \chi \chi \rightarrow \chi \chi\left({ }^{2 s+1} L_{J}\right)$ are infrared-safe at leading order, we are able to give analytic results for the individual contributions $\hat{f} \chi \chi \rightarrow X_{A} X_{B} \rightarrow \chi \chi\left({ }^{2 s+1} L_{J}\right)$ pertaining to an exclusive final state $X_{A} X_{B}$.

\section{A.1 Notation and definitions}

Recall that the calculation is performed in Feynman gauge. Hence the two-particle final states $X_{A} X_{B}$ that we account for can be classified to be of vector-vector $(V V)$, vectorscalar $(V S)$, scalar-scalar $(S S)$, fermion-antifermion $(f f)$ or ghost-anti-ghost $(\eta \bar{\eta})$ type. They are listed in Tab. 3. The determination of the absorptive part of the Wilson coefficients for the processes $\chi_{e_{1}} \chi_{e_{2}} \rightarrow X_{A} X_{B} \rightarrow \chi_{e_{4}} \chi_{e_{3}}$ requires the calculation of a large number of Feynman diagrams. To be able to present the results in an efficient manner it is convenient to make use of the classification in $V V_{-}, V S-, S S-, f f-$ and $\eta \bar{\eta}_{-}$ type $X_{A} X_{B}$ particle states and to further subdivide the contributing diagrams according to their topology. In each of the classes under consideration there arise generic 1-loop amplitudes with selfenergy-, triangle- and box-topology shown in Figs. 56 8 . The generic selfenergy-diagram as well as the four generic triangle- and box-diagrams cover

\footnotetext{
${ }^{3}$ The covered $\chi_{i} \chi_{j}$-states have been collected in Tab. 1.
} 


\begin{tabular}{|c|c|c|c|c|c|}
\hline$\chi \chi \rightarrow \chi \chi$ & $V V$ & $V S$ & $S S$ & $f f$ & $\overline{\eta \bar{\eta}}$ \\
\hline $\begin{array}{c}\chi^{0} \chi^{0} \rightarrow \chi^{0} \chi^{0} \\
\chi^{-} \chi^{+} \rightarrow \chi^{-} \chi^{+} \\
\chi^{0} \chi^{0} \rightarrow \chi^{-} \chi^{+} \\
\chi^{-} \chi^{+} \rightarrow \chi^{0} \chi^{0}\end{array}$ & $\begin{array}{c}W^{+} W^{-} \\
Z Z \\
\gamma \gamma, Z \gamma\end{array}$ & $\begin{array}{c}Z h^{0}, Z H^{0}, \\
\gamma h^{0}, \gamma H^{0}, \\
Z G^{0}, Z A^{0}, \\
\gamma G^{0}, \gamma A^{0}, \\
W^{+} G^{-}, W^{+} H^{-}, \\
W^{-} G^{+}, W^{-} H^{+}\end{array}$ & $\begin{array}{c}h^{0} h^{0}, h^{0} H^{0}, H^{0} H^{0}, \\
G^{0} h^{0}, A^{0} h^{0} \\
G^{0} H^{0}, A^{0} H^{0} \\
G^{0} G^{0}, G^{0} A^{0}, A^{0} A^{0} \\
G^{+} G^{-}, G^{+} H^{-} \\
H^{+} G^{-}, H^{+} H^{-}\end{array}$ & $\begin{array}{l}u^{J} \bar{u}^{I}, \\
d^{J} \bar{d}^{I}, \\
e^{J} \bar{e}^{I} \\
\nu^{J} \bar{\nu}^{I}\end{array}$ & $\begin{array}{c}\eta^{+} \bar{\eta}^{+} \\
\eta^{-} \bar{\eta}^{-} \\
\eta^{Z} \bar{\eta}^{Z}\end{array}$ \\
\hline$\chi^{0} \chi^{+} \rightarrow \chi^{0} \chi^{+}$ & $\begin{array}{l}W^{+} Z \\
W^{+} \gamma\end{array}$ & $\begin{array}{c}Z G^{+}, \gamma G^{+}, \\
Z H^{+}, \gamma H^{+}, \\
W^{+} h^{0}, W^{+} H^{0}, \\
W^{+} G^{0}, W^{+} A^{0}\end{array}$ & $\begin{array}{l}G^{+} h^{0}, G^{+} H^{0}, \\
H^{+} h^{0}, H^{+} H^{0}, \\
G^{+} G^{0}, G^{+} A^{0}, \\
H^{+} G^{0}, H^{+} A^{0}\end{array}$ & $\begin{array}{l}u^{J} \bar{d}^{I}, \\
\nu^{J} \bar{e}^{I}\end{array}$ & $\begin{array}{l}\eta^{+} \bar{\eta}^{Z} \\
\eta^{Z} \bar{\eta}^{-} \\
\eta^{+} \bar{\eta}^{F} \\
\eta^{F} \bar{\eta}^{-}\end{array}$ \\
\hline$\chi^{+} \chi^{+} \rightarrow \chi^{+} \chi^{+}$ & $W^{+} W^{+}$ & $\begin{array}{l}W^{+} G^{+}, \\
W^{+} H^{+}\end{array}$ & $\begin{array}{l}G^{+} G^{+} \\
G^{+} H^{+} \\
H^{+} H^{+}\end{array}$ & & \\
\hline
\end{tabular}

Table 3: Particle pairs $X_{A} X_{B}$ in $\chi \chi \rightarrow X_{A} X_{B} \rightarrow \chi \chi$ scattering reactions (abbreviated as $\chi \chi \rightarrow \chi \chi)$, that we account for in the calculation of the absorptive part of the Wilson coefficients, classified according to their type: $V V, V S, S S, f f$ and $\eta \bar{\eta}$. Negatively charged processes, corresponding to the charge-conjugates of the singly or doubly positively charged reactions above are not explicitly written.

all possible kinematic configurations that can arise in a $\chi_{e_{1}} \chi_{e_{2}} \rightarrow X_{A} X_{B} \rightarrow \chi_{e_{4}} \chi_{e_{3}}$ 1-loop amplitude. Note that we have assigned specific directions for the fermion flow in

\footnotetext{
${ }^{4}$ The case of four different triangle- and four different box-diagrams in Fig. 6 and Fig. 7 applies to non-identical particles $X_{A} \neq X_{B}$. For identical particles $X_{A}=X_{B}$, triangle (box) 1 and 3 as well as triangle (box) 2 and 4 coincide. In this case only one of the identical diagrams must be taken into account to compute the corresponding $\hat{f} \chi \chi \rightarrow X_{A} X_{A} \rightarrow \chi \chi$ coefficients. This rule incorporates the symmetry factor of $1 / 2$ in the cross-section for identical final-state particles, that one would take into account in the conventional calculation of the tree-level $\chi_{e_{1}} \chi_{e_{2}} \rightarrow X_{A} X_{A}$ annihilation rate.
}

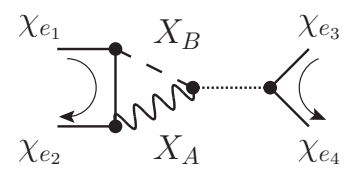

triangle $1\left(t_{1} s\right)$

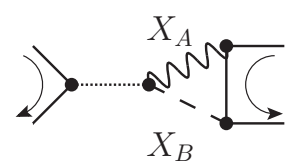

triangle $2\left(s t_{1}\right)$

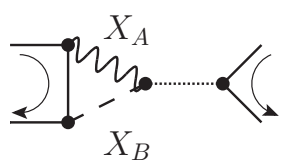

triangle $3\left(t_{2} s\right)$

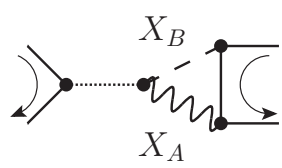

triangle $4\left(s t_{2}\right)$

Figure 6: Generic triangle-diagrams in $\chi \chi \rightarrow X_{A} X_{B} \rightarrow \chi \chi$ reactions. 


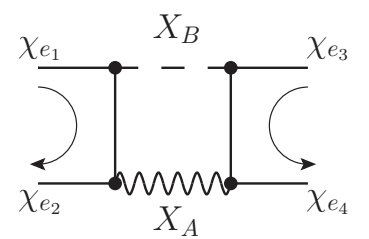

box $1\left(t_{1} t_{2}\right)$

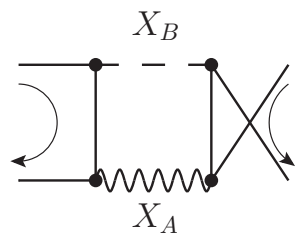

box $2\left(t_{1} t_{1}\right)$

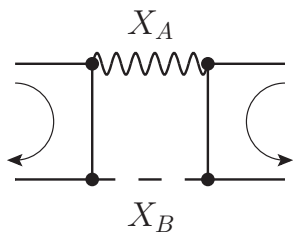

box $3\left(t_{2} t_{1}\right)$

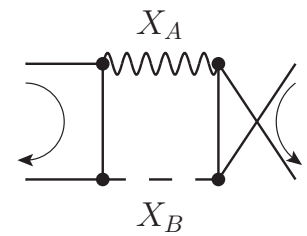

box $4\left(t_{2} t_{2}\right)$

Figure 7: Generic box-diagrams in $\chi \chi \rightarrow X_{A} X_{B} \rightarrow \chi \chi$ reactions.

each diagram in Figs. 518, indicated by the arrows, as it is convenient in the context of calculations involving both Dirac and Majorana fermions, following the Feynman rules for fermion-number violating interactions set out in [32]. The depicted fermion flows establish our convention to arrange the external fermion states $\chi_{e_{i}}, i=1, \ldots, 4$ in descending order, see Tab. 2.

We calculate analytically the absorptive part of any of the contributing selfenergy-, triangle- and box-amplitudes, subject to our convention for the fermion flows. Thereby we consider generic external Majorana fermions, generic t- and u-channel exchanged Majorana fermions or sfermions, generic $X_{A} X_{B}$ states of type $V V, V S, S S, f f$ and $\eta \bar{\eta}$, and hence use generic 'place-holder' coupling factors at each vertex. This allows us to determine the generic form of those terms in the contributions to the $\hat{f} \chi \chi \rightarrow X_{A} X_{B} \rightarrow \chi \chi\left({ }^{2 s+1} L_{J}\right)$, that are associated with the kinematics of the $\chi \chi \rightarrow X_{A} X_{B} \rightarrow \chi \chi$ reaction, where each of these kinematic terms multiplies a certain combination of the place-holder coupling factors. In particular, these kinematic contributions are generic in the sense that they apply to both the cases of external and internal Majorana and Dirac fermions.

A specific diagram's contribution to the absorptive part of a particular $\chi_{e_{1}} \chi_{e_{2}} \rightarrow$ $X_{A} X_{B} \rightarrow \chi_{e_{4}} \chi_{e_{3}}$ MSSM 1-loop process is obtained by replacing the generic placeholder coupling factors with their actual expressions in the above described generic Majorana fermion $2 \rightarrow 2$ scattering reactions. Note that by choosing these coupling factors properly, all $\chi_{e_{1}} \chi_{e_{2}} \rightarrow X_{A} X_{B} \rightarrow \chi_{e_{4}} \chi_{e_{3}}$ processes with external and internal

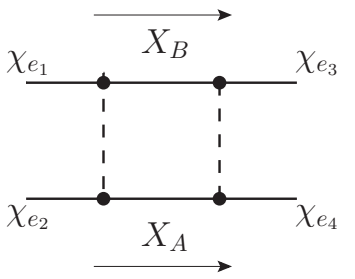

box $1\left(t_{1} t_{2}\right)$

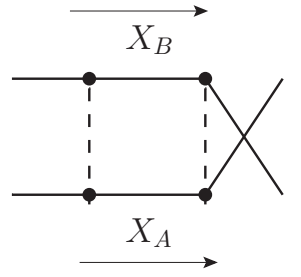

box $2\left(t_{1} t_{1}\right)$

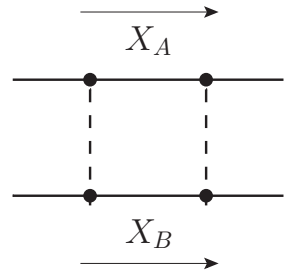

$\operatorname{box} 3\left(t_{2} t_{1}\right)$

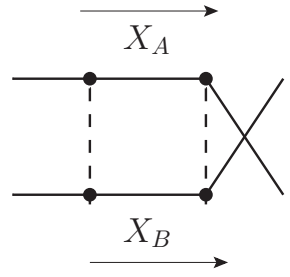

box $4\left(t_{2} t_{2}\right)$

Figure 8: Generic box-diagrams in $\chi \chi \rightarrow X_{A} X_{B} \rightarrow \chi \chi$ reactions, with $X_{A} X_{B}$ a pair of SM fermions. 
Majorana or Dirac fermions can be covered, although the kinematic contributions are calculated referring to the generic Majorana fermion $2 \rightarrow 2$ scattering reaction. Hence, the absorptive part of the Wilson coefficient, which encodes the absorptive part of a $\chi_{e_{1}} \chi_{e_{2}} \rightarrow X_{A} X_{B} \rightarrow \chi_{e_{4}} \chi_{e_{3}}$ scattering reaction, with the incoming and outgoing twoparticle states in a ${ }^{2 s+1} L_{J}$ partial-wave configuration, can be written as

$$
\begin{aligned}
& \hat{f}_{\left\{e_{1} e_{2}\right\}\left\{e_{4} e_{3}\right\}}^{\chi_{e_{1}} \chi_{e_{2}} \rightarrow X_{A} X_{B} \rightarrow \chi_{e_{4}} \chi_{e_{3}}\left({ }^{2 s+1} L_{J}\right)} \\
& =\frac{\pi \alpha_{2}^{2}}{M^{2}}\left(\sum_{n} \sum_{i_{1}, i_{2}} b_{n, i_{1} i_{2}}^{\chi_{e_{1}} \chi_{e_{2}} \rightarrow X_{A} X_{B} \rightarrow \chi_{e_{4}} \chi_{e_{3}}} B_{n, i_{1} i_{2}}^{X_{A} X_{B}}\left({ }^{2 s+1} L_{J}\right)\right. \\
& +\sum_{\alpha=1}^{4} \sum_{n} \sum_{i_{1}, i_{2}} c_{n, i_{1} i_{2}}^{(\alpha) \chi_{e_{1}} \chi_{e_{2}} \rightarrow X_{A} X_{B} \rightarrow \chi_{e_{4}} \chi_{e_{3}}} C_{n, i_{1} i_{2}}^{(\alpha) X_{A} X_{B}}\left({ }^{2 s+1} L_{J}\right) \\
& \left.+\sum_{\alpha=1}^{4} \sum_{n} \sum_{i_{1}, i_{2}} d_{n, i_{1} i_{2}}^{(\alpha) \chi_{e_{1}} \chi_{e_{2}} \rightarrow X_{A} X_{B} \rightarrow \chi_{e_{4}} \chi_{e_{3}}} D_{n, i_{1} i_{2}}^{(\alpha) X_{A} X_{B}}\left({ }^{2 s+1} L_{J}\right)\right) .
\end{aligned}
$$

Here $\alpha_{2}=g_{2}^{2} / 4 \pi$, where $g_{2}$ denotes the $S U(2)_{L}$ gauge coupling. The sums in the first line on the right-hand side of (37) collect all contributions from selfenergy-amplitudes. Similarly, the second (third) line gives the triangle- (box-) amplitudes' contributions. We use the index $\alpha$ to enumerate expressions related to the four different triangle- and boxamplitudes 5 according to the labelling of the diagrams in Figs. 66 8, Further, we indicate the kinematic factors of the generic $2 \rightarrow 2$ Majorana fermion scattering amplitudes within a given class and topology with capital letters $\left(B_{n, i_{1} i_{2}}, C_{n, i_{1} i_{2}}^{(\alpha)}, D_{n, i_{1} i_{2}}^{(\alpha)}\right)$. These are the quantities that include the kinematics of the process and hence encode the ${ }^{2 s+1} L_{J}$ partial-wave specific information. The process-specific coupling factors that multiply the kinematic factors are denoted with lowercase letters $\left(b_{n, i_{1} i_{2}}, c_{n, i_{1} i_{2}}^{(\alpha)}, d_{n, i_{1} i_{2}}^{(\alpha)}\right)$. Depending on the type of the particles $X_{A}$ and $X_{B}$ as well as the topology, there is a fixed number of different coupling-factor expressions that can occur, together with the corresponding kinematic factors. The different contributions are enumerated with the index $n$ in (37) above. Finally, in each of the processes there is a certain set of particle species that can be exchanged in the $s$ - or the $t$-channels of the contributing amplitudes. These are labelled with the indices $i_{1}$ and $i_{2}$.

The generic structure of the Wilson coefficients in (37) suggests to give the coupling factors and the kinematic factors separately. A recipe for the construction of the coupling factors $b_{n, i_{1} i_{2}}, c_{n, i_{1} i_{2}}^{(\alpha)}, d_{n, i_{1} i_{2}}^{(\alpha)}$ in any of the covered reactions is given in Sec. A.2. Analytic results for the kinematic factors $B_{n, i_{1} i_{2}}, C_{n, i_{1} i_{2}}^{(\alpha)}, D_{n, i_{1} i_{2}}^{(\alpha)}$ for the leading-order ${ }^{1} S_{0}$ and ${ }^{3} S_{1}$ partial-wave configurations can be found in Sec. A.3. These expressions depend on the masses of the external and internal particles in a particular $\chi_{e_{1}} \chi_{e_{2}} \rightarrow X_{A} X_{B} \rightarrow \chi_{e_{4}} \chi_{e_{3}}$ process. However, the kinematic factors are generic in the sense that their form is

\footnotetext{
${ }^{5}$ For identical particles $X_{A}=X_{B}$ the index $\alpha$ has to be taken from 1 to 2 only, see footnote 4 .
} 
the same for all possible external two-body states $\chi_{e_{1}} \chi_{e_{2}}$ and $\chi_{e_{3}} \chi_{e_{4}}$ of neutralinos or charginos and all $X_{A} X_{B}$ particles within one of the classes $V V, V S, S S, f f$ or $\eta \bar{\eta}$.

The coupling and kinematic factors will depend on the supersymmetric particles' mixing matrices and masses, respectively. We adopt the same notation as in [33] and hence introduce the chargino and neutralino mixing matrices $Z_{ \pm}$and $Z_{N}$ defined via

$$
\begin{aligned}
& Z_{-}^{T} M_{\chi^{ \pm}} Z_{+}=\left(\begin{array}{ll}
m_{\chi_{1}^{+}} & \\
& m_{\chi_{2}^{+}}
\end{array}\right), \\
& Z_{N}^{T} M_{\chi^{0}} Z_{N}=\left(\begin{array}{llll}
m_{\chi_{1}^{0}} & & & \\
& m_{\chi_{2}^{0}} & & \\
& & m_{\chi_{3}^{0}} & \\
& & & m_{\chi_{4}^{0}}
\end{array}\right) \text {, }
\end{aligned}
$$

where $M_{\chi^{ \pm}}$and $M_{\chi^{0}}$ denote the chargino and neutralino mass matrices, respectively (for details regarding the mass matrix expressions refer to [33]). $m_{\chi_{j}^{+}}, j=1,2$, and $m_{\chi_{i}^{0}}, i=$ $1, \ldots 4$ indicate the masses in the mass eigenstate basis of charginos and neutralinos.

In order to properly apply the formulas for coupling and kinematic factors in Sec. A.2 and Sec. A.3 given a specific MSSM spectrum, it is important to note that the NRMSSM and hence the analytic expressions for the Wilson coefficients explicitly rely on the positivity of all mass parameters. This derives from the fact that the NRMSSM Lagrangian is obtained by extracting the high-energy fluctuations (of the order of the particle mass) from the relativistic fields, which yields the non-relativistic kinetic term $\mathcal{L}_{\text {kin }}$ shown in (3). For species other than the LSP, the procedure leads to the mass-difference terms $\left(m_{i}-m_{\mathrm{LSP}}\right)$ in (3). If any of the $m_{i}$ in $\mathcal{L}_{\text {kin. }}$ is negative, then the corresponding mass difference counts as $\mathcal{O}\left(m_{\mathrm{LSP}}\right)$, an indication that the parametrization used to relate the relativistic and non-relativistic fields for that particle species is not the appropriate one. The simplest way to obtain the NRMSSM Lagrangian in case that the mass $m_{\chi_{e_{i}}}$ of one or several of the external $\chi_{e_{i}}$ particles happens to be negative for a given MSSM spectrum, is to perform a field redefinition of the corresponding MSSM fields that yields mass terms with positive mass parameters. Such a field redefinition affects the chargino and neutralino mixing matrices, which are mapped in the following way:

$$
\begin{aligned}
& Z_{ \pm} \rightarrow \widetilde{Z}_{ \pm}=Z_{ \pm} \cdot\left(\begin{array}{ll}
\sqrt{\operatorname{sgn}\left(m_{\chi_{1}^{+}}\right)} & \\
& \sqrt{\operatorname{sgn}\left(m_{\chi_{2}^{+}}\right)}
\end{array}\right)
\end{aligned}
$$

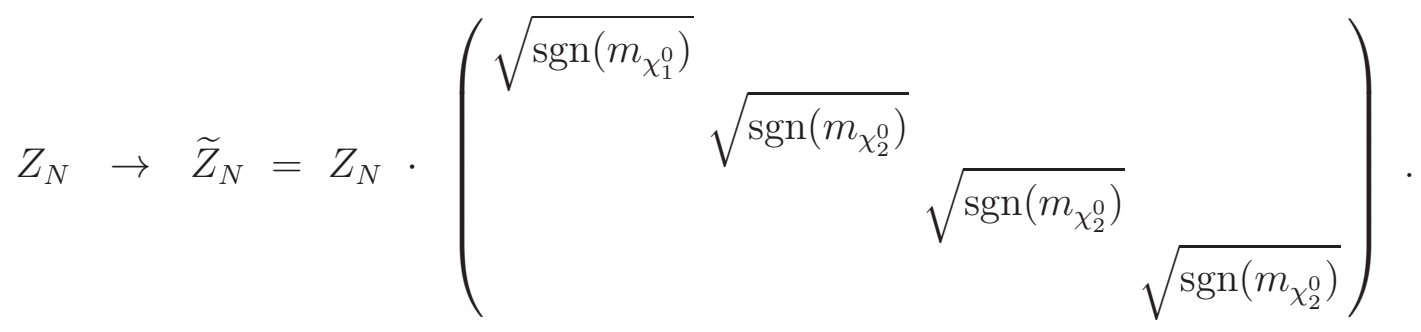




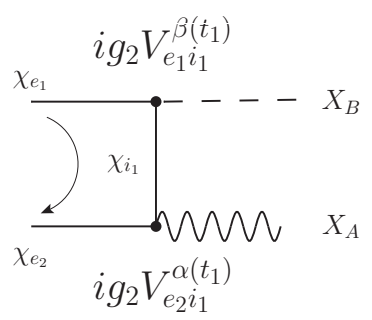

diagram $t_{1}$

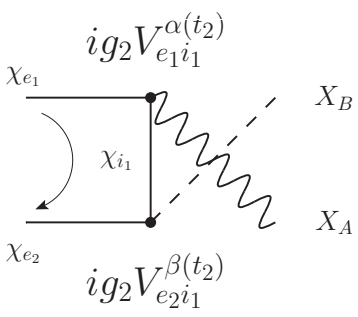

diagram $t_{2}$

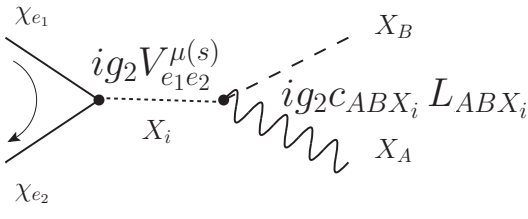

diagram $s$

Figure 9: Generic tree-level amplitudes in $\chi \chi \rightarrow X_{A} X_{B}$ annihilations, referring to $V V, V S$ and $S S$-type final state particles $X_{A} X_{B}$. The generic form of $s$-channel exchange diagrams for $X_{A} X_{B}=\eta \bar{\eta}$ final states agrees with the $s$-channel diagram above. The vertex-factors $V_{e i}^{\rho(d)}$ are defined as $V_{e i}^{\rho(d)}=\gamma^{\rho}\left(r_{e i}^{(d)}+q_{e i}^{(d)} \gamma_{5}\right)$, if attached to a three-point vertex with a gauge-boson (with Lorentz-index $\rho$ ), and $V_{e i}^{\rho(d)}=\left(r_{e i}^{(d)}+q_{e i}^{(d)} \gamma_{5}\right)$, if associated with a vertex that involves a scalar particle $X_{A}, X_{B}$ or $X_{i}$. Here the expression $r_{e i}^{(d)}\left(q_{e i}^{(d)}\right)$ either denotes a vector or scalar (an axial-vector or pseudo-scalar) type of coupling factor. For the definition of $c_{A B X_{i}}$ and the Lorentz structures $L_{A B X_{i}}$ we refer to Tab. 4 below.

(We define $\sqrt{-1}=i$.) The redefined mixing matrices $\widetilde{Z}_{ \pm}$and $\widetilde{Z}_{N}$ as well as the corresponding positive mass parameters for all MSSM neutralino and chargino fields should be used within the expressions given in Sec. A.2 and Sec. A.3.

\section{A.2 Coupling factors}

By construction, the absorptive part $\hat{f} \chi_{e_{1}} \chi_{e_{2}} \rightarrow X_{A} X_{B} \rightarrow \chi_{e_{4}} \chi_{e_{3}}$ of an individual Wilson co-

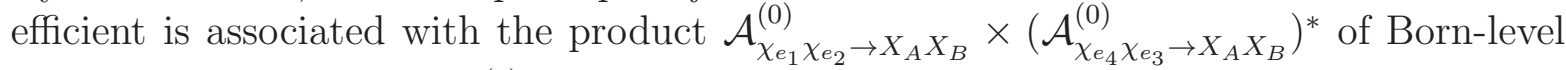
annihilation amplitudes $\mathcal{A}^{(0)}$ related to $\chi_{e_{i}} \chi_{e_{j}} \rightarrow X_{A} X_{B}$ reactions, integrated over the $X_{A} X_{B}$ two-particle phase space, see (16). Each of the tree-amplitudes $\mathcal{A}_{\chi \chi \rightarrow X_{A} X_{B}}^{(0)}$ receives contributions from diagrams with $t$-channel neutralino or chargino exchange as well as from diagrams with $s$-channel Higgs-particle or gauge-boson exchange, such as the generic diagrams shown in Fig. 9. In case of fermionic final states $X_{A} X_{B}$, instead of neutralino or chargino $t$-channel exchange, $t$-channel sfermion-exchange occurs, as depicted in Fig. 10, Note, that in Fig. 9 and Fig. 10 we again have established a specific fermion flow, which in particular coincides with the convention for the fermion flow associated with the incoming two particles in the 1-loop amplitudes in Figs. 58 .

A contribution to the amplitude $\mathcal{A}_{\chi \chi \rightarrow X_{A} X_{B}}^{(0)}$ involves a product of two coupling factors, coming from the two vertices in the tree-level diagrams. The generic form of these vertices is indicated in Fig. 9 and Fig. 10, It is especially convenient to write all vertex factors in any of the amplitudes contributing to the non-relativistic $\chi \chi \rightarrow X_{A} X_{B} \rightarrow \chi \chi$ scatteringprocesses as a combination of (axial-) vector or (pseudo-) scalar coupling factors, instead of using left- and right-handed couplings, as it is common in calculations related to the 


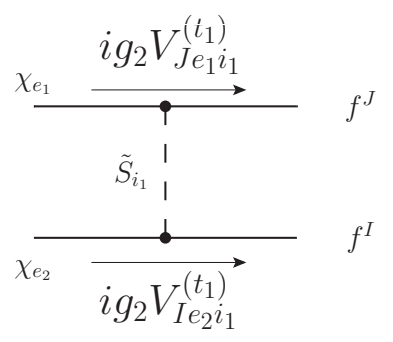

diagram $t_{1}$

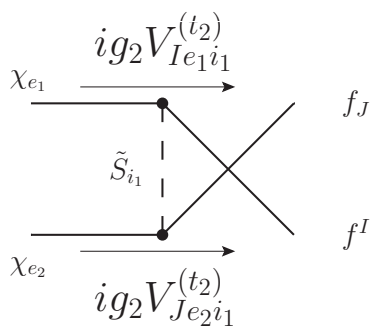

diagram $t_{2}$

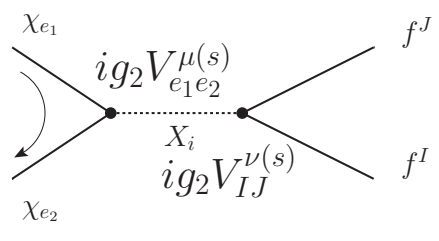

$\operatorname{diagram} s$

Figure 10: Generic tree-level amplitudes in $\chi \chi \rightarrow X_{A} X_{B}$ annihilations, with $X_{A} X_{B}=$ $f^{I} f^{J}$. For the definition of $V_{e i}^{\rho(d)}$ see Fig. 9. The generic vertex factor $V_{K e i}^{(d)}$ is defined as $V_{K e i}^{(d)}=r_{K e i}^{(d)}+q_{K e i}^{(d)} \gamma_{5}$, such that the $r_{K e i}^{(d)}\left(q_{K e i}^{(d)}\right)$ denote coupling factors of scalar (pseudo-scalar) type.

MSSM. The reason for that is, that in the non-relativistic limit, either the contributions to the annihilation amplitudes involving the axial-vector (pseudo-scalar) coupling will be suppressed with respect to the corresponding contributions related to the vector (scalar) coupling, or vice versa, such that the use of (axial-) vector and (pseudo-) scalar couplings allows for a clearer understanding of leading and suppressed contributions in the nonrelativistic scattering regime that we aim to study.

Each of the coupling factors $b_{n}, c_{n}^{(\alpha)}$ and $d_{n}^{(\alpha)}$ that occur in (37) is given by a product of two coupling factors, $r$ or $q$, arising in an individual diagram in $\mathcal{A}_{\chi_{e_{1}} \chi_{e_{2}} \rightarrow X_{A} X_{B}}^{(0)}$, and the complex conjugate of another such two-coupling factor product originating from $\mathcal{A}_{\chi_{e_{4}} \chi_{e_{3}} \rightarrow X_{A} X_{B}}^{(0)}$. In the following, we give a recipe how to construct the coupling factors in (37) for a specific $\chi_{e_{1}} \chi_{e_{2}} \rightarrow X_{A} X_{B} \rightarrow \chi_{e_{4}} \chi_{e_{3}}$ reaction, such that taken together with the kinematic factors in Sec. A.3, they allow to determine the absorptive part of the Wilson coefficients $\hat{f}$ :

1. Draw all tree-level diagrams that contribute to $\chi_{e_{1}} \chi_{e_{2}} \rightarrow X_{A} X_{B}$ and $\chi_{e_{4}} \chi_{e_{3}} \rightarrow$ $X_{A} X_{B}$ annihilation amplitudes, analogous to the generic diagrams sketched in Fig. 9 or Fig. 10, In particular, assign the same fermion flow as indicated for the generic diagrams.

2. Determine the process-specific (axial-) vector and/or (pseudo-) scalar coupling factors, that arise instead of the generic $q_{e i}^{(d)}$ or $r_{e i}^{(d)}$ place-holder expressions at the generic amplitudes' vertex factors. As the $\chi \chi \rightarrow X_{A} X_{B}$ processes may involve Majorana as well as Dirac fermions, and the latter involve a conserved fermion-number flow, note the following rules:

a) If the direction of the fermion-number flow related to a Dirac particle coincides with the direction of the fermion flow (fixed as in Fig. 9 and Fig. 10), the $\chi \chi \rightarrow$ $X_{A} X_{B}$ process specific coupling factors at the vertices are directly deduced 
from the corresponding interaction terms in the underlying Lagrangian. These coupling factors are given later in (44 50).

b) Otherwise, if the fermion-number flow is antiparallel to the indicated fermion flow, vector coupling factors at vertices attached to a Dirac fermion line, are given by a factor -1 times the expression for the vector coupling given in (44) 46). Axial-vector, scalar and pseudo-scalar coupling factors are unchanged with respect to case $a$ ) above.

3. Build all possible two-coupling factor products, including possible signs related to vector couplings, as far as the case in $2 \mathrm{~b}$ ) above applies, that can arise in each single diagram.

4. Multiply each of the two-coupling factor products, that arise in the $\mathcal{A}_{\chi_{e_{1}} \chi_{e_{2}} \rightarrow X_{A} X_{B}}^{(0)}$ amplitude, with the complex conjugate of each of the two-coupling factor products, arising in $\mathcal{A}_{\chi_{e_{4}} \chi_{e_{3}} \rightarrow X_{A} X_{B}}^{(0)}$. As a result, all possible coupling factor combinations that can occur in $\hat{f} \chi_{e_{1}} \chi_{e_{2}} \rightarrow X_{A} X_{B} \rightarrow \chi_{e_{4}} \chi_{e_{3}}$ are obtained.

Rule $2 b$ ) arises in the following way for the case of diagram $s$ in Figs. 9, 10: according to our convention for the fermion flow in Fig. 9, we obtain an expression $-\bar{v}\left(p_{1}\right) \Gamma u\left(p_{2}\right)$ for the incoming particles' spinor chain if the case under $2 b$ ) applies, where $\Gamma$ denotes the involved Dirac-matrix structure. The minus sign accounts for our convention for the order of the external fermion states. This expression can be rewritten as

$$
-\bar{v}\left(p_{1}\right) \Gamma u\left(p_{2}\right)=\bar{v}\left(p_{2}\right) C \Gamma^{T} C^{-1} u\left(p_{1}\right)
$$

wherein $C$ denotes the charge conjugation matrix. Using

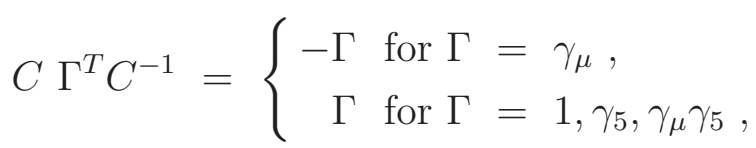

the origin of the minus sign rules for vector couplings under $2 b$ ) above becomes obvious. For diagrams with $t$-channel exchange, a similar derivation also confirms rule $2 b$ ).

Let us introduce the shorthand $a \tilde{a}$ to indicate the diagrams $a$ and $\tilde{a}$ in the $\chi_{e_{1}} \chi_{e_{2}} \rightarrow$ $X_{A} X_{B}$ and $\chi_{e_{4}} \chi_{e_{3}} \rightarrow X_{A} X_{B}$ processes, respectively, to which the coupling factors in a specific coupling factor combination are related. Both $a$ and $\tilde{a}$ can be given by $s, t_{1}$ or $t_{2}$, see Figs. 9 10. Coupling factor combinations originating from $s s$ lead to the $b$ factors, that correspond to the generic selfenergy-amplitude in Fig. 5.6 We label coupling factor combinations, that originate from $t_{1} s, s t_{1}, t_{2} s$ and $s t_{2}$ with the superscript $\alpha=1, \ldots, 4$, respectively. These coupling factor combinations, related to one $t$-channel and one $s$ channel exchange diagram give rise to the $c^{(\alpha)}$ expressions in (37). The $\alpha=1, \ldots, 4$ label-convention for the specific coupling factor combinations allows to correctly allocate

\footnotetext{
${ }^{6}$ Note, that in case of identical particles $X_{A}=X_{B}$, all coupling factor expressions $b$ have to be multiplied with a symmetry factor $1 / 2$, which incorporates the symmetry factor associated with the selfenergy amplitudes in case of identical particles $X_{A}=X_{B}$ in the loop.
} 

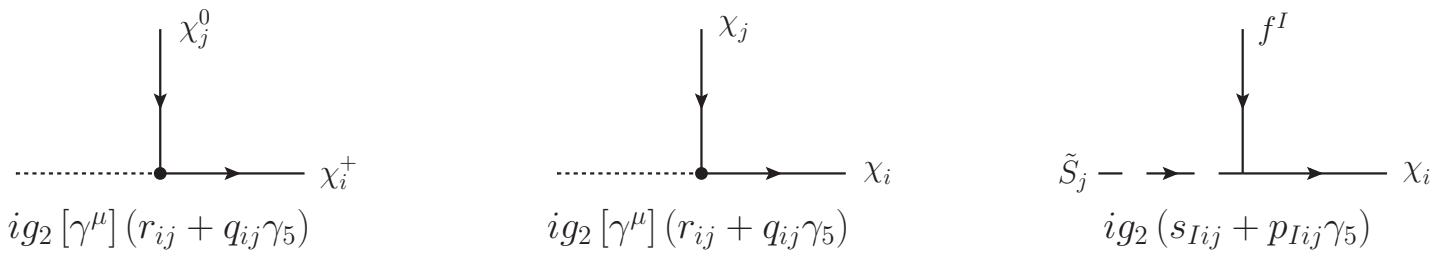

Figure 11: Generic form of the vertex factors in three-point interactions of neutralinos and charginos with SM and Higgs particles, upon which our definition of the (axial-) vector and (pseudo-) scalar coupling factors given in the text is based.

the $c^{(\alpha)}$ to their corresponding generic triangle-amplitude 'triangle $\alpha$ ' in Fig. 66. Coupling factor combinations originating from $t_{1} t_{2}, t_{1} t_{1}, t_{2} t_{1}$ and $t_{2} t_{2}$ are labelled with superscript $\alpha=1, \ldots, 4$, and give rise to the $d^{(\alpha)}$ expressions. As in case of the $c^{(\alpha)}$, this convention correctly assigns $d^{(\alpha)}$ expressions to their corresponding 'box $\alpha$ ' amplitude in Fig. 7 or Fig. 8 .

We introduce the index $n$ in order to label the different coupling factor combinations for a given fixed a $\tilde{a}$. Each individual $n$ is given by a character-string, where the $i$ th character gives the type $(r$ or $q$ ) of the coupling factor which is related to the $i$ th vertex in the particular diagram a a in Figs. 58. The vertices of box-amplitudes are enumerated according to the respective attached external particles $\chi_{e_{i}}, i=1, \ldots, 4$. In case of selfenergy- and triangle-diagrams with inner vertices our vertex-enumeration convention is from top to bottom and left to right. Vertex factors of type $c_{A B X_{i}}$ are not specified in the corresponding string $n$, because the nature of the particles $X_{A}, X_{B}$ and $X_{i}$ involved in the diagram completely characterize that coupling. For triangles with $X_{A} X_{B}=V V, V S$ or $S S$, for example, the index $n$ will range over strings $r r r, q q r, \ldots$, where the characters $r$ or $q$ indicate the type of coupling of the external particles to the $X_{A} X_{B}$ pair and to the single $s$-channel exchanged particle species, see Fig. 6.

To further specify the coupling factor combinations for given $a \tilde{a}$ and $n$, we use the labels $i_{1}$ and $i_{2}$ to indicate the particle species that are internally exchanged in diagrams $a$ and $\tilde{a}$. Therewith, the coupling factor combinations $b_{n i_{1} i_{2}}, c_{n i_{1} i_{2}}^{(\alpha)}$ and $d_{n i_{1} i_{2}}^{(\alpha)}$ that should enter in (37), together with the generic kinematic factor expressions given in App. A.3, can be unambiguously determined.

In order to completely fix our conventions, we summarize in the following the expressions for the (axial-) vector and (pseudo-) scalar coupling factors, that arise in the three-point interactions of charginos and neutralinos with SM and Higgs particles. The definitions of the coupling factors assume that we take $\chi_{i}^{+}$to be the particle and $\chi_{i}^{-}$its anti-particle, such that the Dirac fermion number flow, indicated by the arrow on the Dirac fermion line for a chargino, will always refer to the direction of $\chi_{i}^{+}$flow. The latter convention agrees with that of Rosiek 33.

The generic form for the vertex factor, that describes the 3-point interaction of an incoming neutralino $\chi_{j}^{0}$, an outgoing chargino $\chi_{i}^{+}$and either an incoming charged Higgs 
particle $G^{+}$or $\mathrm{H}^{+}$or an incoming $W^{+}$-boson is given in the left-most diagram in Fig. 11, Note that the gamma matrix $\gamma^{\mu}$ in the vertex factor has to be omitted in case of interactions with the charged Higgs particles. The specific scalar and pseudo-scalar or vector and axial-vector coupling factors, that have to be replaced for the generic $r_{i j}$ and $q_{i j}$ couplings therein read

$$
\begin{array}{r}
s_{i j}^{H_{m}^{+}}\left(p_{i j}^{H_{m}^{+}}\right)=-\frac{1}{2}\left[Z_{H}^{2 m}\left(\widetilde{Z}_{N}^{4 j *} \widetilde{Z}_{+}^{1 i *}+\frac{1}{\sqrt{2}} \widetilde{Z}_{+}^{2 i *}\left(\widetilde{Z}_{N}^{2 j *}+\tan \theta_{W} \widetilde{Z}_{N}^{1 j *}\right)\right)\right. \\
\left. \pm Z_{H}^{1 m}\left(\widetilde{Z}_{N}^{3 j} \widetilde{Z}_{-}^{1 i}-\frac{1}{\sqrt{2}} \widetilde{Z}_{-}^{2 i}\left(\widetilde{Z}_{N}^{2 j}+\tan \theta_{W} \widetilde{Z}_{N}^{1 j}\right)\right)\right] \\
v_{i j}^{W}=\frac{1}{2}\left(\widetilde{Z}_{N}^{2 j *} \widetilde{Z}_{-}^{1 i}+\widetilde{Z}_{N}^{2 j} \widetilde{Z}_{+}^{1 i *}+\frac{1}{\sqrt{2}} \widetilde{Z}_{N}^{3 j *} \widetilde{Z}_{-}^{2 i}-\frac{1}{\sqrt{2}} \widetilde{Z}_{N}^{4 j} \widetilde{Z}_{+}^{2 i *}\right) \\
a_{i j}^{W}=\frac{1}{2}\left(\widetilde{Z}_{N}^{2 j *} \widetilde{Z}_{-}^{1 i}-\widetilde{Z}_{N}^{2 j} \widetilde{Z}_{+}^{1 i *}+\frac{1}{\sqrt{2}} \widetilde{Z}_{N}^{3 j *} \widetilde{Z}_{-}^{2 i}+\frac{1}{\sqrt{2}} \widetilde{Z}_{N}^{4 j} \widetilde{Z}_{+}^{2 i *}\right)
\end{array}
$$

where $H_{1}^{+} \equiv H^{+}$and $H_{2}^{+} \equiv G^{+}$, and the mixing matrices are defined as in Ref. [33. The generic form of the three point interaction of either two neutralinos or two charginos with an electrically neutral gauge boson or Higgs particle is depicted in the second diagram in Fig. 11, Again, the gamma-matrix $\gamma^{\mu}$ has to be omitted in the vertex factor if the corresponding reaction refers to interactions with the neutral Higgs particles. In the case of an incoming $\chi_{j}^{+}$and an outgoing $\chi_{i}^{+}$, the (axial-) vector and (pseudo-) scalar couplings, that encode interactions with the neutral scalar and pseudo-scalar Higgs particles $\left(h^{0}, H^{0}, G^{0}, A^{0}\right)$, the $Z$-boson or the photon are given by the following expressions:

$$
\begin{aligned}
s_{i j}^{H_{m}^{0}}\left(p_{i j}^{H_{m}^{0}}\right) & =-\frac{1}{2 \sqrt{2}}\left[Z_{R}^{1 m}\left(\widetilde{Z}_{-}^{2 j *} \widetilde{Z}_{+}^{1 i *} \pm \widetilde{Z}_{-}^{2 i} \widetilde{Z}_{+}^{1 j}\right)+Z_{R}^{2 m}\left(\widetilde{Z}_{-}^{1 j *} \widetilde{Z}_{+}^{2 i *} \pm \widetilde{Z}_{-}^{1 i} \widetilde{Z}_{+}^{2 j}\right)\right], \\
s_{i j}^{A_{m}^{0}}\left(p_{i j}^{A_{m}^{0}}\right) & =-\frac{i}{2 \sqrt{2}}\left[Z_{H}^{1 m}\left(\widetilde{Z}_{-}^{2 j *} \widetilde{Z}_{+}^{1 i *} \mp \widetilde{Z}_{-}^{2 i} \widetilde{Z}_{+}^{1 j}\right)+Z_{H}^{2 m}\left(\widetilde{Z}_{-}^{1 j *} \widetilde{Z}_{+}^{2 i *} \mp \widetilde{Z}_{-}^{1 i} \widetilde{Z}_{+}^{2 j}\right)\right], \\
v_{i j}^{Z} & =-\frac{1}{4 c_{W}}\left(\widetilde{Z}_{-}^{1 i} \widetilde{Z}_{-}^{1 j *}+\widetilde{Z}_{+}^{1 i *} \widetilde{Z}_{+}^{1 j}+2\left(c_{W}^{2}-s_{W}^{2}\right) \delta_{i j}\right), \\
a_{i j}^{Z} & =\frac{1}{4 c_{W}}\left(\widetilde{Z}_{+}^{1 i *} \widetilde{Z}_{+}^{1 j}-\widetilde{Z}_{-}^{1 i} \widetilde{Z}_{-}^{1 j *}\right), \\
v_{i j}^{\gamma} & =-s_{W} \delta_{i j}, \\
a_{i j}^{\gamma} & =0,
\end{aligned}
$$

where $H_{1}^{0} \equiv H^{0}, H_{2}^{0} \equiv h^{0}$ and $A_{1}^{0} \equiv A^{0}, A_{2}^{0} \equiv G^{0}$. Finally, three-point interactions of an incoming $\chi_{j}^{0}$ and an outgoing $\chi_{i}^{0}$ with a (pseudo-) scalar Higgs particle or the $Z$-boson involve the following (axial-) vector or (pseudo-) scalar coupling factors

$$
s_{i j}^{(0), H_{m}^{0}}\left(p_{i j}^{(0), H_{m}^{0}}\right)=\frac{1}{4}\left[\left(Z_{R}^{2 m} \widetilde{Z}_{N}^{4 i *}-Z_{R}^{1 m} \widetilde{Z}_{N}^{3 i *}\right)\left(\widetilde{Z}_{N}^{2 j *}-\tan \theta_{W} \widetilde{Z}_{N}^{1 j *}\right)+(i \leftrightarrow j)\right] \pm c . c .,
$$




$$
\begin{aligned}
s_{i j}^{(0), A_{m}^{0}}\left(p_{i j}^{(0), A_{m}^{0}}\right) & =\frac{i}{4}\left[\left(Z_{H}^{2 m} \widetilde{Z}_{N}^{4 i *}-Z_{H}^{1 m} \widetilde{Z}_{N}^{3 i *}\right)\left(\widetilde{Z}_{N}^{2 j *}-\tan \theta_{W} \widetilde{Z}_{N}^{1 j *}\right)+(i \leftrightarrow j)\right] \pm c . c . \\
v_{i j}^{(0), Z}\left(a_{i j}^{(0), Z}\right) & =\frac{1}{4 c_{W}}\left(\widetilde{Z}_{N}^{3 i} \widetilde{Z}_{N}^{3 j *}-\widetilde{Z}_{N}^{4 i} \widetilde{Z}_{N}^{4 j *} \mp(i \leftrightarrow j)\right) .
\end{aligned}
$$

The (axial-) vector and (pseudo-) scalar coupling factors in (45) and (46), which are all related to interactions with neutral SM and Higgs particles, satisfy

$$
\begin{array}{ll}
v_{i j}^{*}=v_{j i}, & a_{i j}^{*}=a_{j i}, \\
s_{i j}^{*}=s_{j i}, & p_{i j}^{*}=-p_{j i} .
\end{array}
$$

as a consequence of the hermiticity of the underlying SUSY Lagrangian.

The generic form of the vertex factor for three-point interactions of a neutralino or chargino with a SM fermion and a sfermion is given in the right-most diagram in Fig. 11. In case of interactions of an incoming SM fermion $f^{I}$ with a sfermion $\tilde{S}_{j}$ and an outgoing neutralino $\chi_{i}^{0}$, the specific (pseudo-) scalar couplings, that have to be replaced for the generic $s_{I i j}$ and $p_{I i j}$ expressions in Fig. $11 \mathrm{read}$

$$
\begin{aligned}
s_{I i j}^{u \tilde{U}}\left(p_{I i j}^{u \tilde{U}}\right)= & \frac{1}{\sqrt{2}} q_{u} \tan \theta_{W} \widetilde{Z}_{N}^{1 i *} Z_{U}^{(I+3) j *}-\frac{m_{u}^{I}}{2 \sqrt{2} \sin \beta M_{W}}\left(\widetilde{Z}_{N}^{4 i *} Z_{U}^{I j *} \pm \widetilde{Z}_{N}^{4 i} Z_{U}^{(I+3) j *}\right) \\
& \mp \frac{1}{\sqrt{2}}\left(T_{u} \widetilde{Z}_{N}^{2 i}+\left(q_{u}-T_{u}\right) \widetilde{Z}_{N}^{1 i} \tan \theta_{W}\right) Z_{U}^{I j *}, \\
s_{I i j}^{d \tilde{D}}\left(p_{I i j}^{d \tilde{D}}\right)= & \frac{1}{\sqrt{2}} q_{d} \tan \theta_{W} \widetilde{Z}_{N}^{1 i *} Z_{D}^{(I+3) j}-\frac{m_{d}^{I}}{2 \sqrt{2} \cos \beta M_{W}}\left(\widetilde{Z}_{N}^{3 i *} Z_{D}^{I j} \pm \widetilde{Z}_{N}^{3 i} Z_{D}^{(I+3) j}\right) \\
& \mp \frac{1}{\sqrt{2}}\left(T_{d} \widetilde{Z}_{N}^{2 i}+\left(q_{d}-T_{d}\right) \widetilde{Z}_{N}^{1 i} \tan \theta_{W}\right) Z_{D}^{I j} \\
s_{I i j}^{\nu \tilde{\nu}}\left(p_{I i j}^{\nu \tilde{\nu}}\right)= & \mp \frac{1}{2 \sqrt{2}}\left(\widetilde{Z}_{N}^{2 i}-\widetilde{Z}_{N}^{1 i} \tan \theta_{W}\right) Z_{\nu}^{I j *}, \\
s_{I i j}^{l \tilde{L}}\left(p_{I i j}^{l \tilde{L}}\right)= & \frac{1}{\sqrt{2}} q_{l} \tan \theta_{W} \widetilde{Z}_{N}^{1 i *} Z_{L}^{(I+3) j}-\frac{m_{l}^{I}}{2 \sqrt{2} \cos \beta M_{W}}\left(\widetilde{Z}_{N}^{3 i *} Z_{L}^{I j} \pm \widetilde{Z}_{N}^{3 i} Z_{L}^{(I+3) j}\right) \\
& \mp \frac{1}{\sqrt{2}}\left(T_{l} \widetilde{Z}_{N}^{2 i}+\left(q_{l}-T_{l}\right) \widetilde{Z}_{N}^{1 i} \tan \theta_{W}\right) Z_{L}^{I j} .
\end{aligned}
$$

$I=1,2,3$ denotes the generation index for the fermions, and $j=1, \ldots, 6$ labels the sfermion states $\left(j=1,2,3\right.$ in case of sneutrinos $\left.\tilde{\nu}_{j}\right) . T_{f}$ and $q_{f}$ are defined as

$$
\begin{aligned}
& T_{u}=-T_{d}=-T_{l}=\frac{1}{2} \\
& q_{u}=\frac{2}{3}, \quad q_{d}=-\frac{1}{3}, \quad q_{l}=-1 .
\end{aligned}
$$


The superscripts, $f \tilde{S}$, on the couplings in (48), refer to the fermion $(f)$ - and sfermion $(\tilde{S})$ type involved in the underlying interaction. In case of chargino-fermion-sfermion interactions we find (a sum over repeated indices is implicit)

$$
\begin{aligned}
s_{I i j}^{u \tilde{D}}\left(p_{I i j}^{u \tilde{D}}\right)= & \frac{m_{u}^{I}}{2 \sqrt{2} \sin \beta M_{W}} K^{I J *} \widetilde{Z}_{+}^{2 i *} Z_{D}^{J j} \pm \frac{m_{d}^{J}}{2 \sqrt{2} \cos \beta M_{W}} K^{I J *} \widetilde{Z}_{-}^{2 i} Z_{D}^{(J+3) j} \\
& \mp \frac{1}{2} K^{I J *} \widetilde{Z}_{-}^{1 i} Z_{D}^{J j}, \\
s_{I i j}^{d \tilde{U}}\left(p_{I i j}^{d \tilde{U}}\right)= & \frac{m_{d}^{I}}{2 \sqrt{2} \cos \beta M_{W}} K^{J I} \widetilde{Z}_{-}^{2 i *} Z_{U}^{J j *} \pm \frac{m_{u}^{J}}{2 \sqrt{2} \sin \beta M_{W}} K^{J I} \widetilde{Z}_{+}^{2 i} Z_{U}^{(J+3) j *} \\
& \mp \frac{1}{2} K^{J I} \widetilde{Z}_{+}^{1 i} Z_{U}^{J j *}, \\
s_{I i j}^{\nu \tilde{L}}\left(p_{I i j}^{\nu \tilde{L}}\right)= & \pm \frac{m_{l}^{I}}{2 \sqrt{2} \cos \beta M_{W}} \widetilde{Z}_{-}^{2 i} Z_{L}^{(I+3) j} \mp \frac{1}{2} \widetilde{Z}_{-}^{1 i} Z_{L}^{I j}, \\
s_{I i j}^{l \tilde{\nu}}\left(p_{I i j}^{l \tilde{\nu}}\right)= & \frac{m_{l}^{I}}{2 \sqrt{2} \cos \beta M_{W}} \widetilde{Z}_{-}^{2 i *} Z_{\nu}^{J j *} \mp \frac{1}{2} \widetilde{Z}_{+}^{1 i} Z_{\nu}^{I j *} .
\end{aligned}
$$

The coupling factors with $f \tilde{S}=u \tilde{D}, \nu \tilde{L}$ refer to the interaction of an incoming up-type quark $\left(u^{I}\right)$ or neutralino $\left(\nu^{I}\right)$ with a $\tilde{D}_{j^{-}}$or $\tilde{L}_{j}$-sfermion and an outgoing $\chi_{i}^{+}$. In case of $f \tilde{S}=d \tilde{U}$, l $\tilde{\nu}$, the coupling factors in (50) are related to interactions of an incoming

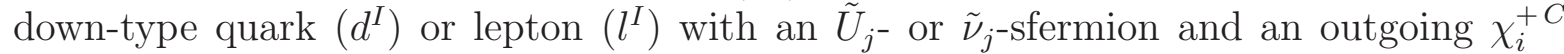
(denoting the charge-conjugate field of $\chi_{i}^{+}$, see [33]).

For the specific $c_{A B X_{i}}$ factors that emerge at the three-point vertex of the $X_{A} X_{B}$ particle pair with the single $s$-channel exchanged particle $X_{i}$ in Fig. 9, we refer to the Feynman rules in [33]: a specific $c_{A B X_{i}}$ is obtained as the factor that multiplies the structure $i g_{2} L_{A B X_{i}}$ in the respective Feynman rule therein. The generic forms of the Lorentz structures $L_{A B X_{i}}$ are collected in Tab. 4. Finally, (axial-) vector and (pseudo-) scalar coupling factors $r$ and $q$ of two SM fermions with a gauge- or Higgs-boson (see Fig. 10) can be directly taken from the corresponding Feynman rules in 33.

In order to illustrate how the above rules should be applied, let us consider an example. Suppose we wish to know the coupling factors $c_{n, i_{1} V}^{(\alpha)}, \alpha=1, \ldots 4$, of diagrams with $s$-channel exchange of a $Z$-boson for the $\chi_{e_{1}}^{-} \chi_{e_{2}}^{+} \rightarrow W^{+} G^{-} \rightarrow \chi_{e_{4}}^{0} \chi_{e_{3}}^{0}$ processes. Following the recipe above, we draw all tree-level diagrams for the $\chi_{e_{1}}^{-} \chi_{e_{2}}^{+} \rightarrow W^{+} G^{-}$as well as the $\chi_{e_{4}}^{0} \chi_{e_{3}}^{0} \rightarrow W^{+} G^{-}$process and assign the same fermion flow as given in the corresponding generic diagrams, Fig. 9, Referring to that fixed fermion flow, we determine the following vertex factors in the diagrams $t_{1}$ and $s$, associated with tree-level $\chi_{e_{1}}^{-} \chi_{e_{2}}^{+} \rightarrow W^{+} G^{-}$ annihilations:

$$
\begin{aligned}
V_{e_{1} i_{1}}^{\beta\left(t_{1}\right)} & =s_{e_{1} i_{1}}^{G}+p_{e_{1} i_{1}}^{G} \gamma_{5}, & V_{e_{2} i_{1}}^{\alpha\left(t_{1}\right)} & =\gamma^{\alpha}\left(-v_{e_{2} i_{1}}^{W *}+a_{e_{2} i_{1}}^{W *} \gamma_{5}\right), \\
V_{e_{1} e_{2}}^{\mu(s)} & =\gamma^{\mu}\left(-v_{e_{1} e_{2}}^{Z}+a_{e_{1} e_{2}}^{Z} \gamma_{5}\right), & c_{W G Z} & =-\frac{s_{W}^{2}}{c_{W}^{2}} .
\end{aligned}
$$




\begin{tabular}{c|c}
\hline$X_{A} X_{B} X_{i}$ & $L_{A B X_{i}}$ \\
\hline \hline$V_{\alpha} V_{\beta} V_{\mu}$ & $g^{\alpha \beta}\left(k_{A}-k_{B}\right)^{\mu}+g^{\mu \alpha}\left(k_{i}-k_{A}\right)^{\beta}+g^{\beta \mu}\left(k_{B}-k_{i}\right)^{\alpha}$ \\
$V_{\alpha} V_{\beta} S$ & $M_{W} g^{\alpha \beta}$ \\
$V_{\alpha} S S$ & $\left(k_{B}-k_{i}\right)^{\alpha}$ \\
$S S V_{\mu}$ & $\left(k_{B}-k_{A}\right)^{\mu}$ \\
$S S S$ & $M_{W}$ \\
$\eta \bar{\eta} V_{\mu}$ & $k_{B}^{\mu}$ \\
$\eta \bar{\eta} S$ & $M_{W}$ \\
\hline
\end{tabular}

Table 4: The generic form of the Lorentz structures $L_{A B X_{i}}$, that are part of the Feynman rule $i g_{2} c_{A B X_{i}} L_{A B X_{i}}$ for the $X_{A} X_{B} X_{i}$ three-point vertex in Fig 9. We assume all fourmomenta, $k_{A}, k_{B}, k_{i}$, to be outgoing at the vertex. The case of $X_{A} X_{B} X_{i}=V_{\alpha} S V_{\mu}$ is trivially related to the case $V_{\alpha} V_{\beta} S$.

The coupling factors are those from (44) and (45)). Note that there is no $t$-channel exchange diagram $t_{2}$ for the above process, as it is forbidden by charge conservation. Further, note that the sign in front of the vector-coupling factor in $V_{e_{2} i_{1}}^{\alpha\left(t_{1}\right)}$ and $V_{e_{1} e_{2}}^{\mu(s)}$ follows from rule $2 b$ ) above. In case of diagrams contributing to $\chi_{e_{4}}^{0} \chi_{e_{3}}^{0} \rightarrow W^{+} G^{-}$we find

$$
\begin{aligned}
V_{e_{4} i_{1}}^{\beta\left(t_{1}\right)} & =s_{i_{1} e_{4}}^{G}+p_{i_{1} e_{4}}^{G} \gamma_{5}, & V_{e_{3} i_{1}}^{\alpha\left(t_{1}\right)} & =\gamma^{\alpha}\left(v_{i_{1} e_{3}}^{W *}+a_{i_{1} e_{3}}^{W *} \gamma_{5}\right), \\
V_{e_{4} i_{1}}^{\alpha\left(t_{2}\right)} & =\gamma^{\alpha}\left(-v_{i_{1} e_{4}}^{W *}+a_{i_{1} e_{4}}^{W *} \gamma_{5}\right), & V_{e_{3} i_{1}}^{\beta\left(t_{2}\right)} & =s_{i_{1} e_{3}}^{G}+p_{i_{1} e_{3}}^{G} \gamma_{5}, \\
V_{e_{4} e_{3}}^{\mu(s)} & =\gamma^{\mu}\left(v_{e_{3} e_{4}}^{(0) Z}+a_{e_{3} e_{4}}^{(0) Z} \gamma_{5}\right), & c_{W G Z} & =-\frac{s_{W}^{2}}{c_{W}^{2}} .
\end{aligned}
$$

To obtain the building blocks for the non-vanishing $c_{n, i_{1} V}^{(\alpha)}$ with $\alpha=1$, one has to combine the coupling factor expressions in the first row of (51) (the factors related to diagram $t_{1}$ in $\chi_{e_{1}}^{-} \chi_{e_{2}}^{+} \rightarrow W^{+} G^{-}$annihilations) with the coupling factor expressions in the last row of (52) (expressions originating from diagram $s$ in $\chi_{e_{4}}^{0} \chi_{e_{3}}^{0} \rightarrow W^{+} G^{-}$), as $\alpha=1$ refers to the $t_{1} s$ product of tree-level diagrams. Similarly, for $\alpha=2$ and 4 , one has to build the combinations of expressions referring to $s t_{1}$ and $s t_{2}$. Therefore, the building-blocks for the non-vanishing $c_{n i_{1} V}^{(\alpha)}$ related to single $s$-channel $V=Z$ exchange read:

$$
\begin{aligned}
& \alpha=1: \quad\left\{\left\{s_{e_{1} i_{1}}^{G}, p_{e_{1} i_{1}}^{G}\right\},\left\{-v_{e_{2} i_{1}}^{W *}, a_{e_{2} i_{1}}^{W *}\right\},\left\{v_{e_{3} e_{4}}^{(0) Z *}, a_{e_{3} e_{4}}^{(0) Z *}\right\},\left\{-\frac{s_{W}^{2}}{c_{W}^{2}}\right\}\right\}, \\
& \alpha=2: \quad\left\{\left\{-v_{e_{1} e_{2}}^{Z}, a_{e_{1} e_{2}}^{Z}\right\},\left\{v_{i_{1} e_{3}}^{W}, a_{i_{1} e_{3}}^{W}\right\},\left\{s_{i_{1} e_{4}}^{G *}, p_{i_{1} e_{4}}^{G *}\right\},\left\{-\frac{s_{W}^{2}}{c_{W}^{2}}\right\}\right\}, \\
& \alpha=4: \quad\left\{\left\{-v_{e_{1} e_{2}}^{Z}, a_{e_{1} e_{2}}^{Z}\right\},\left\{s_{i_{1} e_{3}}^{G *}, p_{i_{1} e_{3}}^{G *}\right\},\left\{-v_{i_{1} e_{4}}^{W}, a_{i_{1} e_{4}}^{W}\right\},\left\{-\frac{s_{W}^{2}}{c_{W}^{2}}\right\}\right\} .
\end{aligned}
$$


In selecting one element from each of the above given subsets and multiplying the selected elements for fixed $\alpha$ with each other, the $c_{n, i_{1} V}^{(\alpha)}$ expressions in $\chi_{e_{1}}^{-} \chi_{e_{2}}^{+} \rightarrow W^{+} G^{-} \rightarrow \chi_{e_{4}}^{0} \chi_{e_{3}}^{0}$ reactions are obtained. Proceeding in that way, we obtain eight different coupling factor combinations for fixed $\alpha$, that are labelled with index $n$. Following our convention for this label, $n$ ranges over $n=r r r, r r q, r q r, q r r, r q q, q r q, q q r, q q q$. The $c_{q q r, i_{1} V}^{(2)}$ expression, for example, reads

$$
c_{q q r, i_{1} V}^{(2)}=-\frac{s_{W}^{2}}{c_{W}^{2}} a_{e_{1} e_{2}}^{Z} a_{i_{1} e_{3}}^{W} s_{i_{1} e_{4}}^{G *} .
$$

\section{A.3 Kinematic factors}

The kinematic factor expressions that refer to a specific $\chi_{e_{1}} \chi_{e_{2}} \rightarrow X_{A} X_{B} \rightarrow \chi_{e_{4}} \chi_{e_{3}}$ scattering reaction depend on the external particles' mass scales $m, \bar{m}$ and $M=m+\bar{m}$. We remind the reader of our convention (see Sec. 2.4)

$$
\begin{aligned}
m & =\frac{m_{e_{1}}+m_{e_{4}}}{2}, & \bar{m} & =\frac{m_{e_{2}}+m_{e_{3}}}{2}, \\
\delta m & =\frac{m_{e_{4}}-m_{e_{1}}}{2}, & \delta \bar{m} & =\frac{m_{e_{3}}-m_{e_{2}}}{2} .
\end{aligned}
$$

Further recall that we expand the scattering amplitudes in $\delta m, \delta \bar{m}$ and count these quantities as $\mathcal{O}\left(v^{2}\right)$. Hence, for the leading-order $S$-wave results presented below, the mass differences $\delta m=\delta \bar{m}=0$, such that there are only two mass scales, $m$ and $\bar{m}$, left, which characterize the external chargino or neutralino states. The masses of the particles $X_{A}$ and $X_{B}$ will be denoted with $m_{A}$ and $m_{B}$. Let us introduce the general notation $\widehat{m}_{a}$ for the rescaling of any mass $m_{a}$ in units of the mass scale $M$,

$$
\widehat{m}_{a}=\frac{m_{a}}{M}
$$

Define the dimensionless quantities

$$
\begin{aligned}
\Delta_{A B} & =\widehat{m}_{A}^{2}-\widehat{m}_{B}^{2}, \\
\beta & =\sqrt{1-2\left(\widehat{m}_{A}^{2}+\widehat{m}_{B}^{2}\right)+\Delta_{A B}^{2}},
\end{aligned}
$$

where in case that $X_{A}=X_{B}, \beta$ is the leading-order term in the expansion of the velocity of the $X_{A} X_{B}$ particle pair in the non-relativistic momenta and mass differences. The expansion of single $s$-channel (gauge or Higgs boson $X_{i}$ ) exchange propagators in $\delta m$, $\delta \bar{m}$ and the non-relativistic 3 -momenta leads to the following denominator-structure at leading order:

$$
P_{i}^{s}=1-\widehat{m}_{i}^{2} .
$$


Similarly, the leading-order expansion of $t$ - and $u$-channel gaugino and sfermion propagators in $\delta m, \delta \bar{m}$ and the non-relativistic 3-momenta gives rise to the denominatorstructures

$$
\begin{aligned}
P_{i A B} & =\widehat{m} \hat{m}+\widehat{m}_{i}^{2}-\widehat{m} \widehat{m}_{A}^{2}-\widehat{\bar{m}} \widehat{m}_{B}^{2}, \\
P_{i B A} & =\left.P_{i A B}\right|_{A \leftrightarrow B} .
\end{aligned}
$$

Using the above definitions, the kinematic factors for the leading order $S$-wave Wilson coefficients related to the selfenergy-topology in Fig. 5 are conveniently written as

$$
B_{n, i_{1} i_{2}}^{X_{A} X_{B}}\left({ }^{2 s+1} S_{J}\right)=\frac{\beta}{P_{i_{1}}^{s} P_{i_{2}}^{s}} \tilde{B}_{n, i_{1} i_{2}}^{X_{A} X_{B}}\left({ }^{2 s+1} S_{J}\right),
$$

where the labels $i_{1}$ and $i_{2}$ refer to the particle species that are exchanged in the left and right $s$-channel propagator. As generically either gauge-boson $(V)$ or Higgs $(S) s$-channel exchange occurs in the processes under consideration, the combination $i_{1} i_{2}$ is given by $i_{1} i_{2}=V V, V S, S V, S S$. Kinematic factors arising from the triangle-topologies shown in Fig. [6] have the following generic form

$$
\begin{aligned}
& C_{n, i_{1} X}^{(\alpha) X_{A} X_{B}}\left({ }^{2 s+1} S_{J}\right)=\frac{\beta}{P_{i_{1} A B} P_{X}^{s}} \tilde{C}_{n, i_{1} X}^{(\alpha) X_{A} X_{B}}\left({ }^{2 s+1} S_{J}\right) \quad \alpha=1,2, \\
& C_{n, i_{1} X}^{(\alpha) X_{A} X_{B}}\left({ }^{2 s+1} S_{J}\right)=\frac{\beta}{P_{i_{1} B A} P_{X}^{s}} \tilde{C}_{n, i_{1} X}^{(\alpha) X_{A} X_{B}}\left({ }^{2 s+1} S_{J}\right) \quad \alpha=3,4 .
\end{aligned}
$$

The index $i_{1}$ in the above expressions is related to the $t$ - or $u$-channel exchanged particle species, whereas the subscript-index $X$ indicates the type of the single $s$-channel exchanged particle-species, $X=V, S$. Finally, kinematic factors associated with the box-topologies of Fig. 7 and Fig. 8 generically read

$$
\begin{aligned}
& D_{n, i_{1} i_{2}}^{(1) X_{A} X_{B}}\left({ }^{2 s+1} S_{J}\right)=\frac{\beta}{P_{i_{1} A B} P_{i_{2} B A}} \tilde{D}_{n, i_{1} i_{2}}^{(1) X_{A} X_{B}}\left({ }^{2 s+1} S_{J}\right) \\
& D_{n, i_{1} i_{2}}^{(2) X_{A} X_{B}}\left({ }^{2 s+1} S_{J}\right)=\frac{\beta}{P_{i_{1} A B} P_{i_{2} A B}} \tilde{D}_{n, i_{1} i_{2}}^{(2) X_{A} X_{B}}\left({ }^{2 s+1} S_{J}\right) \\
& D_{n, i_{1} i_{2}}^{(3) X_{A} X_{B}}\left({ }^{2 s+1} S_{J}\right)=\frac{\beta}{P_{i_{1} B A} P_{i_{2} A B}} \tilde{D}_{n, i_{1} i_{2}}^{(3) X_{A} X_{B}}\left({ }^{2 s+1} S_{J}\right) \\
& D_{n, i_{1} i_{2}}^{(4) X_{A} X_{B}}\left({ }^{2 s+1} S_{J}\right)=\frac{\beta}{P_{i_{1} B A} P_{i_{2} B A}} \tilde{D}_{n, i_{1} i_{2}}^{(4) X_{A} X_{B}}\left({ }^{2 s+1} S_{J}\right)
\end{aligned}
$$

Indices $i_{1}$ and $i_{2}$ in (64) refer to the exchanged particle species in the left and right $t$ and $u$-channels of the 1-loop box-amplitudes, respectively.

Throughout this appendix, the labels $A$ and $B$ are related to the particles $X_{A}$ and $X_{B}$. Recall that these are the actual final-state particles in a $\chi_{i} \chi_{j} \rightarrow X_{A} X_{B}$ (treelevel) annihilation reaction. The overall prefactors in (62 64) arise from the phasespace integration $(\beta)$ and from the leading-order expansion of $s$ - or $t$ - and $u$-channel propagators in the non-relativistic limit. 
Finally recall, that each individual index $n$ in (62 64) is given by a character string, whose elements indicate the type $(r$ or $q$ ) of the corresponding generic coupling structures at the vertices of the respective underlying 1-loop amplitude in Figs. 56 8, In the results that we quote next we only write explicitly the kinematic factors for those $n$ which are non-vanishing.

\section{A.3.1 Kinematic factors for $X_{A} X_{B}=V V$}

The kinematic factors $\tilde{B}_{n, i_{1} i_{2}}^{V V}$ in case of ${ }^{1} S_{0}$ partial wave reactions are given by

$$
\begin{aligned}
\tilde{B}_{q q, V V}^{V V}\left({ }^{1} S_{0}\right) & =-\frac{\beta^{2}}{2}+3 \Delta_{A B}^{2}, \\
\tilde{B}_{q q, V S}^{V V}\left({ }^{1} S_{0}\right) & =\tilde{B}_{q q, S V}^{V V}\left({ }^{1} S_{0}\right)=3 \widehat{m}_{W} \Delta_{A B}, \\
\tilde{B}_{q q, S S}^{V V}\left({ }^{1} S_{0}\right) & =4 \widehat{m}_{W}^{2} .
\end{aligned}
$$

In case of ${ }^{3} S_{1}$ partial-wave reactions we have

$$
\tilde{B}_{r r, V V}^{V V}\left({ }^{3} S_{1}\right)=-\frac{9}{2}+\frac{4}{3} \beta^{2}-\frac{1}{2} \Delta_{A B}^{2}
$$

Only the kinematic factors $\tilde{B}_{n, i_{1} i_{2}}^{V V}$ given explicitly in (65) with $n=r r, q q$ are nonvanishing. In case of $X_{A} X_{B}=V V$, the kinematic factors for the triangle- and boxdiagram topologies $\alpha=3,4$ are related to the corresponding expressions for diagramtopologies $\alpha=1,2$ (see Figs. 6 17). The relations read

$$
\begin{aligned}
& \tilde{C}_{n, i_{1} V}^{(3) V V}\left({ }^{2 s+1} S_{J}\right)=-\left.\tilde{C}_{n, i_{1} V}^{(1) V V}\left({ }^{2 s+1} S_{J}\right)\right|_{A \leftrightarrow B}, \\
& \tilde{C}_{n, i_{1} V}^{(4) V V}\left({ }^{2 s+1} S_{J}\right)=-\left.\tilde{C}_{n, i_{1} V}^{(2) V V}\left({ }^{2 s+1} S_{J}\right)\right|_{A \leftrightarrow B}, \\
& \tilde{C}_{n, i_{1} S}^{(3) V V}\left({ }^{2 s+1} S_{J}\right)=\left.\tilde{C}_{n, i_{1} S}^{(1) V V}\left({ }^{2 s+1} S_{J}\right)\right|_{A \leftrightarrow B}, \\
& \tilde{C}_{n, i_{1} S}^{(4) V V}\left({ }^{2 s+1} S_{J}\right)=\left.\tilde{C}_{n, i_{1} S}^{(2) V V}\left({ }^{2 s+1} S_{J}\right)\right|_{A \leftrightarrow B}, \\
& \tilde{D}_{n, i_{1} i_{2}}^{(3) V V}\left({ }^{2 s+1} S_{J}\right)=\left.\tilde{D}_{n, i_{1} i_{2}}^{(1) V V}\left({ }^{2 s+1} S_{J}\right)\right|_{A \leftrightarrow B}, \\
& \tilde{D}_{n, i_{1} i_{2}}^{(4) V V}\left({ }^{2 s+1} S_{J}\right)=\left.\tilde{D}_{n, i_{1} i_{2}}^{(2) V V}\left({ }^{2 s+1} S_{J}\right)\right|_{A \leftrightarrow B} .
\end{aligned}
$$

The minus sign in the relation for the triangle coefficients $\tilde{C}_{n, i_{1} V}^{(\alpha) V V}$ in (69) arises from interchanging the two gauge-bosons $X_{A}$ and $X_{B}$ at the internal three-gauge boson vertex. The expressions $\tilde{C}_{n, i_{1} V}^{(\alpha) V V}$ for diagram-topologies $\alpha=1,2$, that refer to leading-order ${ }^{1} S_{0}$ partial waves read

$$
\begin{aligned}
& \tilde{C}_{r q q, i_{1} V}^{(1) V V}\left({ }^{1} S_{0}\right)=\frac{\beta^{2}}{2}-\frac{3}{2}(\widehat{m}-\widehat{\bar{m}}) \Delta_{A B}-\frac{3}{2} \Delta_{A B}^{2}+3 \widehat{m}_{i_{1}} \Delta_{A B}, \\
& \tilde{C}_{q q r, i_{1} V}^{(2) V V}\left({ }^{1} S_{0}\right)=\tilde{C}_{r q q, i_{1} V}^{(1) V V}\left({ }^{1} S_{0}\right) .
\end{aligned}
$$


In case of $C_{n, i_{1} V}^{(\alpha) V V}$ expressions related to ${ }^{3} S_{1}$ partial waves and diagram-topologies $\alpha=1,2$ we find

$$
\tilde{C}_{r r r, i_{1} V}^{(\alpha) V V}\left({ }^{3} S_{1}\right)=-\frac{5}{6} \beta^{2}+(\widehat{m}-\widehat{m}) \frac{\Delta_{A B}}{2}+\frac{\Delta_{A B}^{2}}{2}+3 \widehat{m}_{i_{1}}
$$

We deduce the following expressions for $\tilde{C}_{n, i_{1} S}^{(\alpha) V V}$ coefficients and diagram topologies $\alpha=$ 1, 2:

$$
\tilde{C}_{r q q, i_{1} S}^{(1) V V}\left({ }^{1} S_{0}\right)=\tilde{C}_{q q r, i_{1} S}^{(2) V V}\left({ }^{1} S_{0}\right)=-\widehat{m}_{W}\left(\widehat{m}-\widehat{\bar{m}}+\Delta_{A B}\right)+4 \widehat{m}_{W} \widehat{m}_{i_{1}}
$$

There are additional non-vanishing $C_{n, i_{1} X}^{(\alpha) V V}$ expressions for $X=V, S$ in both the case of ${ }^{1} S_{0}$ and ${ }^{3} S_{1}$ partial wave reactions, which are related to the expressions in $(70,73)$ in the following way:

$$
\begin{aligned}
& \tilde{C}_{q q r, i_{1} X}^{(1) V V}\left({ }^{2 s+1} S_{J}\right)=\tilde{C}_{r q q, i_{1} X}^{(2) V V}\left({ }^{2 s+1} S_{J}\right)=\left.\tilde{C}_{r r r, i_{1} X}^{(1) V V}\left({ }^{2 s+1} S_{J}\right)\right|_{m_{i_{1}} \rightarrow-m_{i_{1}}}, \\
& \tilde{C}_{q r q, i_{1} X}^{(1) V V}\left({ }^{2 s+1} S_{J}\right)=\tilde{C}_{q r q, i_{1} X}^{(2) V V}\left({ }^{2 s+1} S_{J}\right)=\left.\tilde{C}_{r q q, i_{1} X}^{(1) V V}\left({ }^{2 s+1} S_{J}\right)\right|_{m_{i_{1}} \rightarrow-m_{i_{1}}} .
\end{aligned}
$$

Turning to terms related to box-diagrams, the non-vanishing expressions $\tilde{D}_{n, i_{1} i_{2}}^{(\alpha) V V}$ for $\alpha=1,2$ are given by

$$
\begin{aligned}
\tilde{D}_{r r r r, i_{1} i_{2}}^{(\alpha) V V}\left({ }^{1} S_{0}\right)= & \frac{\beta^{2}}{2} \\
\tilde{D}_{r q q r, i_{1} i_{2}}^{(1) V V}\left({ }^{1} S_{0}\right)= & \frac{\beta^{2}}{2}+(\widehat{m}-\widehat{\bar{m}})^{2}-\Delta_{A B}^{2}+4 \widehat{m}_{i_{1}} \widehat{m}_{i_{2}} \\
& -\widehat{m}_{i_{1}}\left(\widehat{m}-\widehat{\bar{m}}-\Delta_{A B}\right)-\widehat{m}_{i_{2}}\left(\widehat{m}-\widehat{\bar{m}}+\Delta_{A B}\right), \\
\tilde{D}_{r q q r, i_{1} i_{2}}^{(2), V V}\left({ }^{1} S_{0}\right)= & -\frac{\beta^{2}}{2}+\left(\widehat{m}-\widehat{\bar{m}}+\Delta_{A B}\right)^{2}+4 \widehat{m}_{i_{1}} \widehat{m}_{i_{2}} \\
& -\left(\widehat{m}_{i_{1}}+\widehat{m}_{i_{2}}\right)\left(\widehat{m}-\widehat{\bar{m}}+\Delta_{A B}\right),
\end{aligned}
$$

and

$$
\begin{aligned}
\tilde{D}_{r r r r, i_{1} i_{2}}^{(1) V V}\left({ }^{3} S_{1}\right)= & -\frac{2}{3} \beta^{2}-\frac{1}{2}(\widehat{m}-\widehat{\bar{m}})^{2}+\frac{1}{2} \Delta_{A B}^{2}+2 \widehat{m}_{i_{1}} \widehat{m}_{i_{2}}, \\
\tilde{D}_{r r r r, i_{1} i_{2}}^{(2) V V}\left({ }^{3} S_{1}\right)= & \frac{2}{3} \beta^{2}-\frac{1}{2}\left(\widehat{m}-\widehat{\bar{m}}+\Delta_{A B}\right)^{2}-2 \widehat{m}_{i_{1}} \widehat{m}_{i_{2}}, \\
\tilde{D}_{r q q r, i_{1} i_{2}}^{(1) V V}\left({ }^{3} S_{1}\right)= & -\frac{1}{3} \beta^{2}-\frac{1}{2}(\widehat{m}-\widehat{m})^{2}+\frac{1}{2} \Delta_{A B}^{2}-2 \widehat{m}_{i_{1}} \widehat{m}_{i_{2}} \\
& +\widehat{m}_{i_{1}}\left(\widehat{m}-\widehat{m}-\Delta_{A B}\right)+\widehat{m}_{i_{2}}\left(\widehat{m}-\widehat{m}+\Delta_{A B}\right), \\
\tilde{D}_{r q q r, i_{1} i_{2}}^{(2) V V}\left({ }^{3} S_{1}\right)= & -\frac{1}{3} \beta^{2}+\frac{1}{2}\left(m-\bar{m}+\widehat{\Delta}_{A B}\right)^{2}+2 \widehat{m}_{i_{1}} \widehat{m}_{i_{2}}
\end{aligned}
$$




$$
-\widehat{m}_{i_{1}}\left(\widehat{m}-\widehat{\bar{m}}+\Delta_{A B}\right)-\widehat{m}_{i_{2}}\left(\widehat{m}-\widehat{\bar{m}}+\Delta_{A B}\right)
$$

The remaining non-vanishing kinematic factors $\tilde{D}_{n, i_{1} i_{2}}^{V V}$ for both spin-0 and spin-1 $\chi \chi$ states are related to the expressions given above by

$$
\begin{aligned}
& \tilde{D}_{q q q q, i_{1} i_{2}}^{(\alpha) V V}\left({ }^{2 s+1} S_{J}\right)=\tilde{D}_{r r r r, i_{1} i_{2}}^{(\alpha) V V}\left({ }^{2 s+1} S_{J}\right), \\
& \tilde{D}_{r r q q, i_{1} i_{2}}^{(\alpha) V V}\left({ }^{2 s+1} S_{J}\right)=\tilde{D}_{q q r r, i_{1} i_{2}}^{(\alpha) V V}\left({ }^{2 s+1} S_{J}\right)=\left.\tilde{D}_{r r r r, i_{1} i_{2}}^{(\alpha) V V}\left({ }^{2 s+1} S_{J}\right)\right|_{\hat{m}_{i_{1}} \hat{m}_{i_{2}} \rightarrow-\hat{m}_{i_{1}} \hat{m}_{i_{2}}}, \\
& \tilde{D}_{q r r q, i_{1} i_{2}}^{(\alpha) V V}\left({ }^{2 s+1} S_{J}\right)=\left.\tilde{D}_{r q q r, i_{1} i_{2}}^{(\alpha) V V}\left({ }^{2 s+1} S_{J}\right)\right|_{\hat{m}_{i_{1,2}} \rightarrow-\hat{m}_{i_{1}, 2}}, \\
& \tilde{D}_{r q r q, i_{1} i_{2}}^{(\alpha) V V}\left({ }^{2 s+1} S_{J}\right)=\left.\tilde{D}_{r q q r, i_{1} i_{2}}^{(\alpha) V V}\left({ }^{2 s+1} S_{J}\right)\right|_{\hat{m}_{i_{2}} \rightarrow-\hat{m}_{i_{2}}}, \\
& \tilde{D}_{q r q r, i_{1} i_{2}}^{(\alpha) V V}\left({ }^{2 s+1} S_{J}\right)=\left.\tilde{D}_{r q q r, i_{1} i_{2}}^{(\alpha) V V}\left({ }^{2 s+1} S_{J}\right)\right|_{\hat{m}_{i_{1}} \rightarrow-\hat{m}_{i_{1}}} .
\end{aligned}
$$

The notation in the second line of (82) means that the product $\widehat{m}_{i_{1}} \widehat{m}_{i_{2}}$ is replaced, but all other appearances of either $\widehat{m}_{i_{1}}$ or $\widehat{m}_{i_{2}}$ are untouched.

\section{A.3.2 Kinematic factors for $X_{A} X_{B}=V S$}

We find the following expressions for $\tilde{B}_{n, i_{1} i_{2}}^{V S}$ terms in ${ }^{1} S_{0}$ partial-wave reactions with $i_{1} i_{2}=V V, V S, S V, S S$ :

$$
\begin{aligned}
& \tilde{B}_{q q, V V}^{V S}\left({ }^{1} S_{0}\right)=-\widehat{m}_{W}^{2} \\
& \tilde{B}_{q q, V S}^{V S}\left({ }^{1} S_{0}\right)=\tilde{B}_{q q, S V}^{V S}\left({ }^{1} S_{0}\right)=\frac{\widehat{m}_{W}}{2}\left(-3+\Delta_{A B}\right), \\
& \tilde{B}_{q q, S S}^{V S}\left({ }^{1} S_{0}\right)=\frac{\beta^{2}}{4}-\frac{9}{4}+\frac{3}{2} \Delta_{A B}-\frac{\Delta_{A B}^{2}}{4} .
\end{aligned}
$$

In case of ${ }^{3} S_{1}$ partial-wave processes the corresponding $\tilde{B}_{n, i_{1} i_{2}}^{V S}$ coefficients read

$$
\tilde{B}_{r r, V V}^{V S}\left({ }^{3} S_{1}\right)=\widehat{m}_{W}^{2} \text {. }
$$

Kinematic factors $\tilde{C}_{n, i_{1} V}^{(\alpha) V S}$, that are related to the four generic triangle-topologies $\alpha$ with gauge-boson $(V)$ exchange in the single $s$-channel (see Fig. 6) are given by

$$
\begin{aligned}
& \tilde{C}_{r q q, i_{1} V}^{(1) V S}\left({ }^{1} S_{0}\right)=\tilde{C}_{q q r, i_{1} V}^{(2) V S}\left({ }^{1} S_{0}\right)=-\frac{\widehat{m}_{W}}{2}\left(\widehat{m}-\widehat{\bar{m}}+\Delta_{A B}\right)-\widehat{m}_{W} \widehat{m}_{i_{1}}, \\
& \tilde{C}_{r q q, i_{1} V}^{(3) V S}\left({ }^{1} S_{0}\right)=\tilde{C}_{q q r, i_{1} V}^{(4) V S}\left({ }^{1} S_{0}\right)=\frac{\widehat{m}_{W}}{2}\left(\widehat{m}-\widehat{\bar{m}}-\Delta_{A B}\right)+\widehat{m}_{W} \widehat{m}_{i_{1}},
\end{aligned}
$$

as well as

$$
\begin{aligned}
& \tilde{C}_{r r r, i_{1} V}^{(1) V S}\left({ }^{3} S_{1}\right)=\tilde{C}_{r r r, i_{1} V}^{(2) V S}\left({ }^{3} S_{1}\right)=-\tilde{C}_{r q q, i_{1} V}^{(1) V S}\left({ }^{1} S_{0}\right), \\
& \tilde{C}_{r r r, i_{1} V}^{(3) V S}\left({ }^{3} S_{1}\right)=\tilde{C}_{r r r, i_{1} V}^{(4) V S}\left({ }^{3} S_{1}\right)=-\left.\tilde{C}_{r q q, i_{1} V}^{(3) V S}\left({ }^{1} S_{0}\right)\right|_{\widehat{m}_{i_{1}} \rightarrow-\widehat{m}_{i_{1}}} .
\end{aligned}
$$


In case of $\tilde{C}_{n, i_{1} S}^{(\alpha) V S}$ expressions we find

$$
\begin{aligned}
\tilde{C}_{r q q, i_{1} S}^{(1) V S}\left({ }^{1} S_{0}\right)= & -\frac{\beta^{2}}{4}-\frac{3}{4}(\widehat{m}-\widehat{\bar{m}})+(\widehat{m}-\widehat{\bar{m}}-3) \frac{\Delta_{A B}}{4} \\
& +\frac{\Delta_{A B}^{2}}{4}-\frac{\widehat{m}_{i_{1}}}{2}\left(3-\Delta_{A B}\right), \\
\tilde{C}_{q q r, i_{1} S}^{(2) V S}\left({ }^{1} S_{0}\right)= & \tilde{C}_{r q q, i_{1} S}^{(1) V S}\left({ }^{1} S_{0}\right), \\
\tilde{C}_{r q q, i_{1} S}^{(3) V S}\left({ }^{1} S_{0}\right)= & -\frac{\beta^{2}}{4}+\frac{3}{4}(\widehat{m}-\widehat{m})-(\widehat{m}-\widehat{m}+3) \frac{\Delta_{A B}}{4} \\
& +\frac{\Delta_{A B}^{2}}{4}+\frac{\widehat{m}_{i_{1}}}{2}\left(3-\Delta_{A B}\right), \\
\tilde{C}_{q q r, i_{1} S}^{(4) V S}\left({ }^{1} S_{0}\right)= & \tilde{C}_{r q q, i_{1} S}^{(3) V S}\left({ }^{1} S_{0}\right) .
\end{aligned}
$$

There are additional non-vanishing kinematic factors for $\tilde{C}_{n, i_{1} X}^{(\alpha) V S}$ with $X=V$ or $S$, related to the corresponding expressions in (87 94) in the following way:

$$
\begin{aligned}
& \tilde{C}_{q q r, i_{1} X}^{(1) V S}\left({ }^{2 s+1} S_{J}\right)=\tilde{C}_{r q q, i_{1} X}^{(2) V S}\left({ }^{2 s+1} S_{J}\right)=-\left.\tilde{C}_{r r r, i_{1} X}^{(1) V S}\left({ }^{2 s+1} S_{J}\right)\right|_{\widehat{m}_{i_{1}} \rightarrow-\widehat{m}_{i_{1}}}, \\
& \tilde{C}_{q q r, i_{1} X}^{(3) V S}\left({ }^{2 s+1} S_{J}\right)=\tilde{C}_{r q q, i_{1} X}^{(4) V S}\left({ }^{2 s+1} S_{J}\right)=\left.\tilde{C}_{r r r, i_{1} X}^{(3) V S}\left({ }^{2 s+1} S_{J}\right)\right|_{\widehat{m}_{i_{1}} \rightarrow-\widehat{m}_{i_{1}}}, \\
& \tilde{C}_{q r q, i_{1} X}^{(1) V S}\left({ }^{2 s+1} S_{J}\right)=\tilde{C}_{q r q, i_{1} X}^{(2) V S}\left({ }^{2 s+1} S_{J}\right)=-\left.\tilde{C}_{r q q, i_{1} X}^{(1) V S}\left({ }^{2 s+1} S_{J}\right)\right|_{\widehat{m}_{i_{1}} \rightarrow-\widehat{m}_{i_{1}}}, \\
& \tilde{C}_{q r q, i_{1} X}^{(3) V S}\left({ }^{2 s+1} S_{J}\right)=\tilde{C}_{q r q, i_{1} X}^{(4) V S}\left({ }^{2 s+1} S_{J}\right)=\left.\tilde{C}_{r q q, i_{1} X}^{(3) V S}\left({ }^{2 s+1} S_{J}\right)\right|_{\widehat{m}_{i_{1}} \rightarrow-\widehat{m}_{i_{1}}} .
\end{aligned}
$$

The non-vanishing kinematic factors for $X_{A} X_{B}=V S$ and the four box-topologies $\alpha$ are given by

$$
\begin{aligned}
\tilde{D}_{r q q r, i_{1} i_{2}}^{(1) V S}\left({ }^{1} S_{0}\right)= & \frac{1}{4} \beta^{2}+\frac{1}{4}(\widehat{m}-\widehat{\bar{m}})^{2}-\frac{1}{4} \Delta_{A B}^{2}+\widehat{m}_{i_{1}} \widehat{m}_{i_{2}} \\
& +\frac{1}{2} \widehat{m}_{i_{1}}\left(\widehat{m}-\widehat{m}-\Delta_{A B}\right)+\frac{1}{2} \widehat{m}_{i_{2}}\left(\widehat{m}-\widehat{m}+\Delta_{A B}\right) \\
\tilde{D}_{r q q r, i_{1} i_{2}}^{(2) V S}\left({ }^{1} S_{0}\right)= & \frac{1}{4} \beta^{2}-\frac{1}{4}\left(\widehat{m}-\widehat{\bar{m}}+\Delta_{A B}\right)^{2}-\widehat{m}_{i_{1}} \widehat{m}_{i_{2}} \\
& -\frac{1}{2}\left(\widehat{m}_{i_{1}}+\widehat{m}_{i_{2}}\right)\left(\widehat{m}-\widehat{m}+\Delta_{A B}\right) \\
\tilde{D}_{r q q r, i_{1} i_{2}}^{(3) V S}\left({ }^{1} S_{0}\right)= & \left.\tilde{D}_{r q q r, i_{1} i_{2}}^{(1) V S}\left({ }^{1} S_{0}\right)\right|_{A \leftrightarrow B} \\
\tilde{D}_{r q q r, i_{1} i_{2}}^{(4) V S}\left({ }^{1} S_{0}\right)= & \left.\tilde{D}_{r q q r, i_{1} i_{2}}^{(2) V S}\left({ }^{1} S_{0}\right)\right|_{A \leftrightarrow B} .
\end{aligned}
$$

In case of ${ }^{3} S_{1}$ partial waves we have

$$
\tilde{D}_{r r r r, i_{1} i_{2}}^{(1) V S}\left({ }^{3} S_{1}\right)=-\frac{1}{12} \beta^{2}-\frac{1}{4}(\widehat{m}-\widehat{\bar{m}})^{2}+\frac{1}{4} \Delta_{A B}^{2}+\widehat{m}_{i_{1}} \widehat{m}_{i_{2}}
$$




$$
\begin{aligned}
& -\frac{1}{2} \widehat{m}_{i_{1}}\left(\widehat{m}-\widehat{\bar{m}}-\Delta_{A B}\right)+\frac{1}{2} \widehat{m}_{i_{2}}\left(\widehat{m}-\widehat{\bar{m}}+\Delta_{A B}\right), \\
\tilde{D}_{r r r r, i_{1} i_{2}}^{(2) V S}\left({ }^{3} S_{1}\right)= & -\frac{1}{12} \beta^{2}+\frac{1}{4}\left(\widehat{m}-\widehat{\bar{m}}+\Delta_{A B}\right)^{2}+\widehat{m}_{i_{1}} \widehat{m}_{i_{2}} \\
& +\frac{1}{2}\left(\widehat{m}_{i_{1}}+\widehat{m}_{i_{1}}\right)\left(\widehat{m}-\widehat{\bar{m}}+\Delta_{A B}\right), \\
\tilde{D}_{r r r r, i_{1} i_{2}}^{(3) V S}\left({ }^{3} S_{1}\right)= & \left.\tilde{D}_{r r r r, i_{1} i_{2}}^{(1) V S}\left({ }^{3} S_{1}\right)\right|_{\widehat{m} \leftrightarrow \widehat{m}}, \\
\tilde{D}_{r r r r, i_{1} i_{2}}^{(4) V S}\left({ }^{3} S_{1}\right)= & \left.\tilde{D}_{r r r r, i_{1} i_{2}}^{(2) V S}\left({ }^{3} S_{1}\right)\right|_{\widehat{m} \leftrightarrow \widehat{m}}, \\
\tilde{D}_{r q q r, i_{1} i_{2}}^{(\alpha) V S}\left({ }^{3} S_{1}\right)= & \frac{(-1)^{\alpha}}{6} \beta^{2} .
\end{aligned}
$$

Relations for the remaining kinematic factors for both ${ }^{1} S_{0}$ and ${ }^{3} S_{1}$ partial wave reactions read in case of diagram-topologies $\alpha=1,2$ :

$$
\begin{aligned}
& \tilde{D}_{q q q q, i_{1} i_{2}}^{(\alpha) V S}\left({ }^{2 s+1} S_{J}\right)=\left.(-1)^{\alpha} \tilde{D}_{r r r r, i_{1} i_{2}}^{(\alpha) V S}\left({ }^{2 s+1} S_{J}\right)\right|_{\hat{m}_{i_{1,2}} \rightarrow-\widehat{m}_{i_{1,2}},}, \\
& \tilde{D}_{r r q q, i_{1} i_{2}}^{(\alpha) V S}\left({ }^{2 s+1} S_{J}\right)=\left.(-1)^{\alpha+1} \tilde{D}_{r r r r, i_{1} i_{2}}^{(\alpha) V S}\left({ }^{2 s+1} S_{J}\right)\right|_{\hat{m}_{i_{2}} \rightarrow-\hat{m}_{i_{2}},}, \\
& \tilde{D}_{q q r r, i_{1} i_{2}}^{(\alpha) V S}\left({ }^{2 s+1} S_{J}\right)=-\left.\tilde{D}_{r r r r, i_{1} i_{2}}^{(\alpha) V S}\left({ }^{2 s+1} S_{J}\right)\right|_{\hat{m}_{i_{1}} \rightarrow-\hat{m}_{i_{1}},}, \\
& \tilde{D}_{q r r q, i_{1} i_{2}}^{(\alpha) V S}\left({ }^{(\alpha+1} S_{J}\right)=\left.(-1)^{\alpha} \tilde{D}_{r q q r, i_{1} i_{2}}^{(\alpha) V S}\left({ }^{2 s+1} S_{J}\right)\right|_{\hat{m}_{i_{1,2}} \rightarrow-\hat{m}_{i_{1,2}}}, \\
& \tilde{D}_{r q r q, i_{1} i_{2}}^{(\alpha) V S}\left({ }^{2 s+1} S_{J}\right)=\left.(-1)^{\alpha+1} \tilde{D}_{r q q r, i_{1} i_{2}}^{(\alpha) V S}\left({ }^{2 s+1} S_{J}\right)\right|_{\hat{m}_{i_{2}} \rightarrow-\hat{m}_{i_{2}}}, \\
& \tilde{D}_{q r q r, i_{1} i_{2}}^{(\alpha) V S}\left({ }^{2 s+1} S_{J}\right)=-\left.\tilde{D}_{r q q r, i_{1} i_{2}}^{(\alpha) V S}\left({ }^{2 s+1} S_{J}\right)\right|_{\hat{m}_{i_{1}} \rightarrow-\hat{m}_{i_{1}} .} .
\end{aligned}
$$

The corresponding relations for diagram-topologies $\alpha=3,4$ are given by

$$
\begin{aligned}
& \tilde{D}_{q q q q, i_{1} i_{2}}^{(\alpha) V S}\left({ }^{2 s+1} S_{J}\right)=\left.(-1)^{\alpha} \tilde{D}_{r r r r, i_{1} i_{2}}^{(\alpha) V S}\left({ }^{2 s+1} S_{J}\right)\right|_{\hat{m}_{i_{1,2}} \rightarrow-\widehat{m}_{i_{1,2}},}, \\
& \tilde{D}_{r r q q, i_{1} i_{2}}^{(\alpha) V S}\left({ }^{2 s+1} S_{J}\right)=\left.(-1)^{\alpha} \tilde{D}_{r r r r, i_{1} i_{2}}^{(\alpha) V S}\left({ }^{2 s+1} S_{J}\right)\right|_{\hat{m}_{i_{2}} \rightarrow-\hat{m}_{i_{2}},}, \\
& \tilde{D}_{q q r r, i_{1} i_{2}}^{(\alpha) V S}\left({ }^{2 s+1} S_{J}\right)=\left.\tilde{D}_{r r r r, i_{1} i_{2}}^{(\alpha) V S}\left({ }^{2 s+1} S_{J}\right)\right|_{\hat{m}_{i_{1}} \rightarrow-\hat{m}_{i_{1}},}, \\
& \tilde{D}_{q r r q, i_{1} i_{2}}^{(\alpha) V S}\left({ }^{2 s+1} S_{J}\right)=\left.(-1)^{\alpha} \tilde{D}_{r q q r, i_{1} i_{2}}^{(\alpha) V S}\left({ }^{2 s+1} S_{J}\right)\right|_{\hat{m}_{i_{1,2}} \rightarrow-\hat{m}_{i_{1,2}},}, \\
& \tilde{D}_{r q r q, i_{1} i_{2}}^{(\alpha) V S}\left({ }^{2 s+1} S_{J}\right)=\left.(-1)^{\alpha} \tilde{D}_{r q q r, i_{1} i_{2}}^{(\alpha) V S}\left({ }^{2 s+1} S_{J}\right)\right|_{\hat{m}_{i_{2}} \rightarrow-\hat{m}_{i_{2}},}, \\
& \tilde{D}_{q r q r, i_{1} i_{2}}^{(\alpha) V S}\left({ }^{2 s+1} S_{J}\right)=\left.\tilde{D}_{r q q r, i_{1} i_{2}}^{(\alpha) V S}\left({ }^{2 s+1} S_{J}\right)\right|_{\hat{m}_{i_{1}} \rightarrow-\hat{m}_{i_{1}} .},
\end{aligned}
$$

\section{A.3.3 Kinematic factors for $X_{A} X_{B}=S S$}

The non-vanishing $\tilde{B}_{n, i_{1} i_{2}}^{S S}$ terms with $i_{1} i_{2}=V V, V S, S V, V V$ read

$$
\tilde{B}_{q q, V V}^{S S}\left({ }^{1} S_{0}\right)=\Delta_{A B}^{2},
$$




$$
\begin{aligned}
\tilde{B}_{q q, V S}^{S S}\left({ }^{1} S_{0}\right) & =\tilde{B}_{q q, S V}^{S S}\left({ }^{1} S_{0}\right)=-\widehat{m}_{W} \Delta_{A B} \\
\tilde{B}_{q q, S S}^{S S}\left({ }^{1} S_{0}\right) & =\widehat{m}_{W}^{2}
\end{aligned}
$$

and in case of ${ }^{3} S_{1}$ reactions

$$
\tilde{B}_{r r, V V}^{S S}\left({ }^{3} S_{1}\right)=\frac{\beta^{2}}{3} .
$$

As in the case of $X_{A} X_{B}=V V$, the kinematic factors for $X_{A} X_{B}=S S$ and diagram topologies $\alpha=3,4$ are related to the corresponding expressions that arise from diagramtopologies $\alpha=1,2$. This applies to both triangle- and box-topologies (see Fig. [6] and Fig. 71):

$$
\begin{aligned}
& \tilde{C}_{n, i_{1} V}^{(3) S S}\left({ }^{2 s+1} S_{J}\right)=-\left.\tilde{C}_{n, i_{1} V}^{(1) S S}\left({ }^{2 s+1} S_{J}\right)\right|_{A \leftrightarrow B}, \\
& \tilde{C}_{n, i_{1} V}^{(4) S S}\left({ }^{2 s+1} S_{J}\right)=-\left.\tilde{C}_{n, i_{1} V}^{(2) S S}\left({ }^{2 s+1} S_{J}\right)\right|_{A \leftrightarrow B}, \\
& \tilde{C}_{n, i_{1} S}^{(3) S S}\left({ }^{2 s+1} S_{J}\right)=\left.\tilde{C}_{n, i_{1} S}^{(1) S S}\left({ }^{2 s+1} S_{J}\right)\right|_{A \leftrightarrow B}, \\
& \tilde{C}_{n, i_{1} S}^{(4) S S}\left({ }^{2 s+1} S_{J}\right)=\left.\tilde{C}_{n, i_{1} S}^{(2) S S}\left({ }^{2 s+1} S_{J}\right)\right|_{A \leftrightarrow B}, \\
& \tilde{D}_{n, i_{1} i_{2}}^{(3) S S}\left({ }^{2 s+1} S_{J}\right)=\left.\tilde{D}_{n, i_{1} i_{2}}^{(1) S S}\left({ }^{2 s+1} S_{J}\right)\right|_{A \leftrightarrow B}, \\
& \tilde{D}_{n, i_{1} i_{2}}^{(4) S S}\left({ }^{2 s+1} S_{J}\right)=\left.\tilde{D}_{n, i_{1} i_{2}}^{(2) S S}\left({ }^{2 s+1} S_{J}\right)\right|_{A \leftrightarrow B} .
\end{aligned}
$$

In case of expressions $\tilde{C}_{n, i_{1} V}^{(\alpha) S S}$ for diagram-topologies $\alpha=1,2$ we find

$$
\begin{aligned}
& \tilde{C}_{r q q, i_{1} V}^{(1) S S}\left({ }^{1} S_{0}\right)=\tilde{C}_{q q r, i_{1} V}^{(2) S S}\left({ }^{1} S_{0}\right)=\frac{\Delta_{A B}}{2}\left(\widehat{m}-\widehat{\bar{m}}+\Delta_{A B}\right)+\widehat{m}_{i_{1}} \Delta_{A B}, \\
& \tilde{C}_{r r r, i_{1} V}^{(1) S S}\left({ }^{3} S_{1}\right)=\tilde{C}_{r r r, i_{1} V}^{(2) S S}\left({ }^{3} S_{1}\right)=-\frac{\beta^{2}}{6} .
\end{aligned}
$$

The $\tilde{C}_{n, i_{1} S}^{(\alpha) S S}$ expressions with $\alpha=1,2$ are given by

$$
\tilde{C}_{r q q, i_{1} S}^{(1) S S}\left({ }^{1} S_{0}\right)=\tilde{C}_{q q r, i_{1} S}^{(2) S S}\left({ }^{1} S_{0}\right)=-\frac{\widehat{m}_{W}}{2}\left(\widehat{m}-\widehat{\bar{m}}+\Delta_{A B}\right)-\widehat{m}_{W} \widehat{m}_{i_{1}} .
$$

All other non-vanishing expressions for $\tilde{C}_{n, i_{1} X}^{(\alpha) S S}$ with $X=V, S$ and $\alpha=1,2$ can be related to the terms in (112 114) in the following way:

$$
\begin{aligned}
& \tilde{C}_{q q r, i_{1} X}^{(1) S S}\left({ }^{2 s+1} S_{J}\right)=\tilde{C}_{r q q, i_{1} X}^{(2) S S}\left({ }^{2 s+1} S_{J}\right)=-\left.\tilde{C}_{r r r, i_{1} X}^{(1) S S}\left({ }^{2 s+1} S_{J}\right)\right|_{\widehat{m}_{i_{1}} \rightarrow-\widehat{m}_{i_{1}}}, \\
& \tilde{C}_{q r q, i_{1} X}^{(1) S S}\left({ }^{2 s+1} S_{J}\right)=\tilde{C}_{q r q, i_{1} X}^{(2) S S}\left({ }^{2 s+1} S_{J}\right)=-\left.\tilde{C}_{r q q, i_{1} X}^{(1) S S}\left({ }^{2 s+1} S_{J}\right)\right|_{\widehat{m}_{i_{1}} \rightarrow-\widehat{m}_{i_{1}}} .
\end{aligned}
$$

The expressions $\tilde{D}_{n, i_{1} i_{2}}^{(\alpha) S S}$ for diagram-topologies $\alpha=1,2$ and ${ }^{1} S_{0}$ partial waves read

$$
\tilde{D}_{r q q r, i_{1} i_{2}}^{\left.(1) S{ }^{1} S_{0}\right)}=\frac{1}{4}(\widehat{m}-\widehat{\bar{m}})^{2}-\frac{\Delta_{A B}^{2}}{4}+\widehat{m}_{i_{1}} \widehat{m}_{i_{2}}
$$




$$
\begin{aligned}
& +\frac{\widehat{m}_{i_{1}}}{2}\left(\widehat{m}-\widehat{\bar{m}}-\Delta_{A B}\right)+\frac{\widehat{m}_{i_{2}}}{2}\left(\widehat{m}-\widehat{\bar{m}}+\Delta_{A B}\right), \\
\tilde{D}_{r q q r, i_{1} i_{2}}^{(2) S S}\left({ }^{1} S_{0}\right)= & \frac{1}{4}\left(\widehat{m}-\widehat{\bar{m}}+\Delta_{A B}\right)^{2}+\widehat{m}_{i_{1}} \widehat{m}_{i_{2}} \\
& +\frac{1}{2}\left(\widehat{m}_{i_{1}}+\widehat{m}_{i_{2}}\right)\left(\widehat{m}-\widehat{\bar{m}}+\Delta_{A B}\right) .
\end{aligned}
$$

In case of a ${ }^{3} S_{1}$ partial wave configuration we find

$$
\tilde{D}_{r r r r, i_{1} i_{2}}^{(\alpha) S S}\left({ }^{3} S_{1}\right)=(-1)^{\alpha} \frac{\beta^{2}}{12}
$$

The remaining non-vanishing kinematic factors related to both ${ }^{1} S_{0}$ and ${ }^{3} S_{1}$ partial-wave reactions read

$$
\begin{aligned}
& \tilde{D}_{q q q q, i_{1} i_{2}}^{(\alpha) S S}\left({ }^{2 s+1} S_{J}\right)=\tilde{D}_{r r r r, i_{1} i_{2}}^{(\alpha) S S}\left({ }^{2 s+1} S_{J}\right) \\
& \tilde{D}_{r r q q, i_{1} i_{2}}^{(\alpha) S S}\left({ }^{2 s+1} S_{J}\right)=\tilde{D}_{q q r r, i_{1} i_{2}}^{(\alpha) S S}\left({ }^{2 s+1} S_{J}\right)=-\tilde{D}_{r r r r, i_{1} i_{2}}^{(\alpha) S S}\left({ }^{2 s+1} S_{J}\right), \\
& \tilde{D}_{q r r q, i_{1} i_{2}}^{(\alpha) S S}\left({ }^{2 s+1} S_{J}\right)=\left.\tilde{D}_{r q q r, i_{1} i_{2}}^{(\alpha) S S}\left({ }^{2 s+1} S_{J}\right)\right|_{\hat{m}_{i_{1,2}} \rightarrow-\hat{m}_{i_{1,2}}}, \\
& \tilde{D}_{r q r q, i_{1} i_{2}}^{(\alpha) S S}\left({ }^{2 s+1} S_{J}\right)=-\left.\tilde{D}_{r q q r, i_{1} i_{2}}^{(\alpha) S S}\left({ }^{2 s+1} S_{J}\right)\right|_{\hat{m}_{i_{2}} \rightarrow-\hat{m}_{i_{2}}}, \\
& \tilde{D}_{q r q r, i_{1} i_{2}}^{(\alpha) S S}\left({ }^{2 s+1} S_{J}\right)=-\left.\tilde{D}_{r q q r, i_{1} i_{2}}^{(\alpha) S S}\left({ }^{2 s+1} S_{J}\right)\right|_{\hat{m}_{i_{1}} \rightarrow-\hat{m}_{i_{1}}} .
\end{aligned}
$$

\section{A.3.4 Kinematic factors for $X_{A} X_{B}=f f$}

The non-vanishing $\tilde{B}_{n, i_{1} i_{2}}^{f f}$ terms with $i_{1} i_{2}=V V, V S, S V, S S$ are given by

$$
\begin{aligned}
& \tilde{B}_{q q q q, V V}^{f f}\left({ }^{1} S_{0}\right)=1-\beta^{2}+4 \widehat{m}_{A} \widehat{m}_{B}-\Delta_{A B}^{2}, \\
& \tilde{B}_{q q q q, V S}^{f f}\left({ }^{1} S_{0}\right)=\tilde{B}_{q q q q, S V}^{f f}\left({ }^{1} S_{0}\right)=2\left(\widehat{m}_{A}+\widehat{m}_{B}-\left(\widehat{m}_{A}-\widehat{m}_{B}\right) \Delta_{A B}\right), \\
& \tilde{B}_{q q q q, S S}^{f f}\left({ }^{1} S_{0}\right)=1+\beta^{2}+4 \widehat{m}_{A} \widehat{m}_{B}-\Delta_{A B}^{2},
\end{aligned}
$$

and in case of ${ }^{3} S_{1}$ partial-wave reactions

$$
\tilde{B}_{r r r r, V V}^{f f}\left({ }^{3} S_{1}\right)=1+\frac{\beta^{2}}{3}+4 \widehat{m}_{A} \widehat{m}_{B}-\Delta_{A B}^{2}
$$

There are additional non-vanishing terms $\tilde{B}_{n, i_{1} i_{2}}^{f f}$ related to the expressions in (120 123). In case of $i_{1} i_{2}=V V, S S$, the corresponding relations read

$$
\begin{aligned}
& \tilde{B}_{r q q r, i_{1} i_{2}}^{f f}\left({ }^{2 s+1} S_{J}\right)=\left.\tilde{B}_{r r r r, i_{1} i_{2}}^{f f}\left({ }^{2 s+1} S_{J}\right)\right|_{\widehat{m}_{A} \widehat{m}_{B} \rightarrow-\widehat{m}_{A} \widehat{m}_{B}}, \\
& \tilde{B}_{q r r q, i_{1} i_{2}}^{f f}\left({ }^{2 s+1} S_{J}\right)=\left.\tilde{B}_{q q q q, i_{1} i_{2}}^{f f}\left({ }^{2 s+1} S_{J}\right)\right|_{\widehat{m}_{A} \widehat{m}_{B} \rightarrow-\widehat{m}_{A} \widehat{m}_{B}},
\end{aligned}
$$


and our notation implies, that the product $\widehat{m}_{A} \widehat{m}_{B}$ has to be replaced, but all other occurrences of $\widehat{m}_{A}$ or $\widehat{m}_{B}$ are untouched. Similarly, in case of $i_{1} i_{2}=V S, S V$, the additional non-vanishing $\tilde{B}_{n, i_{1} i_{2}}^{f f}$ terms are given by

$$
\begin{aligned}
& \tilde{B}_{r q q r, i_{1} i_{2}}^{f f}\left({ }^{2 s+1} S_{J}\right)=-\left.\tilde{B}_{r r r r, i_{1} i_{2}}^{f f}\left({ }^{2 s+1} S_{J}\right)\right|_{\widehat{m}_{A} \rightarrow-\widehat{m}_{A}}, \\
& \tilde{B}_{q r r q, i_{1} i_{2}}^{f f}\left({ }^{2 s+1} S_{J}\right)=-\left.\tilde{B}_{q q q q, i_{1} i_{2}}^{f f}\left({ }^{2 s+1} S_{J}\right)\right|_{\widehat{m}_{A} \rightarrow-\widehat{m}_{A}} .
\end{aligned}
$$

The relations among kinematic factors for diagram topologies $\alpha=3,4$ and diagramtopologies $\alpha=1,2$ in both the cases of box- and triangle-topologies are given by $(X=$ $V, S)$

$$
\begin{aligned}
& C_{n, i_{1} X}^{(3) f f}\left({ }^{2 s+1} S_{J}\right)=\left.C_{n, i_{1} X}^{(1) f f}\left({ }^{2 s+1} S_{J}\right)\right|_{A \leftrightarrow B}, \\
& C_{n, i_{1} X}^{(4) f f}\left({ }^{2 s+1} S_{J}\right)=\left.C_{n, i_{1} X}^{(2) f f}\left({ }^{2 s+1} S_{J}\right)\right|_{A \leftrightarrow B}, \\
& D_{n, i_{1} i_{2}}^{(3) f f}\left({ }^{2 s+1} S_{J}\right)=\left.D_{n, i_{1} i_{2}}^{(1) f f}\left({ }^{2 s+1} S_{J}\right)\right|_{A \leftrightarrow B}, \\
& D_{n, i_{1} i_{2}}^{(4) f f}\left({ }^{2 s+1} S_{J}\right)=\left.D_{n, i_{1} i_{2}}^{(2) f f}\left({ }^{2 s+1} S_{J}\right)\right|_{A \leftrightarrow B},
\end{aligned}
$$

compare to the generic diagrams in Fig. 6] and Fig. 8. The structures $\tilde{C}_{n, i_{1} V}^{(\alpha) f f}$ for topologies $\alpha=1,2$ are given by

$$
\begin{aligned}
\tilde{C}_{q q q q, i_{1} V}^{(\alpha) f f}\left({ }^{1} S_{0}\right)= & \frac{\beta^{2}}{4}-\frac{1}{4}\left(1-2 \widehat{m}_{A}\right)\left(1-2 \widehat{m}_{B}\right) \\
& -\left(\widehat{m}_{A}-\widehat{m}_{B}\right) \frac{\Delta_{A B}}{2}-\frac{\Delta_{A B}^{2}}{4},
\end{aligned}
$$

and in case of ${ }^{3} S_{1}$ partial wave reactions the respective expressions read

$$
\begin{aligned}
\tilde{C}_{r r r r, i_{1} V}^{(\alpha) f f}\left({ }^{3} S_{1}\right)= & -\frac{\beta^{2}}{12}-\frac{1}{4}\left(1+2 \widehat{m}_{A}\right)\left(1+2 \widehat{m}_{B}\right) \\
& +\left(\widehat{m}_{A}-\widehat{m}_{B}\right) \frac{\Delta_{A B}}{2}+\frac{\Delta_{A B}^{2}}{4}
\end{aligned}
$$

The relations of the additional non-vanishing $\tilde{C}_{n, i_{1} V}^{(\alpha) f f}$ expressions to the respective terms in (132 133) read

$$
\begin{aligned}
& \tilde{C}_{q q r r, i_{1} V}^{(1) f f}\left({ }^{2 s+1} S_{J}\right)=\tilde{C}_{r r q q, i_{1} V}^{(2) f f}\left({ }^{2 s+1} S_{J}\right)=-\left.\tilde{C}_{r r r r, i_{1} V}^{(1) f f}\left({ }^{2 s+1} S_{J}\right)\right|_{m_{A, B} \rightarrow-m_{A}, B}, \\
& \tilde{C}_{r q q r, i_{1} V}^{(\alpha) f f}\left({ }^{2 s+1} S_{J}\right)=\left.\tilde{C}_{r r r r, i_{1} V}^{(\alpha) f f}\left({ }^{2 s+1} S_{J}\right)\right|_{m_{A} \rightarrow-m_{A}}, \\
& \tilde{C}_{q r q r, i_{1} V}^{(1) f f}\left({ }^{2 s+1} S_{J}\right)=\tilde{C}_{r q r q, i_{1} V}^{(2) f f}\left({ }^{2 s+1} S_{J}\right)=-\left.\tilde{C}_{r r r r, i_{1} V}^{(1) f f}\left({ }^{2 s+1} S_{J}\right)\right|_{m_{B} \rightarrow-m_{B}}, \\
& \tilde{C}_{r q r q, i_{1} V}^{(1) f f}\left({ }^{2 s+1} S_{J}\right)=\tilde{C}_{q r q r, i_{1} V}^{(2) f f}\left({ }^{2 s+1} S_{J}\right)=-\left.\tilde{C}_{q q q q, i_{1} V}^{(\alpha) f f}\left({ }^{2 s+1} S_{J}\right)\right|_{m_{B} \rightarrow-m_{B}},
\end{aligned}
$$




$$
\begin{aligned}
& \tilde{C}_{q r r q, i_{1} V}^{(\alpha) f f}\left({ }^{2 s+1} S_{J}\right)=\left.\tilde{C}_{q q q q, i_{1} V}^{(\alpha) f f}\left({ }^{2 s+1} S_{J}\right)\right|_{m_{A} \rightarrow-m_{A}}, \\
& \tilde{C}_{r r q q, i_{1} V}^{(1) f f}\left({ }^{2 s+1} S_{J}\right)=\tilde{C}_{q q r r, i_{1} V}^{(2) f f}\left({ }^{2 s+1} S_{J}\right)=-\left.\tilde{C}_{q q q q, i_{1} V}^{(\alpha) f f}\left({ }^{2 s+1} S_{J}\right)\right|_{m_{A, B} \rightarrow-m_{A, B}} .
\end{aligned}
$$

The terms $\tilde{C}_{n, i_{1} S}^{(\alpha) f f}$ for $\alpha=1,2 \operatorname{read}$

$$
\begin{aligned}
\tilde{C}_{q q q q, i_{1} S}^{(\alpha) f f}\left({ }^{1} S_{0}\right)= & \frac{\beta^{2}}{4}+\frac{1}{4}\left(1-2 \widehat{m}_{A}\right)\left(1-2 \widehat{m}_{B}\right) \\
& +\left(\widehat{m}_{A}-\widehat{m}_{B}\right) \frac{\Delta_{A B}}{2}-\frac{\Delta_{A B}^{2}}{4}
\end{aligned}
$$

and all remaining non-vanishing $C_{n, i_{1} S}^{(\alpha) f f}$ terms are obtained from (135) in the following way:

$$
\begin{aligned}
& \tilde{C}_{r q r q, i_{1} S}^{(1) f f}\left({ }^{2 s+1} S_{J}\right)=\tilde{C}_{q r q r, i_{1} S}^{(2) f f}\left({ }^{2 s+1} S_{J}\right)=\left.\tilde{C}_{q q q q, i_{1} S}^{(\alpha) f f}\left({ }^{2 s+1} S_{J}\right)\right|_{m_{B} \rightarrow-m_{B}}, \\
& \tilde{C}_{q r r q, i_{1} S}^{(\alpha) f f}\left({ }^{2 s+1} S_{J}\right)=\left.\tilde{C}_{q q q q, i_{1} S}^{(\alpha) f f}\left({ }^{2 s+1} S_{J}\right)\right|_{m_{A} \rightarrow-m_{A}}, \\
& \tilde{C}_{r r q q, i_{1} S}^{(1) f f}\left({ }^{2 s+1} S_{J}\right)=\tilde{C}_{q q r r, i_{1} S}^{(2) f f}\left({ }^{2 s+1} S_{J}\right)=\left.\tilde{C}_{q q q q, i_{1} S}^{(\alpha) f f}\left({ }^{2 s+1} S_{J}\right)\right|_{m_{A, B} \rightarrow-m_{A, B}} .
\end{aligned}
$$

In case of box-diagram topologies $\alpha=1,2$, we find the following $\tilde{D}_{n, i_{1} i_{2}}^{(\alpha) f f}$ structures for the ${ }^{1} S_{0}$ partial waves:

$$
\begin{aligned}
& \tilde{D}_{r r r r, i_{1} i_{2}}^{(\alpha) f f}\left({ }^{1} S_{0}\right)=\frac{1}{8}\left(1+2 \widehat{m}_{B}-\Delta_{A B}\right)\left(1+2 \widehat{m}_{A}+\Delta_{A B}\right), \\
& \tilde{D}_{r r q q, i_{1} i_{2}}^{(\alpha) f f}\left({ }^{1} S_{0}\right)=\frac{\beta^{2}}{8}-\frac{1}{2} \widehat{m}_{A} \widehat{m}_{B} .
\end{aligned}
$$

For ${ }^{3} S_{1}$ partial-wave configurations we have

$$
\begin{aligned}
& \tilde{D}_{r r r r i_{1} i_{2}}^{(\alpha) f f}\left({ }^{3} S_{1}\right)=(-1)^{\alpha} \tilde{D}_{r r r r, i_{1} i_{2}}^{(\alpha) f f}\left({ }^{1} S_{0}\right), \\
& \tilde{D}_{r r q q, i_{1} i_{2}}^{(\alpha) f f}\left({ }^{3} S_{1}\right)=(-1)^{\alpha+1}\left(\frac{\beta^{2}}{24}+\frac{1}{2} \widehat{m}_{A} \widehat{m}_{B}\right) .
\end{aligned}
$$

Relations for the remaining non-vanishing kinematic factors related to both ${ }^{1} S_{0}$ and ${ }^{3} S_{1}$ partial-wave processes read in case of diagram topology $\alpha=1$

$$
\begin{aligned}
\tilde{D}_{q q q q, i_{1} i_{2}}^{(1) f f}\left({ }^{2 s+1} S_{J}\right) & =\left.\tilde{D}_{r r r r, i_{1} i_{2}}^{(1) f f}\left({ }^{2 s+1} S_{J}\right)\right|_{\hat{m}_{A, B} \rightarrow-\hat{m}_{A, B}}, \\
\tilde{D}_{q q r r, i_{1} i_{2}}^{(1) f f}\left({ }^{2 s+1} S_{J}\right) & =\tilde{D}_{r r q q, i_{1} i_{2}}^{(1) f f}\left({ }^{2 s+1} S_{J}\right), \\
\tilde{D}_{r q q r, i_{1} i_{2}}^{(1) f f}\left({ }^{2 s+1} S_{J}\right) & =\tilde{D}_{q r r q, i_{1} i_{2}}^{(1) f f}\left({ }^{2 s+1} S_{J}\right)=\left.\tilde{D}_{r r q q, i_{1} i_{2}}^{(1) f f}\left({ }^{2 s+1} S_{J}\right)\right|_{\hat{m}_{A} \hat{m}_{B} \rightarrow-\hat{m}_{A} \hat{m}_{B}}, \\
\tilde{D}_{r q r q, i_{1} i_{2}}^{(1) f f}\left({ }^{2 s+1} S_{J}\right) & =\left.\tilde{D}_{r r r r, i_{1} i_{2}}^{(1) f f}\left({ }^{2 s+1} S_{J}\right)\right|_{\hat{m}_{A} \rightarrow-\hat{m}_{A}}, \\
\tilde{D}_{q r q r, i_{1} i_{2}}^{(1) f f}\left({ }^{2 s+1} S_{J}\right) & =\left.\tilde{D}_{r r r r, i_{1} i_{2}}^{(1) f f}\left({ }^{2 s+1} S_{J}\right)\right|_{\hat{m}_{B} \rightarrow-\hat{m}_{B}} .
\end{aligned}
$$


In case of diagram topology $\alpha=2$, the corresponding relations are given by

$$
\begin{aligned}
\tilde{D}_{q q q q, i_{1} i_{2}}^{(2) f f}\left({ }^{2 s+1} S_{J}\right) & =\left.\tilde{D}_{r r r r, i_{1} i_{2}}^{(2) f f}\left({ }^{2 s+1} S_{J}\right)\right|_{\hat{m}_{A, B} \rightarrow-\hat{m}_{A, B}}, \\
\tilde{D}_{q q r r, i_{1} i_{2}}^{(2) f f}\left({ }^{2 s+1} S_{J}\right) & =\tilde{D}_{r r q q, i_{1} i_{2}}^{(2) f f}\left({ }^{2 s+1} S_{J}\right), \\
\tilde{D}_{r q q r, i_{1} i_{2}}^{(2) f f}\left({ }^{2 s+1} S_{J}\right) & =\left.\tilde{D}_{r r r r, i_{1} i_{2}}^{(2) f f}\left({ }^{2 s+1} S_{J}\right)\right|_{\hat{m}_{A} \rightarrow-\hat{m}_{A}}, \\
\tilde{D}_{q r r q, i_{1} i_{2}}^{(2) f f}\left({ }^{2 s+1} S_{J}\right) & =\left.\tilde{D}_{r r r r, i_{1} i_{2}}^{(2) f f}\left({ }^{2 s+1} S_{J}\right)\right|_{\hat{m}_{B} \rightarrow-\hat{m}_{B}}, \\
\tilde{D}_{r q r q, i_{1} i_{2}}^{(2) f f}\left({ }^{2 s+1} S_{J}\right) & =\tilde{D}_{q r q r, i_{1} i_{2}}^{(2) f f}\left({ }^{2 s+1} S_{J}\right)=\left.\tilde{D}_{r r q q, i_{1} i_{2}}^{(2) f f}\left({ }^{2 s+1} S_{J}\right)\right|_{\hat{m}_{A} \hat{m}_{B} \rightarrow-\hat{m}_{A} \hat{m}_{B}} .
\end{aligned}
$$

\section{A.3.5 Kinematic factors for $X_{A} X_{B}=\eta \bar{\eta}$}

In case of $X_{A} X_{B}=\eta \bar{\eta}$ one cannot directly construct the coupling factors $b_{n} i_{1} i_{2}$ using the recipe given in Sec. A.2, which is based on considering the $\chi_{e_{1}} \chi_{e_{2}} \rightarrow X_{A} X_{B}$ and $\chi_{e_{4}} \chi_{e_{3}} \rightarrow X_{A} X_{B}$ tree-level annihilation amplitudes. In order to obtain the coupling factor expressions $b_{n i_{1} i_{2}}$, that correspond to the kinematic factors presented below, one should proceed as follows: First extract the (axial-) vector and (pseudo-) scalar coupling factors associated with the interaction of the $\chi_{e_{1}} \chi_{e_{2}}$ or $\chi_{e_{4}} \chi_{e_{3}}$ pair and the $s$-channel exchanged particle species. This is done following the steps 1 . and 2 . in the recipe given in Sec. A.2. Next, complex-conjugate the couplings related to the $\chi_{e_{4}} \chi_{e_{3}}$ particle pair. In order to determine the couplings to the ghosts, consider the 1-loop amplitude $\chi_{e_{1}} \chi_{e_{2}} \rightarrow \eta \bar{\eta} \rightarrow \chi_{e_{4}} \chi_{e_{3}}$, similar to the selfenergy-amplitude in Fig. 5. Assign a ghost flow to the lower line of the 1-loop amplitude (labelled with $X_{A}$ in Fig. 5), that flows from left to right. Consequently there is a ghost flow from right to left on the upper line, which is labelled with $X_{B}$. Assume that the coupling factors at each of the two ghost vertices are generically of the form $i g_{2} c_{A B X_{i}} L_{A B X_{i}}$, where the Lorentz structures $L_{A B X_{i}}$ are defined in Tab. 4. Determine the expressions that replace the generic $c_{A B X_{i}}$ factors for the specific process under consideration. Now build all possible combinations of two-coupling factor products from the set of the neutralino/chargino couplings to the $s$-channel exchanged particles (including factors of -1 in front of vector couplings) in the $\chi_{e_{1}} \chi_{e_{2}} \rightarrow \eta \bar{\eta} \rightarrow \chi_{e_{4}} \chi_{e_{3}}$ reaction, and multiply them by the $c_{A B X_{i_{1}}}$ and $c_{A B X_{i_{2}}}$ factors. The convention for the naming of the resulting coupling factor expressions $b_{n, i_{1} i_{2}}$ with subscripts $n=r r, q q$ is the same as in the cases $X_{A} X_{B}=V V, V S, S S$, see Sec. A.2. The coupling factors $b_{n, i_{1} i_{2}}$ derived in this way correspond to the kinematic factors given below. Note that the mass parameter $m_{A}$ in the expressions below refers to the mass of the ghost flowing in the lower line, and $m_{B}$ to the mass of the ghost in the upper line.

The non-vanishing $\tilde{B}_{n, i_{1} i_{2}}^{\eta \bar{\eta}}$ terms with $i_{1} i_{2}=V V, V S, S V, S S$ read

$$
\begin{aligned}
\tilde{B}_{q q, V V}^{\eta \bar{\eta}}\left({ }^{1} S_{0}\right) & =\frac{1}{4}\left(1-\Delta_{A B}^{2}\right), \\
\tilde{B}_{q q, V S}^{\eta \bar{\eta}}\left({ }^{1} S_{0}\right) & =-\frac{\widehat{m}_{W}}{2}\left(1+\Delta_{A B}\right),
\end{aligned}
$$




$$
\begin{aligned}
\tilde{B}_{q,, S V}^{\eta \bar{\eta}}\left({ }^{1} S_{0}\right) & =\frac{\widehat{m}_{W}}{2}\left(1-\Delta_{A B}\right), \\
\tilde{B}_{q q, S S}^{\eta \bar{\eta}}\left({ }^{1} S_{0}\right) & =-\widehat{m}_{W}^{2} .
\end{aligned}
$$

Similarly,

$$
\tilde{B}_{r r, V V}^{\eta \bar{\eta}}\left({ }^{3} S_{1}\right)=-\frac{\beta^{2}}{12} .
$$

\section{References}

[1] Particle Data Group Collaboration, J. Beringer et al., Phys.Rev. D86 (2012) 010001

[2] G. Jungman, M. Kamionkowski, and K. Griest, Phys.Rept. 267 (1996) 195-373, arXiv:hep-ph/9506380 [hep-ph].

[3] G. Bertone, D. Hooper, and J. Silk, Phys.Rept. 405 (2005) 279-390, arXiv:hep-ph/0404175 [hep-ph].

[4] P. Gondolo, J. Edsjo, P. Ullio, L. Bergstrom, M. Schelke, et al., JCAP 0407 (2004) 008, arXiv: astro-ph/0406204 [astro-ph].

[5] G. Belanger, F. Boudjema, P. Brun, A. Pukhov, S. Rosier-Lees, et al., Comput.Phys.Commun. 182 (2011) 842-856, arXiv:1004.1092 [hep-ph].

[6] B. Herrmann and M. Klasen, Phys.Rev. D76 (2007) 117704,

arXiv:0709.0043 [hep-ph].

[7] B. Herrmann, M. Klasen, and K. Kovarik, Phys.Rev. D79 (2009) 061701, arXiv:0901.0481 [hep-ph].

[8] B. Herrmann, M. Klasen, and K. Kovarik, Phys.Rev. D80 (2009) 085025, arXiv:0907.0030 [hep-ph].

[9] F. Boudjema, G. Drieu La Rochelle, and S. Kulkarni, Phys.Rev. D84 (2011) 116001, arXiv:1108.4291 [hep-ph].

[10] A. Chatterjee, M. Drees, and S. Kulkarni, arXiv:1209.2328 [hep-ph].

[11] N. Baro, F. Boudjema, and A. Semenov, Phys.Lett. B660 (2008) 550-560, arXiv:0710.1821 [hep-ph].

[12] N. Baro, F. Boudjema, G. Chalons, and S. Hao, Phys.Rev. D81 (2010) 015005, arXiv:0910.3293 [hep-ph].

[13] J. Hisano, S. Matsumoto, M. M. Nojiri, and O. Saito, \begin{tabular}{|l}
\hline Phys.Rev. D71 (2005) 063528, arXiv:hep-ph/0412403 [hep-ph]. \\
\hline
\end{tabular} 
[14] J. Hisano, S. Matsumoto, M. Nagai, O. Saito, and M. Senami, Phys.Lett. B646 (2007) 34-38, arXiv:hep-ph/0610249 [hep-ph].

[15] M. Cirelli, A. Strumia, and M. Tamburini, Nucl.Phys. B787 (2007) 152-175, arXiv:0706.4071 [hep-ph].

[16] N. Arkani-Hamed, D. P. Finkbeiner, T. R. Slatyer, and N. Weiner, Phys.Rev. D79 (2009) 015014, arXiv:0810.0713 [hep-ph].

[17] M. Drees, J. Kim, and K. Nagao, Phys.Rev. D81 (2010) 105004. arXiv:0911.3795 [hep-ph].

[18] A. Hryczuk, R. Iengo, and P. Ullio, JHEP 1103 (2011) 069, arXiv:1010.2172 [hep-ph].

[19] A. Hryczuk, Phys.Lett. B699 (2011) 271-275, arXiv:1102.4295 [hep-ph].

[20] A. Hryczuk and R. Iengo, JHEP 1201 (2012) 163, arXiv:1111.2916 [hep-ph].

[21] G. T. Bodwin, E. Braaten, and G. P. Lepage, Phys.Rev. D51 (1995) 1125-1171, arXiv:hep-ph/9407339 [hep-ph].

[22] M. Drees and M. M. Nojiri, Phys.Rev. D47 (1993) 376-408, arXiv:hep-ph/9207234[hep-ph].

[23] C. Hellmann and P. Ruiz-Femenia, JHEP 1308 (2013) 084, arXiv:1303.0200 [hep-ph].

[24] M. Beneke, C. Hellmann, and P. Ruiz-Femenía. In preparation.

[25] A. Djouadi, J.-L. Kneur, and G. Moultaka, Comput.Phys.Commun. 176 (2007) 426-455, arXiv:hep-ph/0211331 [hep-ph].

[26] J. Alwall, M. Herquet, F. Maltoni, O. Mattelaer, and T. Stelzer, JHEP 1106 (2011) 128, arXiv:1106.0522 [hep-ph],

[27] P. Gondolo and G. Gelmini, Nucl.Phys. B360 (1991) 145-179.

[28] K. Griest and D. Seckel, Phys.Rev. D43 (1991) 3191-3203.

[29] N. Baro, F. Boudjema, and A. Semenov, Phys.Rev. D78 (2008) 115003, arXiv:0807.4668 [hep-ph].

[30] J. Vermaseren, Comput.Phys.Commun. 83 (1994) 45-58.

[31] D. Binosi, J. Collins, C. Kaufhold, and L. Theussl, Comput.Phys.Commun. 180 (2009) 1709-1715, arXiv:0811.4113 [hep-ph].

[32] A. Denner, H. Eck, O. Hahn, and J. Kublbeck, Nucl.Phys. B387 (1992) 467-484. 
[33] J. Rosiek, Phys.Rev. D41 (1990) 3464, arXiv:hep-ph/9511250 [hep-ph]. Erratum: ibid. vol. 387, p. 830 (1997).

[34] M. Beneke, C. Hellmann and P. Ruiz-Femenia, JHEP 1303 (2013) 148 [Erratum-ibid. 1310 (2013) 224] [arXiv:1210.7928 [hep-ph]]. 